\title{
Quadriceps muscle ultrasound as a new tool for diagnosing muscle wasting in renal diseases
}

Citation for published version (APA):

Baganha Sabatino, A. (2021). Quadriceps muscle ultrasound as a new tool for diagnosing muscle wasting in renal diseases. [Doctoral Thesis, Maastricht University]. Ridderprint. https://doi.org/10.26481/dis.20211213as

Document status and date:

Published: 01/01/2021

DOI:

10.26481/dis.20211213as

Document Version:

Publisher's PDF, also known as Version of record

\section{Please check the document version of this publication:}

- A submitted manuscript is the version of the article upon submission and before peer-review. There can be important differences between the submitted version and the official published version of record.

People interested in the research are advised to contact the author for the final version of the publication, or visit the DOI to the publisher's website.

- The final author version and the galley proof are versions of the publication after peer review.

- The final published version features the final layout of the paper including the volume, issue and page numbers.

Link to publication

\footnotetext{
General rights rights.

- You may freely distribute the URL identifying the publication in the public portal. please follow below link for the End User Agreement:

www.umlib.nl/taverne-license

Take down policy

If you believe that this document breaches copyright please contact us at:

repository@maastrichtuniversity.nl

providing details and we will investigate your claim.
}

Copyright and moral rights for the publications made accessible in the public portal are retained by the authors and/or other copyright owners and it is a condition of accessing publications that users recognise and abide by the legal requirements associated with these

- Users may download and print one copy of any publication from the public portal for the purpose of private study or research.

- You may not further distribute the material or use it for any profit-making activity or commercial gain

If the publication is distributed under the terms of Article $25 \mathrm{fa}$ of the Dutch Copyright Act, indicated by the "Taverne" license above, 
QUADRICEPS MUSCLE ULTRASOUND AS A NEW TOOL FOR

DIAGNOSING MUSCLE WASTING IN RENAL DISEASES

Alice Sabatino

2021 
The research presented in this thesis was conducted at the Nephrology and Dialysis unit of the Parma University Hospital, Parma, Italy. This study was performed in scientific collaboration with the School of Nutrition and Translational Research in Metabolism, Maastricht University, Maastricht, The Netherlands.

The project was partially funded by a young investigator research grant provided by the Italian Society of Parenteral and Enteral Nutrition (SINPE, Società Italiana di Nutrizione Parenterale ed Enterale).

Provided by thesis specialist Ridderprint, ridderprint.nl

Printing: Ridderprint

Layout and cover design: Anna Bleeker, persoonlijkproefschrift.nl

Cover illustration image: grmarc, freepik.com

ISBN: 978-94-6416-935-5

Copyright @ Alice Sabatino 2021

All rights reserved. No parts of this thesis may be reproduced, stored in a retrieved system, or transmitted, in any forms or by any means, electronically, mechanically, by photocopying, recording or otherwise, without the prior written permission of the author. For articles published or accepted, the copyright has been transferred to the respective publisher. 


\title{
Quadriceps muscle ultrasound as a new tool for diagnosing muscle wasting in renal diseases
}

\author{
Dissertation \\ To obtain the degree of Doctor at the Maastricht University, on the authority of the Rector \\ Magnificus, Prof.dr. Rianne M. Letschert in accordance with the decision of the Board of Deans, to be \\ defended in public on Monday 13 December 2021 at 13.00 hours.
}

by

Alice Sabatino 


\section{Supervisor}

Prof. dr. J. P. Kooman

\section{Co-Supervisors}

Prof. dr. E. Fiaccadori, Parma University, Parma, Italy

\section{Assessment Committee}

Prof. dr. M.A. Spruit, CIRO, Horn, Netherlands (chair)

Prof. dr. L Cuppari, Fedral University of São Paulo, São Paulo, Brazil

Dr. C.F.M Franssen, University Medical Center Groningen, Groningen, Netherlands Prof. dr. W.H. Mess

Prof. dr. A.M.W.J. Schols 


\section{CONTENT}

Chapter 1 General Introduction

Chapter 2 Reliability of bedside ultrasound for measurement of quadriceps 23 muscle thickness in critically ill patients with acute kidney injury

Chapter 3 Validation by CT scan of quadriceps muscle thickness measurement 37 by ultrasound in acute kidney injury

Chapter 4 Noninvasive evaluation of muscle mass by ultrasonography of quadriceps femoris muscle in End-Stage Renal Disease patients on hemodialysis

Chapter 5 Ultrasound for non-invasive assessment and monitoring of quadriceps muscle thickness in critically ill patients with Acute Kidney Injury

Chapter 6 Quadriceps muscle thickness assessed by ultrasound outperforms conventional anthropometry in the evaluation of mortality risk in hemodialysis patients

Chapter 7 Sarcopenia diagnosed by quadriceps muscle thickness assessed by ultrasound and hand-grip strength predicts mortality in patients on hemodialysis and outperforms bioimpedance spectroscopy.

Chapter 8 General Discussion

Study Summary

Valorization 145

Publications

Acknowledgements

Curriculum vitae 



\section{CHAPTER 1}

General introduction 


\section{DEFINITION AND EPIDEMIOLOGY OF KIDNEY DISEASE (CKD/ESKD AND AKI)}

Major consequences of a reduction in kidney function are a reduction in the kidneys' ability to clean the blood from waste products, an impairment in sodium and water homeostasis leading to hypertension and symptomatic fluid overload, anemia and disturbances in mineral metabolism. A reduction in kidney function can arise either acutely or develop over a more prolonged period of time.

Acute kidney injury (AKI) is characterized by a sudden reduction in kidney function. It is an important complication in hospitalized patients, affecting 10-15\% of all hospital admissions (1). Particularly, critically ill patients in the intensive care unit (ICU) are the most affected subjects, with a prevalence of AKI in this clinical setting exceeding 50\% (2). In the ICU, AKI seldom arises as an isolated complication, but usually is a key component in the multiple organ failure syndrome. It is estimated that about $10 \%$ of critically ill patients with AKI will require kidney replacement therapy (KRT) (3). The presence of AKI independently increases mortality risk by multiple mechanisms such as electrolyte and acid-base disorders, fluid overload, metabolic dysfunction, increased risk of hemorrhagic complications and impaired neutrophil function, reducing the patient's ability to fight infection (1). In addition, even a single episode of AKI is associated with long-term adverse outcomes, such as new onset or worsening of chronic kidney disease (CKD), dialysis dependence, and increased cardiovascular risk (4). According to the Kidney Disease Improving Global Outcome (KDIGO) (5), AKI can be classified in 3 stages based on the severity of loss of kidney function, on the basis of serum creatinine values increase, reduced urine output, or the need to start kidney replacement therapy (KRT) (Table 1). Causes of AKI include, among others, renal hypoperfusion caused by hypovolemic shock or heart failure, nephrotoxin exposure, sepsis, major surgery, intra-abodominal hypertension, rapidly progressive glomerulonephritis, acute interstitial nephritis, and extra- or intra-renal obstruction (6).

On the other hand, CKD is defined as a reduction in the estimated glomerular filtration rate (eGFR) below $60 \mathrm{ml} / \mathrm{min} / 1.73 \mathrm{~m}^{2}$ and/or by the presence of kidney damage for $\geq 3$ months ( 7 ). Kidney damage, next to a decline in GFR, can be confirmed by the presence of albuminuria, defined as albumin/creatinine ratio $>30 \mathrm{mg} / \mathrm{g}$, and/or other markers, such as abnormalities in urinary sediment examination, imaging studies, pathological kidney biopsy (7). CKD severity is classified in 5 stages based on the level of eGFR (Table 1). The global estimated prevalence of CKD ranges from $11.7-15.1 \%$, being patients with end-stage kidney disease (ESKD) needing kidney replacement therapy (KRT) between five and seven million worldwide (8). Because CKD has a direct impact on cardiovascular risk and increases the risk of ESKD, it has been recognized as a leading public health issue, affecting the global morbidity and mortality burden. Principal causes of CKD are diabetes mellitus, hypertension, obesity, and aging. 
Table 1. Acute kidney injury and chronic kidney disease classification

\begin{tabular}{|c|c|c|}
\hline & AKI (KDIGO) & CKD (KDIGO) \\
\hline Stage 1 & $\begin{array}{l}\mathrm{sCr} \geq 1.5 \text { times baseline; or increase of } \geq \\
0.3 \mathrm{mg} / \mathrm{dl} \text { withing } 48 \mathrm{~h} \text {; or urine volume }< \\
0.5 \mathrm{ml} / \mathrm{Kg} \text { for } 6-12 \mathrm{~h}\end{array}$ & $\begin{array}{l}\text { Kidney damage with normal or increased } \\
\text { eGFR }\left(\geq 90 \mathrm{ml} / \mathrm{min} / 1.73 \mathrm{~m}^{2}\right)\end{array}$ \\
\hline Stage 2 & $\begin{array}{l}\mathrm{sCr} \geq 2 \text { times baseline; or urine volume }< \\
0.5 \mathrm{ml} / \mathrm{Kg} \text { for } \geq 12 \mathrm{~h}\end{array}$ & $\begin{array}{l}\text { Kidney damage with mild reduction in } \\
\text { eGFR }\left(60-89 \mathrm{ml} / \mathrm{min} / 1.73 \mathrm{~m}^{2}\right)\end{array}$ \\
\hline Stage 3 & $\begin{array}{l}\mathrm{sCr} \geq 3 \text { times baseline; or increase to } \geq 4.0 \\
\mathrm{mg} / \mathrm{dl} \text {; or or urine volume }<0.3 \mathrm{ml} / \mathrm{Kg} \text { for } \geq \\
24 \mathrm{~h} \text {; or need for } \mathrm{KRT}\end{array}$ & $\begin{array}{l}\text { Moderate reduction in eGFR (Stage } 3 a \\
45-59 \mathrm{ml} / \mathrm{min} / 1.73 \mathrm{~m}^{2} \text {, Stage } 3 \mathrm{~B} 30-44 \mathrm{ml} / \\
\mathrm{min} / 1.73 \mathrm{~m}^{2} \text { ) }\end{array}$ \\
\hline Stage 4 & NA & $\begin{array}{l}\text { Severe reduction in eGFR }(15-29 \mathrm{ml} \\
\left.\mathrm{min} / 1.73 \mathrm{~m}^{2}\right)\end{array}$ \\
\hline Stage 5 & NA & $\begin{array}{l}\text { End-stage kidney disease }(<15 \mathrm{ml} / \\
\mathrm{min} / 1.73 \mathrm{~m}^{2} \text { or dialysis) }\end{array}$ \\
\hline
\end{tabular}

$\mathrm{sCr}$, serum creatinine; KRT, kidney replacement therapy.

\section{EFFECTS OF AKI AND CKD/ESKD ON NUTRITIONAL STATUS OF PATIENTS}

In general, major metabolic and hormonal changes are commonly observed in kidney disease. In the case of AKI, they are determined not only by the syndrome itself, but also by the underlying disease processes, complications and organ dysfunction (9) (Figure 1).

Well-known negative effects of AKI are related to impairments in fluid balance, electrolyte disorders and effects on acid-base metabolism, characterized by metabolic acidosis. However, the syndrome also affects other metabolic pathways, with specific changes in protein, lipid and carbohydrate metabolism. In particular, there is an increase in protein catabolism, which can be caused by multiple factors such as the metabolic acidosis, intra-renal and systemic inflammation associated with AKI and with the critical illness status, the hypercatabolic state caused by the underlying critical illness, the presence of other comorbidities, and by the loss of amino acids and proteins during KRT (9-12). latrogenic factors related to the clinical management of the syndrome can also have negative effects on macronutrient metabolism. Frequently, in critically ill patients with AKI protein intake is erroneously restricted hoping to avoid blood urea nitrogen value increase, while medical nutrition therapy is often insufficient due to delayed start, frequent interruptions, and poor patient's tolerability (9) (Figure 1). All of these frequently coexisting factors may lead to malnutrition, or protein energy wasting (PEW), a term coined in 2008 to describe the particular state of depleted energy and protein pools typical of patients with kidney disease (13). In fact, PEW prevalence in critically ill patients with $\mathrm{AKI}$ can be as high as $42 \%$ (14). 
Figure 1. Protein energy wasting pathophysiology in critically ill patients with AKI

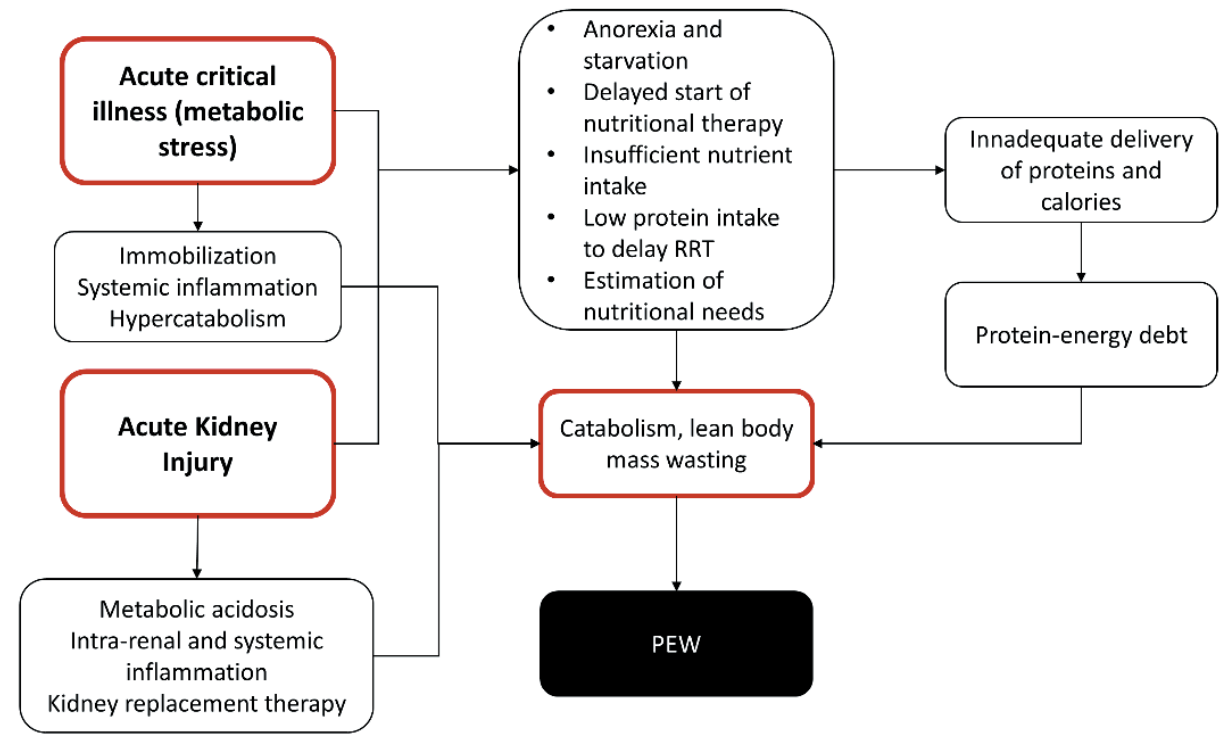

Patients with CKD/ESKD also face muscle loss and worsening of nutritional status, especially in the most advanced stages of the disease, when dialysis as hemodialysis (HD) or peritoneal dialysis (PD) are needed (15-17). In this clinical context, PEW's prevalence can be as high as $75 \%$ in dialysis patients, and $28-48 \%$ in non-dialysis dependent patients, according to CKD stage (18).

The pathophysiology of PEW in CKD/ESKD is multifactorial, and is characterized by the coexistence of increased protein degradation and decreased protein synthesis, leading to negative nitrogen balance (Figure 2). Metabolic acidosis directly triggers systems responsible for intracellular protein degradation (caspase-3 and ubiquitin-proteasome systems (UPS)) (19), and also promotes insulin and growth hormone (GH) resistance (20). In addition, the reduced clearance of cytokines, the dialysis procedure itself and the presence of intestinal dysbiosis and gastrointestinal barrier disruption can lead to a state of chronic low-grade inflammation, which may contribute to an increase in nutritional needs and to anorexia, along with altered blood concentration of appetite mediators and deranged hypothalamus output, leading to an imbalance between the orexigenic/anorexigenic mechanisms that control energy homeostasis $(21,22)$. Other factors include vitamin $D$ deficiency, the loss of amino acids and proteins during $\mathrm{HD}$ and $\mathrm{PD}$, taste abnormalities caused by uremic toxin accumulation, and also socioeconomic conditions, such as poverty and advanced age, also contributing to suboptimal nutrient intake in CKD/ESKD (23). Taken together, those conditions put the patients in a negative protein balance condition resulting in muscle loss, weakness, low physical performance, disability and frailty (24) (Figure 2). 
Figure 2. Mechanisms of protein energy wasting in patients with CKD/ESKD. From (25).

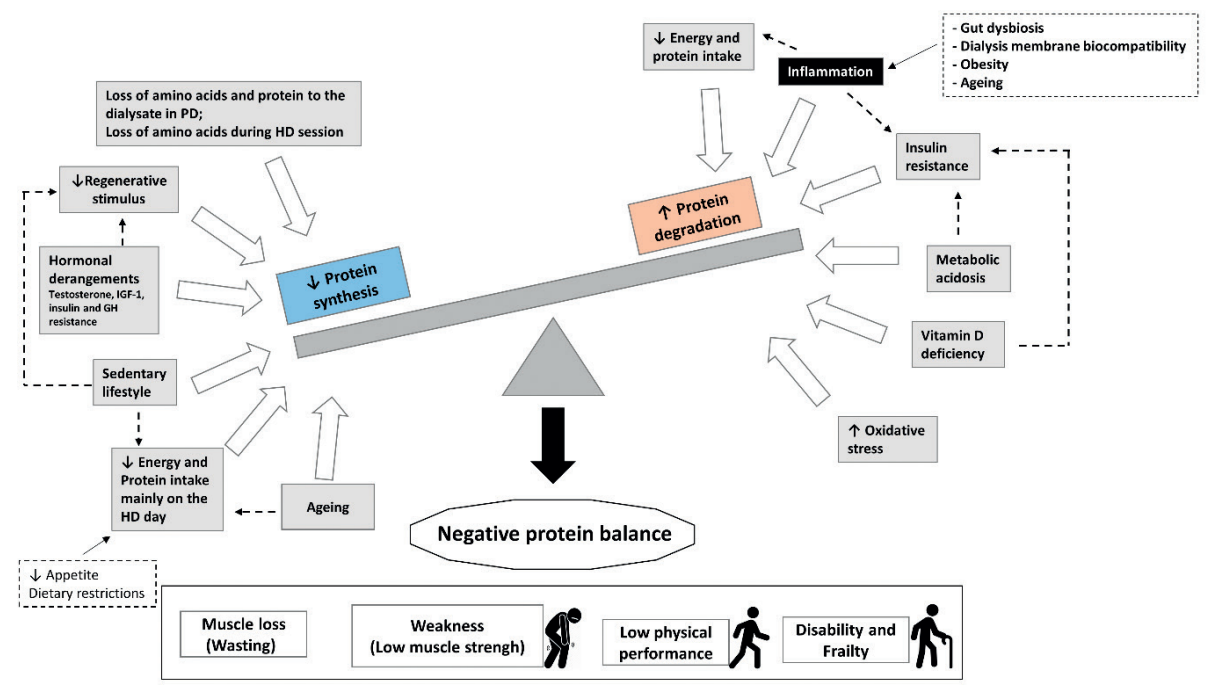

\section{NUTRITIONAL STATUS EVALUATION IN RENAL DISEASE: CURRENT PRACTICE AND RECOMMENDATIONS}

The available recommendations for the assessment of nutritional status in patients with AKI and in patients with CKD/ESKD are toward an integrated approach combining the evaluation of body composition, anthropometric parameters such as height and body weight, blood biochemistry and dietary intake $(13,26,27)$. The International Society of Renal Nutrition and Metabolism (ISRNM) recommends that the diagnosis of PEW be established by the presence of at least one criterion in three out of four categories of nutritional variables (Table 2). Generally, indicators of PEW by the ISRNM diagnostic criteria correlate with increased mortality, decreased appetite and low protein intake $(21,28,29)$.

Table 2. Criteria for the diagnosis of protein energy wasting (13).

a) Reduced levels of biochemical markers of nutritional status such as prealbumin, albumin ( $<3.8 \mathrm{~g} /$ dl) and cholesterol (in patients not using cholesterol reducing medications)

b) Reduced body mass: $\mathrm{BMI}<23 \mathrm{~kg} / \mathrm{m}^{2}$ or unintentional weight loss of $>5 \%$ in 3 months or $10 \%$ in 6 months, reduced fat mass $<10 \%)$;

c) Reduced muscle mass: reduction of muscle mass by $5 \%$ in 3 months or $10 \%$ in 6 months reduced arm muscle circumference (AMC) by $10 \%$ as compared to the $50^{\text {th }}$ percentile of the reference population;

d) Inadequate nutritional intake: spontaneous intake of protein and calories of $<0.8 \mathrm{~g} / \mathrm{Kg} / \mathrm{day}$ for patients with AKI or ESKD in dialysis or $<0.6 \mathrm{~g} / \mathrm{Kg} /$ day in patients with CKD not on dialysis; and spontaneous energy intake $<25 \mathrm{Kcal} / \mathrm{Kg} /$ day respectively for 2 consecutive months

PEW can be diagnosed when at least one criterion in three out of four categories is present 
More recently, new clinical guidelines have been published for the nutritional management of patients with AKI and hospitalized patients with CKD/ESKD (26) and for outpatients with CKD/ ESKD (30). The 2021 ESPEN guideline for hospitalized patients with kidney disease recommends a general nutritional assessment, which includes patient history, report on unintentional weight loss or decrease in physical performance before hospital or ICU admission, dietary intake, physical examination, body composition evaluation to assess skeletal muscle mass, and assessment of muscle strength (26). Differently to the ISRNM criteria (13), the ESPEN guideline does not recommend the use of body mass index (BMI) during hospitalization to assess nutritional status, unless very low $\left(\mathrm{BMI}<18 \mathrm{~kg} / \mathrm{m}^{2}\right)$. This because body weight is easily influenced by fluid imbalance, a frequent finding in patients with kidney disease, which can make BMI unreliable in this clinical setting. Similar concerns arise in the more advanced stages of CKD/ESKD. In fact, the new KDOQI guideline, despite recommending the use of BMI as a mortality predictor, suggests using the classic BMI reference values to assess malnutrition (BMI $<18.5 \mathrm{Kg} / \mathrm{m}^{2}$ ) instead of the more conservative $23 \mathrm{Kg} / \mathrm{m}^{2}$ recommended by the ISRNM criteria. In order to overcome some of BMI limitations, it is further suggested that in patients undergoing dialysis, BMI be evaluated using patient's dry weight, obtained after the dialysis treatment.

Regarding biochemical parameters, despite its sensitivity as a screening and prognostic tool in acute and chronic clinical settings, serum albumin and prealbumin provide limited information regarding nutritional status in the setting of AKI and CKD/ESKD when used in isolation. Serum albumin concentration depends on its synthesis, breakdown, volume distribution, and exchange between intra and extra-cellular spaces, as well as on its losses (31). Both serum albumin and prealbumin are negative acute phase reactants, meaning that during acute illness or chronic inflammation their synthesis is reduced and breakdown is increased, resulting in low serum levels. Still, serum albumin, despite its poor nutritional value in this clinical setting, in combination to the C-reactive protein, an inflammatory marker, can be used for the identification of patients with higher mortality risk and that could benefit from early nutritional intervention (32). Prealbumin, instead, given its shorter half-life (2-3 days) could be cautiously used for the monitoring of the nutritional therapy.

The assessment of body composition, on the other hand, appears to be of major importance both in the case of patients with $\operatorname{AKI}$ as $\operatorname{CKD} / \operatorname{ESKD}(26,30)$. Identifying early muscle loss in both clinical settings is important since it is a well-known predictor of in-hospital mortality and morbidity $(33)$, functional recovery $(34,35)$, disability and hospitalization $(36,37)$ in the ICU-setting; moreover, it is associated with physical disability, worse quality of life, depression, PEW, fracture risk, cardiovascular disease, graft failure and post-operative complications in transplant recipients, as well as increased hospitalization and mortality in patients with CKD/ ESKD (38-45). Important limitations for the successful implementation of body composition assessment in both settings are the lack of reliable bedside tools to assess skeletal muscle mass, and the interference of fluid overload and rapid fluid shifts on currently available conventional methods. The assessment of body composition is also complicated by the fact that various parameters are used to express muscle mass (MM). For instance, fat-free 
mass (FFM), lean body mass (LBM) and MM are not equivalent, although they are often used interchangeably. FFM is the total body mass except the body fat, and it includes LBM and bone mineral tissue. LBM in turn is composed by total water, MM and the fat free part of organs (Figure 3). Since different techniques measure different body compartments, one must identify the body compartment of interest before deciding the method of assessment.

Figure 3. Body composition (conventional model). From (46).

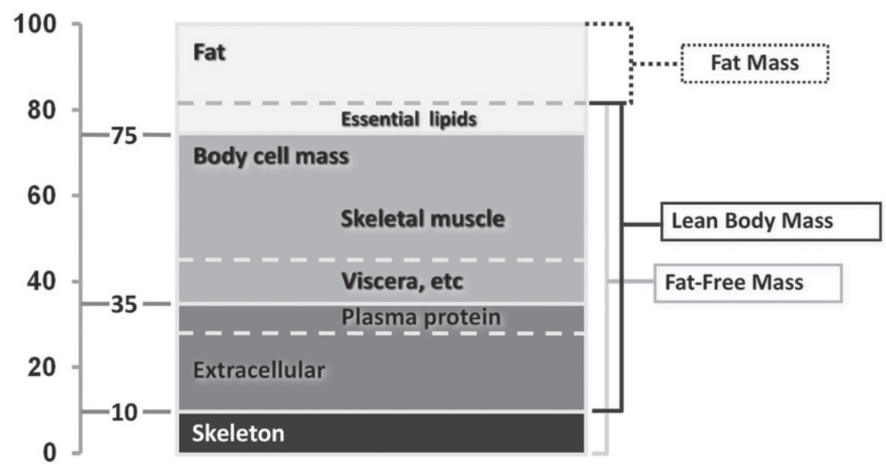

With that in mind, there are many options for the assessment of MM and its proxies. Gold standard methods such as computed tomography (CT) and magnetic resonance imaging (MRI) are impractical to use on a routine basis both in AKI or CKD/ESKD (47). Dual energy X-ray absorptiometry (DEXA), is usually considered a reference method and is also recommended by the new KDOQI guideline for the assessment of body composition, in particular appendicular skeletal MM (ASMM) in patients with CKD/ESKD (30), but may be difficult to perform as often as necessary for the monitoring of ASMM. Moreover, DEXA assumes a constant hydration of the LBM of 0.73 , meaning that results can be influenced by severe fluid overload (48). Nonetheless, when routinely available, DEXA may provide important information regarding changes in body composition and might also serve as calibration for bedside methods.

Currently used and recommended bedside techniques in patients with kidney disease include bioelectrical impedance analysis (BIA) or spectrometry (BIS) and anthropometry (30). However, those methods can be highly influenced by non-nutritional factors. For instance, it has been observed that the use of BIA in patients with AKI reported a 5\% reduction in the FFM when performed after KRT in comparison to measurements performed before (49), showing that BIA can be less reliable in the presence of overhydration. In another study in the same setting, BIA analysis showed the presence of excess percentage of total body water and body fat, suggesting an overestimation of FFM by the fluid overload and a relative increase in FM, which could be instead reflecting a loss of LBM in catabolic patients (50). Considering that critically ill patients will hardly achieve a state of "dry" body weight, the new 2021 ESPEN guideline does not recommend its routine use to assess body composition in hospitalized patients with AKI and fluid imbalance (26). In order to standardize measurements and avoid this kind of problem, 
and considering that in the chronic setting patients are in a more constant condition, the recent KDOQI guidelines on nutrition in CKD recommends to perform BIA/BIS at least 30 minutes after the HD session to allow for redistribution of body fluids (30). Anthropometry is a time-honored method that is also included in the original diagnostic criteria for PEW (51). When performed by a skilled investigator it was able to predict a reduction in MM with an accuracy comparable to instrumental methods (52). However, because of its poor reproducibility and repeatability it is not routinely recommended to be used both in AKI and CKD/ESKD settings (26, 30).

As a potential alternative method, the use of ultrasound (US) for the assessment of MM has been recently proposed. Muscle US is a non-invasive technique easily applicable even in non-collaborative patients, it is economically viable safe and does not require specialized staff nor X-ray exposure (53-56). Quadriceps muscle US, in particular recuts femoris (RF) and vastus intermedius (VI) US, has been successfully studied in other clinical settings. It allows the detection of muscle wasting during ICU stay $(57,58)$, and seems as accurate as CT or MRI in measuring RF diameter in patients with coronary artery disease (CAD) (59), and RF crosssectional area (CSA) (60), and volume (61) in chronic obstructive pulmonary disease (COPD). In particular, mid-thigh thickness (i.e. thickness of the quadriceps femoris) correlates with fat-free mass by DEXA in patients with COPD (62).

\section{STUDY OBJECTIVES}

The present thesis has the objective to study the applicability, validity and prognostic capacity of skeletal muscle US in patients with kidney disease. In particular, the quadriceps rectus femoris (RF) and vastus intermedius (VI) thickness were assessed by US in critically ill patients with $\mathrm{AKI}$ and in patients with ESKD on hemodialysis to:

- Study the inter and intra-rater reliability of the US methodology and if results were affected by acute and rapid fluid shifts due to KRT;

- Study the validity of the quadriceps muscle US in comparison to CT;

- Assess if RF and VI thickness are reduced in patients with ESKD on HD in comparison to healthy controls and hospitalized patients with normal renal function;

- Investigate whether any correlation exists between RF and VI thickness by US and other non-invasive methods for nutritional status evaluation, such as anthropometry and (BIS), handgrip strength (HGS), BMI, malnutrition inflammation score (MIS) and serum albumin;

- Assess if quadriceps muscle US was useful to detect muscle loss in short periods of time;

- Test the prognostic capacity of RF and VI thickness by US in predicting mortality in patients with ESKD on HD. 


\section{THESIS OUTLINE}

Chapter 1 presents a general introduction about the topic.

Chapter 2 reports the study on the reliability of the quadriceps muscle US technique when applied to critically ill patients with AKI

This is the first study in the nephrology setting to use US to assess the quadriceps skeletal muscle. In this study, quadriceps RF and VI thickness of critically ill patients with AKI stage 3 on KRT were measured by two assessors. Intra and interobserver realiability was evaluated using intraclass correlation coefficient (ICC). In addition, the effect of KRT was also studied with measurements performed before and after the KRT.

Chapter 3 reports the results of the study of the validity of quadriceps RF and VI thickness assessed by US in comparison to CT, a gold standard technique for the evaluation of body composition. In this study we validate the proposed US methodology in critically ill patients with AKI. Quadriceps RF and VI thickness of patients were blindly assessed at the same leg sites by both US and CT scans to estimate average difference in thickness, agreement and precision of the US methodology in comparison to CT.

Chapter 4 presents the current status of RF and VI thickness of patients with ESKD on HD in comparison to controls, and it also reports the results of the correlation between US and other parameters to assess nutritional status

This is the first time that quadriceps muscle US was applied in patients with ESKD on HD. By applying quadriceps muscle US and dividing patients based on their BMI, MIS and serum albumin status, we identified that US was sensible enough to identify that RF and VI thickness were reduced in patients with worse nutritional status. In addition, we once again tested whether quadriceps muscle US measurements were affected by the HD procedure. Finally, the correlation of the US methodology with other parameters of nutritional status such as BMI, MIS, mid-arm muscle area (MAMA) and serum albumin was assessed

Chapter 5 details the results of the study of the applicability of quadriceps muscle US in the monitoring of skeletal muscle as a useful tool to detect muscle loss in short periods of time In this short communication study we applied quadriceps RF and VI thickness by US in critically ill patients with AKI at baseline (within $72 \mathrm{~h}$ of hospitalization) and after 5 days to assess whether US was sensible enough to detect changes quadriceps muscle thickness during short periods of time.

Chapter 6. presents the prognostic capacity of quadriceps muscle US in predicting mortality events of patients with ESKD on HD in comparison to anthropometry.

In this study we normalized the US measurements by height ${ }^{2}$ (indexed values) and derived different cut-offs of indexed RF and VI thickness by US based on the distribution (percentiles) of measurements in our sample of patients with ESKD on HD and study the mortality risk 
predictability of 3 different cut-offs (p10, p25 and p50), also in comparison to anthropometry. The hazard-ratio for the risk of mortality was calculated for each studied cut-off and muscle site.

Chapter 7 presents the predictive value for mortality outcome between the indexed thickness of the distal quadriceps VI thickness measured by US with that from the lean tissue index (LTI) measured by BIS, combined with HGS.

In the last study of this thesis we applied the cut-offs of indexed quadriceps muscle US derived from the study described in chapter 6 in combination with HGS to diagnose sarcopenia and compared its prognostic capacity in comparison to sarcopenia as diagnosed by LTI combined with HGS.

Chapter 8 presents a general discussion of the study results. 


\section{REFERENCES}

1. Al-Jaghbeer M, Dealmeida D, Bilderback A, Ambrosino R, Kellum J. Clinical Decision Support for In-Hospital AKI. J Am Soc Nephrol. 2018;29(2):654 - 60.

2. Hoste EA, Bagshaw SM, Bellomo R, Cely CM, Colman R, Cruz DN, et al. Epidemiology of acute kidney injury in critically ill patients: the multinational AKI-EPI study. Intensive Care Med. 2015;41(8):1411-23.

3. Hoste E, Kellum J, Selby N, Zarbock A, Palevsky P, Bagshaw S, et al. Global epidemiology and outcomes of acute kidney injury. Nat Rev Nephrol. 2018;14(10):607-25.

4. Doyle J, Forni L. Acute kidney injury: short-term and long-term effects. Crit Care. 2016;20(1):188.

5. Global KDI, Group. OKAKIW. KDIGO Clinical Practice Guideline for Acute Kidney Injury. . Kidney international. 2012;Suppl 2:1 - 138.

6. Ronco C, Bellomo R, Kellum J. Acute kidney injury. Lancet. 2019;394(10212):1949 - 64.

7. Levey A, Eckardt K, Tsukamoto Y, Levin A, Coresh J, Rossert J, et al. Definition and classification of chronic kidney disease: a position statement from Kidney Disease: Improving Global Outcomes (KDIGO). Kidney Int. 2005;67(6):2089-100.

8. Lv J,Zhang L. Prevalence and Disease Burden of Chronic Kidney Disease. Adv Exp Med Biol. 2019;1165:3-15.

9. Fiaccadori E, Regolisti G, Maggiore U. Specialized nutritional support interventions in critically ill patients on renal replacement therapy. Curr Opin Clin Nutr Metab Care. 2013;16(2):217-24.

10. Rabb H, Griffin MD, McKay DB, Swaminathan S, Pickkers P, Rosner MH, et al. Inflammation in AKI: Current Understanding, Key Questions, and Knowledge Gaps. J Am Soc Nephrol. 2016;27(2):371-9.

11. Bufarah M, de Góes C, Cassani de Oliveira M, Ponce D, Balbi A. Estimating Catabolism: A Possible Tool for Nutritional Monitoring of Patients With Acute Kidney Injury. J Ren Nutr. 2017;27(1):1-7.

12. Patel J, McClain C, Sarav M, Hamilton-Reeves J, Hurt R. Protein Requirements for Critically III Patients With Renal and Liver Failure. Nutr Clin Pract. 2017;32(1_suppl):101S-11S.

13. Fouque D, Kalantar-Zadeh K, Kopple J, Cano N, Chauveau P, Cuppari L, et al. A proposed nomenclature and diagnostic criteria for protein-energy wasting in acute and chronic kidney disease. Kidney Int. 2008;73(4):391-8.

14. Khor BH, Tiong H, Tan S, Abdul Rahman R, Abdul Gafor A. Protein-Energy Wasting Assessment and Clinical Outcomes in Patients with Acute Kidney Injury: A Systematic Review with Meta-Analysis. Nutrients. 2020;12(9):2809.

15. Sabatino A, Regolisti G, Delsante M, Di Motta T, Cantarelli C, Pioli S, et al. Noninvasive evaluation of muscle mass by ultrasonography of quadriceps femoris muscle in End-Stage Renal Disease patients on hemodialysis. Clin Nutr. 2019;38(3):1232-9.

16. Foley RN, Wang C, Ishani A, Collins AJ, Murray AM. Kidney function and sarcopenia in the United States general population: NHANES III. Am J Nephrol. 2007;27(3):279-86. 
17. Giglio J, Kamimura M, Lamarca F, Rodrigues J, Santin F, Avesani C. Association of Sarcopenia With Nutritional Parameters, Quality of Life, Hospitalization, and Mortality Rates of Elderly Patients on Hemodialysis. J Ren Nutr. 2018;28(3).

18. Hanna R, Ghobry L, Wassef O, Rhee C, Kalantar-Zadeh K. A Practical Approach to Nutrition, Protein-Energy Wasting, Sarcopenia, and Cachexia in Patients With Chronic Kidney Disease. Blood purification. 2019.

19. Bailey J, Wang X, England B, Price S, Ding X, Mitch W. The acidosis of chronic renal failure activates muscle proteolysis in rats by augmenting transcription of genes encoding proteins of the ATP-dependent ubiquitin-proteasome pathway. J Clin Invest. 1996;97(6).

20. Hu Z, Wang H, Lee I, Du J, Mitch W. Endogenous glucocorticoids and impaired insulin signaling are both required to stimulate muscle wasting under pathophysiological conditions in mice. J Clin Invest. 2009;119(10).

21. Obi Y, Qader H, Kovesdy CP, Kalantar-Zadeh K. Latest consensus and update on protein-energy wasting in chronic kidney disease. Curr Opin Clin Nutr Metab Care. 2015;18(3):254-62.

22. Wang $\mathrm{XH}$, Mitch WE. Mechanisms of muscle wasting in chronic kidney disease. Nat Rev Nephrol. 2014;10(9):504-16.

23. Sabatino A, Regolisti G, Karupaiah T, Sahathevan S, Sadu Singh BK, Khor BH, et al. Protein-energy wasting and nutritional supplementation in patients with end-stage renal disease on hemodialysis. Clin Nutr. 2017;36(3):663-71.

24. Cohen S, Nathan JA, Goldberg AL. Muscle wasting in disease: molecular mechanisms and promising therapies. Nat Rev Drug Discov. 2015;14(1):58-74.

25. Sabatino A, Cuppari L, Stenvinkel P, Lindholm B, Avesani C. Sarcopenia in chronic kidney disease: what have we learned so far? J Nephrol. 2020.

26. Fiaccadori E, Sabatino A, Barazzoni R, Carrero J, Cupisti A, De Waele E, et al. ESPEN guideline on clinical nutrition in hospitalized patients with acute or chronic kidney disease. Clin Nutr. 2021;40(4):1644-68.

27. Ikizler T, Burrowes J, Byham-Gray L, Campbell K, Carrero J, Chan W, et al. KDOQI Clinical Practice Guideline for Nutrition in CKD: 2020 Update. Am J Kidney Dis. 2020;76(3 Suppl 1):S1-S107.

28. Ikizler TA, Cano NJ, Franch H, Fouque D, Himmelfarb J, Kalantar-Zadeh K, et al. Prevention and treatment of protein energy wasting in chronic kidney disease patients: a consensus statement by the International Society of Renal Nutrition and Metabolism. Kidney Int. 2013;84(6):1096-107.

29. Kalantar-Zadeh K, Cano N, Budde K, Chazot C, Kovesdy C, Mak R, et al. Diets and enteral supplements for improving outcomes in chronic kidney disease. Nat Rev Nephrol. 2011;7(7):369-84.

30. Ikizler T, Burrowes J, Byham-Gray L, Campbell K, Carrero J, Chan W, et al. KDOQI Clinical Practice Guideline for Nutrition in CKD: 2020 Update. Am J Kidney Dis. 2020;76(3 Suppl 1):S1-S10.

31. Jeejeebhoy K. Nutritional Assessment. Gastroenterol Clin North Am. 1998;27(2):347-69. 
32. McCarthy MS, Phipps SC. Special nutrition challenges: current approach to acute kidney injury. Nutr Clin Pract. 2014;29(1):56-62.

33. Puthucheary Z, Montgomery H, Moxham J, Harridge S, Hart N. Structure to function: muscle failure in critically ill patients. The Journal of physiology. 2010;588(23):4641-8.

34. Hiesmayr M. Nutrition risk assessment in the ICU. Curr Opin Clin Nutr Metab Care. 2012;15(2):174-80.

35. Wieske L, Dettling-Ihnenfeldt DS, Verhamme C, Nollet F, van Schaik IN, Schultz MJ, et al. Impact of ICU-acquired weakness on post-ICU physical functioning: a follow-up study. Crit Care. 2015;19:196.

36. Puthucheary ZA, Hart N. Skeletal muscle mass and mortality - but what about functional outcome? Crit Care. 2014;18(1):110.

37. Weijs PJ, Looijaard WG, Dekker IM, Stapel SN, Girbes AR, Oudemans-van Straaten HM, et al. Low skeletal muscle area is a risk factor for mortality in mechanically ventilated critically ill patients. Crit Care. 2014;18(2):R12.

38. Carrero JJ, Chmielewski M, Axelsson J, Snaedal S, Heimburger O, Barany P, et al. Muscle atrophy, inflammation and clinical outcome in incident and prevalent dialysis patients. Clin Nutr. 2008;27(4):557-64.

39. Miyamoto T, Carrero JJ, Qureshi AR, Anderstam B, Heimburger O, Barany P, et al. Circulating follistatin in patients with chronic kidney disease: implications for muscle strength, bone mineral density, inflammation, and survival. Clin J Am Soc Nephrol. 2011;6(5):1001-8.

40. Martinson M, Ikizler TA, Morrell G, Wei G, Almeida N, Marcus RL, et al. Associations of body size and body composition with functional ability and quality of life in hemodialysis patients. Clin J Am Soc Nephrol. 2014;9(6):1082-90.

41. Beddhu S, Pappas LM, Ramkumar N, Samore M. Effects of body size and body composition on survival in hemodialysis patients. J Am Soc Nephrol. 2003;14(9):2366-72.

42. Noori N, Kopple JD, Kovesdy CP, Feroze U, Sim JJ, Murali SB, et al. Mid-arm muscle circumference and quality of life and survival in maintenance hemodialysis patients. Clin J Am Soc Nephrol. 2010;5(12):2258-68.

43. Streja E, Molnar MZ, Kovesdy CP, Bunnapradist S, Jing J, Nissenson AR, et al. Associations of pretransplant weight and muscle mass with mortality in renal transplant recipients. Clin J Am Soc Nephrol. 2011;6(6):1463-73.

44. Oterdoom LH, van Ree RM, de Vries AP, Gansevoort RT, Schouten JP, van Son WJ, et al. Urinary creatinine excretion reflecting muscle mass is a predictor of mortality and graft loss in renal transplant recipients. Transplantation. 2008;86(3):391-8.

45. Giglio J, Kamimura M, Souza N, Bichels A, Cordeiro A, Pinho N, et al. Muscle Mass Assessment by Computed Tomography in Chronic Kidney Disease Patients: Agreement With Surrogate Methods. Eur J Clin Nutr. 2019;73(1).

46. Mundi MS, Patel JJ, Martindale R. Body Composition Technology: Implications for the ICU. Nutr Clin Pract. 2019;34(1):48-58. 
47. Sabatino A, D’Alessandro C, Regolisti G, di Mario F, Guglielmi G, Bazzocchi A, et al. Muscle mass assessment in renal disease: the role of imaging techniques. Quant Imaging Med Surg. 2020;10(8):1672-86.

48. Konings C J, Kooman JP, Schonck M, van Kreel B, Heidendal GA, Cheriex EC, et al. Influence of fluid status on techniques used to assess body composition in peritoneal dialysis patients. Perit Dial Int. 2003;23(2):184-90

49. Ismael S, Savalle M, Trivin C, Gillaizeau F, D’Auzac C, Faisy C. The consequences of sudden fluid shifts on body composition in critically ill patients. Crit Care. 2014;18(2):R49.

50. Ganesan MV, Annigeri RA, Shankar B, Rao BS, Prakash KC, Seshadri R, et al. The protein equivalent of nitrogen appearance in critically ill acute renal failure patients undergoing continuous renal replacement therapy. J Ren Nutr. 2009;19(2):161-6.

51. Fouque D, Kalantar-Zadeh K, Kopple J, Cano N, Chauveau P, Cuppari L, et al. A proposed nomenclature and diagnostic criteria for protein-energy wasting in acute and chronic kidney disease. Kidney Int. 2008;73(4):391-8.

52. Bellafronte NT, Sizoto GR, Vega-Piris L, Chiarello PG, Cuadrado GB. Bed-side measures for diagnosis of low muscle mass, sarcopenia, obesity, and sarcopenic obesity in patients with chronic kidney disease under non-dialysis-dependent, dialysis dependent and kidney transplant therapy. PLoS One. 2020;15(11):e0242671.

53. Connolly B, MacBean V, Crowley C, Lunt A, Moxham J, Rafferty GF, et al. Ultrasound for the assessment of peripheral skeletal muscle architecture in critical illness: a systematic review. Crit Care Med. 2015;43(4):897-905.

54. Sabatino A, Regolisti G, Bozzoli L, Fani F, Antoniotti R, Maggiore U, et al. Reliability of bedside ultrasound for measurement of quadriceps muscle thickness in critically ill patients with acute kidney injury. Clin Nutr. 2017;36(6):1710-5.

55. Regolisti G, Maggiore U, Sabatino A, Gandolfini I, Pioli S, Torino C, et al. Interaction of Healthcare Staff's Attitude With Barriers to Physical Activity in Hemodialysis Patients: A Quantitative Assessment. PloS one. 2018;13(4):e0196313.

56. Battaglia Y, Ullo I, Massarenti S, Esposito P, Prencipe M, Ciancio G, et al. Ultrasonography of Quadriceps Femoris Muscle and Subcutaneous Fat Tissue and Body Composition by BIVA in Chronic Dialysis Patients. Nutrients. 2020;12(5):1388.

57. Reid CL, Campbell IT, Little RA. Muscle wasting and energy balance in critical illness. Clin Nutr. 2004;23(2):273-80.

58. Segers J, Hermans G, Charususin N, Fivez T, Vanhorebeek I, Van den Berghe G, et al. Assessment of quadriceps muscle mass with ultrasound in critically ill patients: intra- and inter-observer agreement and sensitivity. Intensive Care Med. 2015;41(3):562-3.

59. Thomaes T, Thomis M, Onkelinx S, Coudyzer W, Cornelissen V, Vanhees L. Reliability and validity of the ultrasound technique to measure the rectus femoris muscle diameter in older CAD-patients. BMC Med Imaging. 2012;12:7. 
60. Seymour JM, Ward K, Sidhu PS, Puthucheary Z, Steier J, Jolley CJ, et al. Ultrasound measurement of rectus femoris cross-sectional area and the relationship with quadriceps strength in COPD. Thorax. 2009;64(5):418-23.

61. Arbeille P, Kerbeci P, Capri A, Dannaud C, Trappe SW, Trappe TA. Quantification of muscle volume by echography: comparison with MRI data on subjects in long-term bed rest. Ultrasound Med Biol. 2009;35(7):1092-7.

62. Menon MK, Houchen L, Harrison S, Singh SJ, Morgan MD, Steiner MC. Ultrasound assessment of lower limb muscle mass in response to resistance training in COPD. Respir Res. 2012;13:119. 



\section{CHAPTER 2}

Reliability of bedside ultrasound for measurement of quadriceps muscle thickness in critically ill patients with acute kidney injury

Sabatino A, Regolisti G, Bozzoli L, Fani F, Antoniotti R, Maggiore U, Fiaccadori E 


\section{ABSTRACT}

Main problem: In patients with Acute Kidney Injury there is a lack of nutritional variables that can assess nutritional status, more specifically lean body mass (LBM, at the individual level. In this clinical setting, ultrasound (US) of the quadriceps femoris could represent a widely available, non-invasive, affordable, and reliable tool to evaluate skeletal muscle, a clinical surrogate of LBM.

Methods: We performed a cross-sectional observational study in adult critically ill patients with KDIGO stage $3 \mathrm{AKI}$ on dialysis. Quadriceps rectus femoris and vastus intermedius thickness were measured by two assessors. Intra- and interobserver reliability was evaluated using the intraclass coefficient correlation (ICC). The same US measures were obtained before and after dialysis.

Results: We enrolled 34 patients, 22 (65\%) were male and the mean APACHE II score was 22.7 $( \pm 5.6)$. In the intraobserver reliability study, assessor 1 performed 288 paired measurements and assessor 2 performed 430 paired measurements in 34 patients, with an ICC equal to 0.99 and 1.00 , respectively. There were 238 paired measurements (34 patients) in the interobserver reliability study, with an ICC $=0.92$. No difference was found in the measurements obtained before and after dialysis (11.5 (4.2) vs 11.4 (4.1) mm, P=0.2498), independently from acute body weight changes due to fluid removal.

Conclusion: In patients with AKI, US of quadriceps femoris could represent a simple, accurate, and non-invasive method to evaluate quantitative changes in skeletal muscle.

Keywords: Acute kidney injury; Critical illness; Lean body mass; Nutritional status; Renal replacement therapy; Ultrasound. 


\section{INTRODUCTION}

A key problem in AKI, more so in critically ill patients, is the lack of variables allowing nutritional status evaluation/monitoring at the individual level since many factors may significantly interfere with the measurement and interpretation of classical nutritional parameters (1).

Loss of skeletal muscle, the largest store of Lean Body Mass (LBM), is an early effect of critical illness, due to immobilization, catabolism and protein-energy deficit, and is correlated with mortality (2). Different techniques based on radioactive potassium species, Dual X-ray Absorptiometry (DEXA), Computed Tomography (CT) scan and Magnetic Resonance Imaging (MRI) still represent the gold standard for LBM assessment, even though they cannot be routinely used for serial nutritional evaluation in critically ill patients.

Ultrasonography (US) is widely available and easily applicable at the bedside for different skeletal muscle groups, even in critically ill patients (3). It allows the detection of muscle wasting during ICU stay (4), and seems as accurate as CT or MRI in measuring muscle mass (5).

No data are currently available on the reliability of US measurements in patients with AKI. In particular, since total body water is often increased in AKI, peculiar problems may derive from both fluid overload, and the rapid fluid shifts during renal replacement therapy (RRT).

Thus, the primary objective of our study was to investigate the reliability of bedside US in the assessment of quadriceps muscle thickness in critically ill patients with AKI; our secondary objective was to determine whether rapid fluid shifts occurring during RRT might influence US measurements.

\section{MATERIALS AND METHODS}

We conducted a cross-sectional observational study in adult critically ill patients with KDIGO stage $3 \mathrm{AKI}$, consecutively admitted to the renal ICU of the Parma University Hospital. The Institutional Review Board of Parma approved the study (protocol 43943, 03/12/2015). Written informed consent was obtained from patients or their close relatives.

Two assessors (a renal dietician and a nephrologist), who had previously received formal training with at least 100 supervised measurements, performed all the measurements. Quadriceps rectus femoris (QRF) and vastus intermedius (QVI) thicknesses were measured as described by Tillquist et al (6) by B-mode US using a wall tracking system (Philips HD7xe) equipped with a $7.5 \mathrm{MHz}$ linear array transducer. The transducer was placed perpendicular to the long axis of the thigh with abundant use of contact gel and minimal pressure to avoid compression of the muscle. The position and the orientation of the probe were kept steady without using additional equipment in the ICU. The right and left quadriceps values were 
assessed in both legs with the patient lying supine with both knees extended but relaxed and toes pointing to the ceiling. In patients undergoing RRT (conventional 4-hour Hemodialysis (HD) or 6-12 hour sustained low-efficiency dialysis (SLED)) US was performed the start and the end of each session. Both assessors were required to perform two independent measurements at each muscle point (eight measurements per subject), and the mean was used for further analysis. Maximum thickness of each muscle was measured from the femur to the inner edge of the QVI muscle, or from subcutaneous tissue to the inner edge of the QRF muscle (Figure 1) (6-7).

The Wilcoxon signed-rank test was used to analyze the differences in the measurements between assessors, within the same assessor, or before-after RRT. Intra- and interobserver reliability were assessed by the intraclass correlation coefficient (ICC), with values $>0.75$ indicating good to excellent reliability (7), and by the Bland-Altman approach. All values are reported as mean $\pm \mathrm{SD} ; \mathrm{P}<0.05$ was considered significant.

Assuming a minimum ICC value of 0.75 for interobserver reliability, with alpha 0.05 and $80 \%$ power, we estimated that we would need to enroll at least 18 subjects (7). We enrolled 34 subjects to ensure that enough quality images were available.

Figure 1. Assessment of quadriceps femoris thickness using ultrasound. Thickness of each muscle is measured from the femur to the inner edge of the vastus intermedius (VI) muscle, or from subcutaneous tissue to the inner edge of the rectus femoris (RF) muscle.

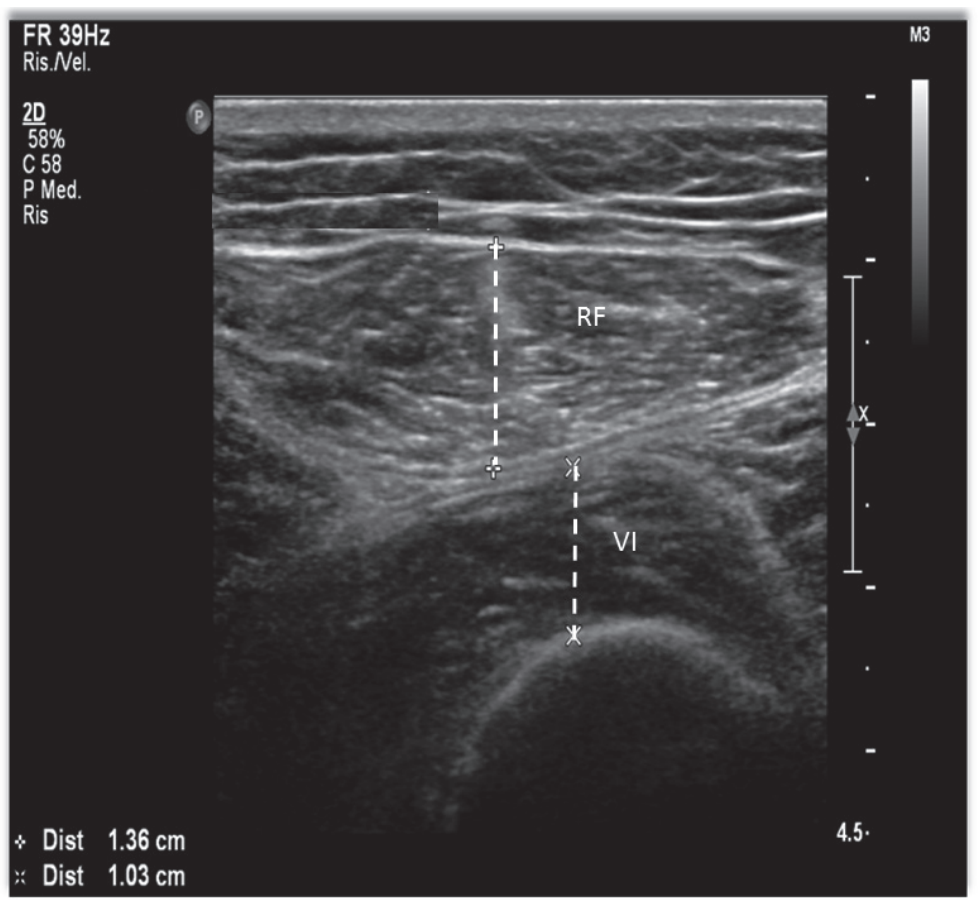




\section{RESULTS}

\section{DEMOGRAPHICS}

Demographic and clinical characteristics of the 34 patients with AKI are reported in Table 1. Most of them were oliguric $(24 / 34,71 \%)$ and on RRT $(29 / 34,85 \%)$ at ICU admission.

- Table 1. Demographics and clinical characteristics of patients with AKI at ICU admission.

\begin{tabular}{ll} 
Parameters & Subjects $(\mathbf{n}=\mathbf{3 4})$ \\
\hline Age (SD) & $75(12)$ \\
\hline Male sex (\%) & $22(65)$ \\
\hline APACHE II (SD) & $22.7(5.6)$ \\
\hline - Oliguria (\%) & $24(71)$ \\
- RRT (\%) & $29(85)$ \\
-Sepsis (\%) & $11(32)$ \\
\hline BMI at ICU admission (SD) & $29.2(6.4)$ \\
\hline Weigth at ICU admission, Kg (SD) & $80.3(14.8)$
\end{tabular}

BMI, body mass index; ER, emergency room, ICU, intensive care unit; RRT, renal replacement therapy; $\mathrm{SD}$, standard deviation.

\section{INTRA- AND INTEROBSERVER RELIABILITY}

Assessor 1 performed 288 pairs of measurements, 72 at each site, with an ICC value of 0.99 (across-site range 0.98 - 0.99) (Table 2A). Assessor 2 performed 430 pairs of measurements (across-site range $106-108$ ), with an ICC value of 1.00 (across-site range $0.97-0.99$ ). No difference was found in the within-assessor measurements: ((assessor 1: 11.5 (3.8) vs 11.5 (3.8) $\mathrm{mm}, 95 \%$ confidence interval $(\mathrm{Cl})$ of the mean difference -0.018 to $0.062, \mathrm{P}=0.42$, assessor 2 : $11.6(4.9)$ vs $11.6(4.9) \mathrm{mm}, 95 \% \mathrm{Cl}$ of the mean difference -0.068 to 0.047 , ( $\mathrm{P}=0.80)$ ) (Table 2B). 
Table 2A. Intraobserver reliability

\begin{tabular}{|c|c|c|c|c|c|}
\hline & & $\mathrm{N}^{\circ}$ measurements & $\begin{array}{l}\text { Between subject } \\
\text { variance }\end{array}$ & $\begin{array}{l}\text { Within subject } \\
\text { variance }\end{array}$ & ICC \\
\hline \multirow{5}{*}{ Assessor 1} & QRFT $1 / 2$ & 72 & 9.229 & 0.1496 & 0.98 \\
\hline & QVIT 1/2 & 72 & 14.20 & 0.1382 & 0.99 \\
\hline & QRFT 2/3 & 72 & 7.91 & 0.1096 & 0.99 \\
\hline & QVIT 2/3 & 72 & 9.26 & 0.0836 & 0.99 \\
\hline & Total & 288 & 14.33 & 0.1225 & 0.99 \\
\hline \multirow{5}{*}{ Assessor 2} & QRFT $1 / 2$ & 108 & 19.32 & 0.5872 & 0.97 \\
\hline & QVIT 1/2 & 108 & 30.94 & 0.3535 & 0.99 \\
\hline & QRFT 2/3 & 108 & 10.11 & 0.3106 & 0.97 \\
\hline & QVIT 2/3 & 106 & 22.67 & 0.2073 & 0.99 \\
\hline & Total & 430 & 23.70 & 0.3654 & 1.00 \\
\hline
\end{tabular}

ICC, intraclass correlation coefficient; QRFT, quadriceps rectus femoris thickness; QVIT, quadriceps vastus intermedius thickness.

Table 2B. Differences within assessors' measurements.

\begin{tabular}{llllll} 
& & $\mathbf{N}^{\circ}$ measurements & Measurement 1 & Measurement 2 & P \\
\hline \multirow{4}{*}{ Assessor 1 } & QRFT 1/2 & 72 & $14.83(3.04)$ & $14.81(1.14)$ & 0.5155 \\
\cline { 2 - 6 } & QVIT 1/2 & 72 & $11.12(3.77)$ & $11.12(3.69)$ & 0.4039 \\
\cline { 2 - 6 } & QRFT 2/3 & 72 & $11.03(2.81)$ & $11.00(2.76)$ & 0.3291 \\
\cline { 2 - 6 } & QVIT 2/3 & 72 & $9.13(3.04)$ & $9.09(2.96)$ & 0.2949 \\
\cline { 2 - 6 } & Total & 288 & $11.53(3.79)$ & $11.51(3.76)$ & 0.4208 \\
\hline \multirow{3}{*}{ Assessor 2 } & QRFT 1/2 & 108 & $14.16(4.40)$ & $14.25(4.32)$ & 0.2378 \\
\cline { 2 - 6 } & QVIT 1/2 & 108 & $12.12(5.56)$ & $12.13(5.63)$ & 0.6869 \\
\cline { 2 - 6 } & QRFT 2/3 & 108 & $10.16(3.18)$ & $10.15(3.03)$ & 0.7645 \\
\cline { 2 - 6 } & QVIT 2/3 & 106 & $9.76(4.76)$ & $9.71(4.71)$ & 0.4100 \\
\cline { 2 - 6 } & Total & 430 & $11.56(4.87)$ & $11.57(4.85)$ & 0.7983
\end{tabular}

QRFT, quadriceps rectus femoris thickness; QVIT, quadriceps vastus intermedius thickness. Mean (SD), values in $\mathrm{mm}$.

The two assessors performed 238 paired measurements (across-site range 56 - 62) with an ICC value of 0.92 (across-site range $0.88-0.93$ ) (Table 3A). Although between-investigator differences in measurements were statistically significant at all sites, the mean absolute value of the difference was approximately $0.4 \mathrm{~mm}(10.7$ (3.4) vs 10.3 (3.3) mm, 95\% Cl of the mean difference $0.28-0.54, \mathrm{P}<0.0001$ ) (Table $3 \mathrm{~B}$ ), and was therefore clinically insignificant. 
Table 3A. Interobserver reliability

\begin{tabular}{lllll} 
& $\mathbf{N}^{*}$ measurements & Between subject variance & Within subject variance & ICC \\
\hline QRFT 1/2 & 56 & 9.4 & 1.3 & 0.88 \\
\hline QVIT 1/2 & 62 & 12.7 & 1.5 & 0.89 \\
\hline QRFT 2/3 & 56 & 6.0 & 0.8 & 0.88 \\
\hline QVIT 2/3 & 62 & 8.8 & 0.6 & 0.93 \\
\hline Total & 238 & 11.7 & 1.1 & 0.92
\end{tabular}

ICC, intraclass correlation coefficient; QRFT, quadriceps rectus femoris thickness; QVIT, quadriceps vastus intermedius thickness.

Table 3B. Differences between assessor's measurements

\begin{tabular}{lllll} 
& $\mathbf{N}^{\mathbf{0}}$ measurements & Assessor 1 & Assessor 2 & P \\
\hline QRFT 1/2 & 56 & $13.3(3.1)$ & $13.1(3.01)$ & 0.0361 \\
\hline QVIT 1/2 & 64 & $10.7(3.6)$ & $10.3(3.4)$ & 0.0150 \\
\hline QRFT 2/3 & 56 & $9.6(2.5)$ & $9.3(2.2)$ & 0.005 \\
\hline QVIT 2/3 & 62 & $9.2(2.9)$ & $8.7(2.8)$ & $<0.0001$ \\
\hline Total & 238 & $10.7(3.4)$ & $10.3(3.3)$ & $<0.0001$
\end{tabular}

QRFT, quadriceps rectus femoris thickness; QVIT, quadriceps vastus intermedius thickness. Mean (SD), values in $\mathrm{mm}$.

\section{BEFORE-AFTER RRT MEASUREMENTS}

Measurements before and after dialysis are reported in table 4. Assessor 1 performed 294 measurements in 39 sessions, while assessor 2 performed 306 measurements in 40 sessions. Sustained low-efficiency dialysis accounted for $38 / 70$ sessions ( $54 \%$ ), with a mean duration of 408 (SD 102) minutes (range 240 - 720 minutes); intermittent HD accounted for the remaining 32 treatments, with a mean duration of 218 (SD 42) minutes (range 120 - 240 minutes).

No difference was found in the measurements obtained before and after RRT, either for the average value or for each assessor (average: 11.5 (4.2) vs $11.4(4.1) \mathrm{mm}, \mathrm{P}=0.25$; assessor 1 : 11.9 (4.0) vs 11.7 (3.8) mm, P = 0.25; assessor 2: 11.1 (4.5) vs 11.0 (4.4) $\mathrm{P}=0.63$ ) (Table 4), even when only RRT sessions with negative weight change were considered $n=62$, (median weight change per session $-1.5 \mathrm{Kg}$, range -0.5 to $-3.0 \mathrm{Kg}$ ) (Table 5). 
Table 4. Measurements before and after RRT

\begin{tabular}{|c|c|c|c|c|c|}
\hline & & $\mathrm{N}^{\circ}$ Measurements & Before & After & $\mathbf{P}$ \\
\hline \multirow{5}{*}{ Assessor 1} & QRFT $1 / 2$ & 72 & $15.2(3.5)$ & $14.9(3.6)$ & 0.1165 \\
\hline & QVIT 1/2 & 74 & $12.0(4.2)$ & $11.9(4.0)$ & 0.1827 \\
\hline & QRFT 2/3 & 72 & $11.5(2.9)$ & $11.3(2.7)$ & 0.0660 \\
\hline & QVIT 2/3 & 74 & $9.0(2.3)$ & $9.0(2.1)$ & 0.2390 \\
\hline & Total & 294 & $11.9(4.0)$ & $11.7(3.8)$ & 0.2530 \\
\hline \multirow{5}{*}{ Assessor 2} & QRFT $1 / 2$ & 75 & $13.6(4.2)$ & $13.5(4.0)$ & 0.4985 \\
\hline & QVIT 1/2 & 79 & $11.5(5.1)$ & $11.5(5.0)$ & 0.3200 \\
\hline & QRFT 2/3 & 75 & $9.8(2.9)$ & $9.8(2.9)$ & 0.1264 \\
\hline & QVIT 2/3 & 77 & $9.5(4.3)$ & $9.4(4.1)$ & 0.2348 \\
\hline & Total & 306 & $11.1(4.5)$ & $11.0(4.4)$ & 0.6309 \\
\hline \multirow{5}{*}{ Total } & QRFT $1 / 2$ & 147 & $14.4(3.9)$ & $14.2(3.9)$ & 0.3015 \\
\hline & QVIT 1/2 & 153 & $11.7(4.7)$ & $11.7(4.5)$ & 0.7918 \\
\hline & QRFT 2/3 & 147 & $10.6(3.0)$ & $10.5(2.9)$ & 0.1733 \\
\hline & QVIT 2/3 & 153 & $9.2(3.4)$ & $9.2(3.3)$ & 0.7564 \\
\hline & Total & 600 & $11.5(4.2)$ & $11.4(4.1)$ & 0.2498 \\
\hline
\end{tabular}

QRFT, quadriceps rectus femoris thickness; QVIT, quadriceps vastus intermedius thickness; RRT, renal replacement therapy. Mean (SD), data in $\mathrm{mm}$.

Table 5. Measurements before and after RRT only in patients with negative weight change.

\begin{tabular}{ccccc} 
& $\mathbf{N}^{\circ}$ Measurements & Before & After & P \\
\hline Assessor 1 & 231 & $11.7(4.0)$ & $11.6(3.9)$ & 0.0967 \\
\hline Assessor 2 & 258 & $11.5(4.6)$ & $11.4(4.5)$ & 0.2544
\end{tabular}

Mean (SD), data in $\mathrm{mm}$.

The absolute interobserver and before-after variability, as expressed by the limits of agreement ( $\pm 1.96 \mathrm{SD}$ ), was $4.1 \mathrm{~mm}$ (range 3.1 to $4.8 \mathrm{~mm}$ for each site), and $3.0 \mathrm{~mm}$ (range 2.3 to $3.8 \mathrm{~mm}$ ), respectively (Table 6). The distributions of the differences are illustrated in Figures 2 and 3. 
Table 6. Interobserver and before- after RRT variability as expressed by the limits of agreement.

Parameter

Interobserver variability $(\mathrm{mm})$
Bland-Altman: $2{ }^{\star} 1.96 *$ SD

\begin{tabular}{ll} 
QRFT 1/2 & 3.0 \\
\hline QVIT 1/2 & 2.9 \\
\hline QRFT 2/3 & 3.8 \\
\hline QVIT 2/3 & 2.3 \\
\hline Total & 2.8 \\
\hline QRFT 1/2 & 4.1 \\
\hline QVIT 1/2 & 4.5 \\
\hline QRFT 2/3 & 4.8 \\
\hline QVIT 2/3 & 3.6 \\
\hline Total & 3.1
\end{tabular}

QRFT, quadriceps rectus femoris thickness; QVIT, quadriceps vastus intermedius thickness; RRT, renal replacement therapy; SD, standard deviation.

Figure 2. Bland-Altman plots for agreement between assessors. The x-axis shows the mean of two measurements performed by each assessor. The $y$-axis shows the difference between means of these values for each assessor. The horizontal lines parallel to the $x$-axis represent the mean difference and limits of agreement for that muscle parameter.

QRFT 1/2

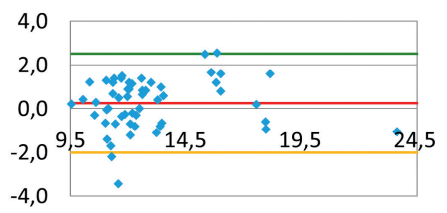

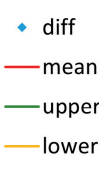

QRFT 2/3
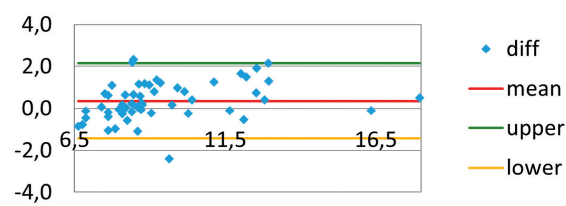

QVIT 1/2

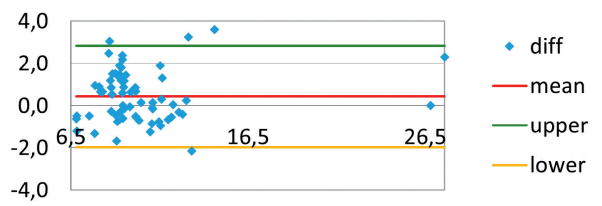

QVIT 2/3

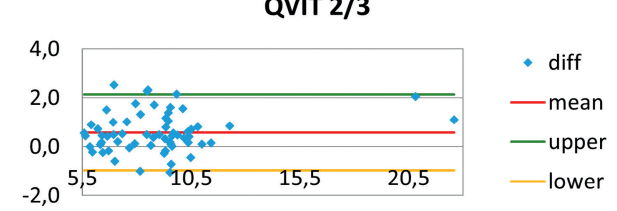

All sites

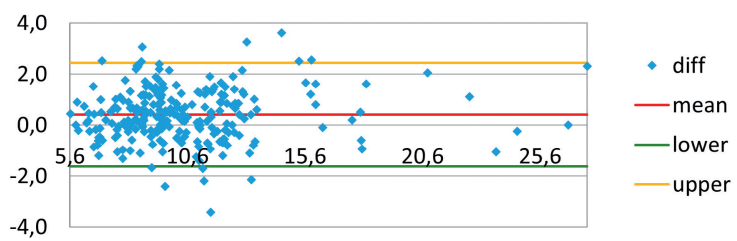


Figure 3. Bland-Altman plots for agreement between measurements performed before and after RRT. The $x$-axis shows the mean of two values. The $y$-axis shows the difference between means of these values. The horizontal lines parallel to the $x$-axis represent the mean difference and limits of agreement for that muscle parameter.

QRFT 1/2

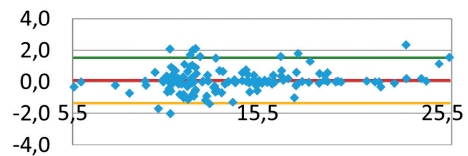

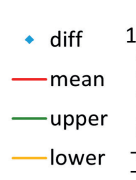

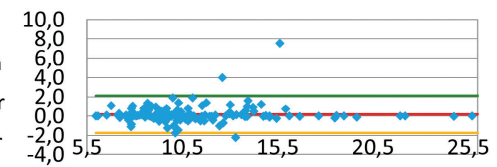

- diff

-mean

- upper

- lower
QRFT 2/3

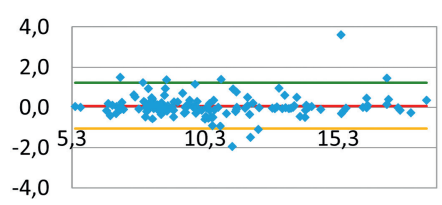

QVIT 2/3

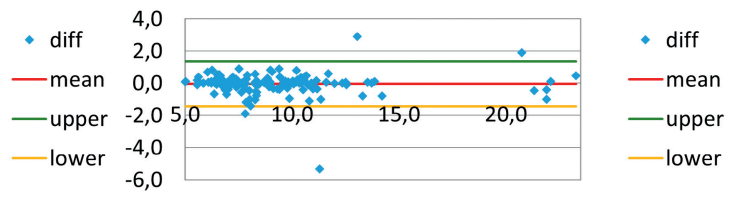

\section{All sites}

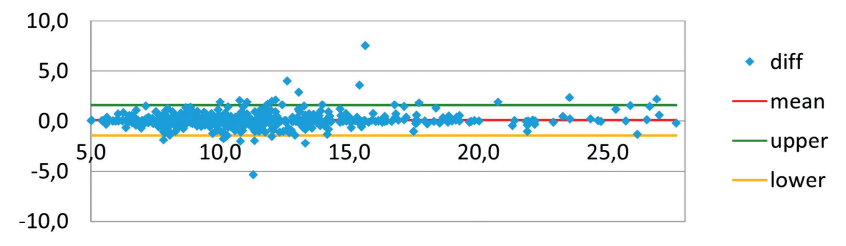




\section{DISCUSSION}

Our data confirm that US assessment of peripheral skeletal muscle has excellent reliability in critically ill patients with KDIGO stage $3 \mathrm{AKI}$, as previously shown in normal subjects (6).

The ICC value obtained in the interobserver reliability analysis was high (0.92), similar to the data of a very recent study on $149 \mathrm{ICU}$ patients $(I C C=0.94)(8)$. The mean difference in muscle thickness values between assessors was statistically significant because of the large number of measurements performed by each assessor. However, this difference was approximately $0.4 \mathrm{~mm}$, which is not clinically relevant, suggesting that the technique is reliable even when different operators are involved. Furthermore, no significant difference was detected between pairs of measurements performed by each operator.

There is a strong need for reliable tools to evaluate and monitor nutritional status, particularly LBM, in patients with AKI. In this regard, nutritional parameters evaluated should be clinically relevant, repeatedly measurable at different time points at the bedside without patient discomfort, not influenced by non-nutritional factors, noninvasive and inexpensive; on these grounds, ultrasound techniques are clearly appealing.

Ultrasound techniques are increasingly used to assess muscle mass and quality, and have been applied in the ICU setting $(4,8,9)$. To our knowledge, this is the first study assessing the reliability of this approach in critically ill patients with AKI on RRT. In our study, we could also demonstrate that US measurements of the quadriceps femoris muscle are not influenced by rapid fluid shifts, as it had been suggested by a previous study in a small number of critically ill patients with multiple organ failure (10).

Our study has several limitations. Firstly, we could not evaluate muscle cross-sectional area (CSA) or echogenicity. In fact, our probe length did not allow the evaluation of rectus femoris area, nor was a dedicated software to evaluate muscle echogenicity available. Secondly, we did not compare our measurements with those obtained with a gold standard method. A very good correlation with quadriceps muscle assessed by MRI has been previously demonstrated for US on subjects with long-term bed rest (5). While the recent VALIDIUM study has demonstrated only a moderate correlation between the quadriceps muscle layer thickness and CSA of the skeletal muscles measured by CT scan at the level of the third lumbar vertebra in critically ill patients (8). Thus, our study may prompt further validation studies directly comparing US with gold standard methods assessing muscle mass in the same district.

In conclusion, our data suggest that US could represent an easy, accurate, and noninvasive method to evaluate quantitative changes of skeletal muscle in patients with AKI. Ultrasonography can be easily used at the bedside and, with appropriate training, it can also be performed by non-specialists. 


\section{DISCLOSURE}

Alice Sabatino is the recipient of a young investigator research grant by the Italian Society of Parenteral and Enteral Nutrition (SINPE, Società Italiana di Nutrizione Parenterale ed Enterale) for the project: "Valutazione nutrizionale nell'insufficienza renale mediante ecografia del muscolo quadricipite femorale" (Nutritional assessment of patients with chronic kidney disease and acute kidney injury through ultrasound of the quadriceps femoris muscle)". 


\section{REFERENCES OF CHAPTER 2}

1. Fiaccadori E, Regolisti G, Maggiore U. Specialized nutritional support interventions in critically ill patients on renal replacement therapy. Curr Opin Clin Nutr Metab Care. 2013; 16: 217-224.

2. Puthucheary ZA, Rawal J, McPhail M et al. Acute skeletal muscle wasting in critical illness. JAMA. 2013; 310: 1591-1600.

3. Connolly B, MacBean V, Crowley C et al. Ultrasound for the assessment of peripheral skeletal muscle architecture in critical illness: a systematic review. Crit Care Med. 2015; 43: 897-905

4. Segers J, Hermans G, Charususin $\mathrm{N}$ et al. Assessment of quadriceps muscle mass with ultrasound in critically ill patients: intra- and inter-observer agreement and sensitivity. Intensive Care Med. 2015; 41: 562-563.

5. Arbeille P, Kerbeci P, Capri A, Dannaud C, Trappe SW, Trappe TA. Quantification of muscle volume by echography: comparison with MRI data on subjects in long-term bed rest. Ultrasound Med Biol. 2009; 35: 1092-1097

6. Tillquist M, Kutsogiannis DJ, Wischmeyer PE et al. Bedside ultrasound is a practical and reliable measurement tool for assessing quadriceps muscle layer thickness. JPEN J Parenter Enteral Nutr. 2014; 38: 886-890.

7. Sarwal A, Parry SM, Berry MJ et al. Interobserver Reliability of Quantitative Muscle Sonographic Analysis in the Critically III Population. J Ultrasound Med. 2015; 34: 1191-1200

8. Paris MT, Mourtzakis M, Day A et al. Validation of bedside ultrasound of muscle layer thickness of the quadriceps in the critically ill patient (VALIDIUM study): a prospective multicenter study. JPEN J Parenter Enteral Nutr. 2016; in press DOI: 10.1177/0148607116637852

9. Parry SM, El-Ansary D, Cartwright MS et al. Ultrasonography in the intensive care setting can be used to detect changes in the quality and quantity of muscle and is related to muscle strength and function. J Crit Care. 2015; 30: 1151.e9-1151.e14

10. Campbell IT, Watt T, Withers D et al. Muscle thickness, measured with ultrasound, may be an indicator of lean tissue wasting in multiple organ failure in the presence of edema. Am J Clin Nutr. 1995; 62: 533-539 



\section{CHAPTER 3}

\section{Validation by CT scan of quadriceps muscle thickness measurement by ultrasound in acute kidney injury}

Sabatino A, Regolisti G, Di Mario F, Ciuni A, Palumbo A, Peyronel F, Maggiore U, Fiaccadori E 


\section{ABSTRACT}

Background: Accelerated muscle wasting still represents a major issue in critically ill patients. However, a key problem in the intensive care unit is the lack of adequate tools for bedside evaluation of muscle mass. Moreover, when Acute Kidney Injury (AKI) coexists, fluid overload and/or rapid fluid shifts due to renal replacement therapies that frequently occur and may interfere with muscle mass assessment. The purpose of this study is to validate muscle ultrasound (US) by a gold standard (muscle CT scan) for the assessment of quadriceps muscle thickness in critically ill patients with AKI.

Methods: Quadriceps rectus femoris thickness and quadriceps vastus intermedius thickness of critically ill patients with AKI were blindly assessed at the same leg sites by both US and computed tomography (CT) scan. Using bivariate mixed-model linear regression analysis, we estimated, average difference in thickness between measurement sites, agreement (differential and proportional bias) of US compared to CT, and precision of the two methods, and eventually performed Bland-Altman analysis for repeated measurements on pooled results.

Results: We analyzed 233 couples of measurements (30 patients). Average muscle thickness ranged between 1.0 and 1.6, depending on the measurement site. When comparing US to CT, both the observed differential bias (between +0.04 and $+0.26 \mathrm{~cm}$ depending on the muscle site) and the proportional bias (between $82 \%$ and $98 \%$ of the reference values, depending on the muscle site) were not statistically significant. However, precision analysis showed that US scan tended to be slightly less precise in comparison to CT. Bland-Altman analysis on pooled results showed that the $95 \%$ limits of agreement between the US and CT were narrow, ranging from -0.34 to $+0.36 \mathrm{~cm}$.

Conclusion: In critically ill patients with AKI, quadriceps muscle thickness assessment based on US is unbiased, although it occurs with a minor loss of precision compared to CT.

Key words: acute kidney injury, computerized tomography scan, critical illness, intensive care unit, muscle mass, ultrasound 


\section{INTRODUCTION}

Critically ill patients almost inevitably suffer an important and accelerated skeletal muscle loss already occurring in the first few days of intensive care unit (ICU) stay (1-3).

This pathological condition, could represent a major cause of delayed weaning from mechanical ventilation, and is a well-known predictor of increased in-hospital mortality and morbidity $(4,5)$. Muscle mass at the time of ICU admission and discharge also has a significant impact both on the patients' outcomes and on the degree of functional recovery achieved in the medium- and long term in survivors $(6,7)$. Finally, low muscle mass is associated with increased disability and higher risk for discharge into long-term care facilities $(8,9)$.

The pathogenesis of muscle wasting in the ICU is complex. Many factors are involved, such as undernutrition, increased catabolism due to stress-related cortisol response and, systemic inflammation, acute comorbidities (trauma, burns etc.), immobilization and the use of sedation/neuromuscular blockers (2).

A key problem in the critically ill is the lack of adequate tools for routine muscle mass evaluation and monitoring at the bedside (10). In fact, the deranged metabolic milieu, as well as fluid overload and the acute phase response may significantly interfere with the use of conventional methods for muscle mass evaluation, such as anthropometry and bioimpedance analysis (10). This is even more true in case of Acute Kidney Injury (AKI) (10), a frequent complication in this clinical setting, especially when sepsis coexists or develops $(11,12)$. Even though Dual Energy X-ray Absorptiometry (DEXA), Computed Tomography scan (CT) and Magnetic Resonance Imaging (MRI) are considered the reference standard techniques for the assessment of skeletal muscle mass and body composition, they cannot be used routinely with this aim in the ICU (13).

The use of ultrasound (US) for the assessment of muscle mass has aroused considerable interest in recent years. Muscle US is a noninvasive technique easily applicable at the bedside even in non-collaborative patients, it is economically advantageous, viable, safe and does not require specialized staff or X-ray exposure (14-16). Its reliability has been recently well documented in critically ill patients with AKI (15). In addition, the US technique seems to be poorly influenced by the rapid and relevant fluid shifts typical of patients with AKI on Renal Replacement Therapies (RRT). In fact, no differences were found in these patients between measurements performed before and after RRT sessions (15), and this features have also been confirmed in end-stage renal patients on conventional hemodialysis (16). However, to the best of our knowledge, a formal validation study of US assessment of skeletal muscle against a gold standard technique in the setting of AKI has never been performed. Pending the results of such study, US measurement of muscle mass may not be safely used for clinical practice in AKI, and US measurement numeric values may not be compared across different studies. 
With this background, we aimed at validating US for the assessment of quadriceps femoris thickness in critically ill patients with AKI, using CT scan as the reference method. To this purpose, we applied a novel analytic approach that allows a detailed assessment, at each muscle site, of the amount of differential and proportional bias between US and CT measurements, as well as the precision of US measurements in comparison to CT.

\section{METHODS}

We conducted a cross-sectional observational study in the Renal ICU of the Parma University Hospital. Procedures were performed in accordance to the Helsinki declaration. Informed consent was obtained from patients or their next of kin. The study was approved by the Local Ethics Committee Area Vasta Emilia Nord (AVEN). Adult patients with a diagnosis of AKI on the basis of the KDIGO criteria (17) consecutively admitted from March 15, 2017 to March 15, 2018, and in whom CT scan was performed for any medical reason, were eligible. We used the STARD checklist when writing our report (18).

\section{- $\quad$ US technique}

Quadriceps rectus femoris thickness (QRFT) and quadriceps vastus intermedius thickness (QVIT) were measured by B-mode ultrasonography, wall tracking ultrasound system (Philips hd7xe) with a 7.5 MHz linear array transducer (L12-3 transducer), as previously described in detail (15). The right and left quadriceps values were assessed in both legs with the patient lying in a supine position with both knees extended but relaxed and toes pointing to the ceiling. A metric tape was used to identify and mark the two reference points in each leg. QRFT and QVIT were measured at the border between the upper third (RF,Prox; VI,Prox) and lower two-thirds (RF,Dist; VI,Dist) between the anterior superior iliac spine (ASIS) and the upper pole of the patella $(15,19)$. The transducer was placed perpendicular to the long axis of the thigh with a large amount of gel and with no pressure to avoid compression of the muscle. The assessor was positioned on the side of the patient while performing the measurements, and was allowed to tilt the probe to obtain the best possible image, in which RF and VI would be aligned and centered. Measurements were performed directly on the ultrasound machine while obtaining the images. The vertical diameter of the muscles was measured at the widest point, on the inner edge of the muscle fascia. All thickness measurements are expressed in centimeters. Ultrasound measurements were performed immediately before or not later than $12 \mathrm{~h}$ after CT scan (the median time lapse between US and CT scans measurements were $3 \mathrm{~h}$ after the $\mathrm{CT}$ ). The US assessor was blinded to the $\mathrm{CT}$ scan results.

\section{- $\quad$ CT scan technique}

CT scans were performed using a Somaton Definition Flash CT scanner. Patients that needed CT scan for any medical reason were eligible. When the physician decided a patient needed a CT scan, the responsible researcher contacted the reference radiologist for the study protocol, which was responsible for arranging the radiological measurements. At the time 
of CT scan, the exam was extended to obtain a single slice for each point of reference in the legs (two images per patient). There was no limitation regarding the type of CT needed by the patient (abdominal, lung, lung+abdominal or lower limbs). Scans were taken exactly at the same sites used for the US. Sites utilized for CT scans and US measurements were marked with a temporary plastic electrode. Rectus femoris and vastus intermedius thicknesses were calculated using the Siemens Magic View VE 40 software, after manual outline with a movable cursor. The radiologist performing QRFT and QVIT assessments was blinded to US measurements.

Demographics, clinical data, renal function and outcome: data were collected as per institutional routine at the time of ICU admission and during ICU stay, with special regard to demographic, clinical and laboratory data, renal function, acute and chronic comorbidities, severity of illness (APACHE II and SOFA scores), and data on renal replacement therapy.

\section{STATISTICAL ANALYSIS}

Validation studies require at least 100 measurements (20). In our study, we planned the enrollment of 30 patients with 8 measurements per patient (four in each leg). Stata SE release 15 (2017, StataCorp, College Station, Tx, USA) was used for all the analyses which we carried out in three steps. First, we fitted a bivariate mixed model to joint CT and US data using the Stata program gsem with patients included as random effects, in order to estimate the differences in muscle thickness between muscle types (VI vs RF), different positions (distal vs proximal) and different sides (left vs right). Since we did not find any difference between left and right side, in the subsequent analyses we regarded the two sides as duplicate measurements of a constant value. The lack of difference between the right and left side was an expected finding since none of the patients had history of surgery on lower limbs and none of them were athletes. In a second stage, we used the approach of Taffé (21) to consistently quantify, for each muscle type and position, the amount of differential and proportional biases between US and CT (which we displayed as "bias plots") and to compare precision between the two methods (which was displayed as "precision plots"). These analyses, which were carried out with the program biasplot (22), allowed for heteroscedastic measurement errors (i.e. measurement error changing with the level of the true -latent- value of muscle thickness). Since the differential and proportional bias between US and CT were non-statistically significant in any muscle type or positions, in the final stage we pooled all the data together and drew a Bland-Altman plot with $95 \%$ limits of agreement. We calculated those limits assuming that the observed differences between US and CT resulted from the sum of the overall mean difference (bias), of random-subjects effect (heterogeneity) and of random error within the subject (23). For the purpose, we calculated the paired difference between US and CT and fitted a mixed model with muscle type and position as fixed effects. 


\section{RESULTS}

Thirty-four patients were eligible for the study. We enrolled 30 critically ill patients ( 17 males) with AKI, and we obtained 233 coupled measurements ( 1 patient had all his 4 proximal measurements excluded because the $\mathrm{CT}$ image was obtained on the wrong place, 1 patient was morbidly obese and his proximal VI muscle in both legs were not visible, and in 1 patient the image obtained of his proximal VI muscle on the right leg had an artifact that did not allow for a measurement). Four patients were excluded because CT scan images were not available due to technical or clinical problems. Patients were studied within 5 days (range 1 - 19) of the diagnosis pf AKI. The mean age of the cohort was $70( \pm 13.6)$ years. Clinical and demographic data are shown in Table 1. The average APACHE II score was $21( \pm 6)$; the median SOFA score was $7(2-16)$. The majority of patients (17/30,57\%) had chronic kidney disease (CKD) prior to the ICU admission (AKI on CKD). Sixty percent of patients (18/30) underwent renal replacement therapy (RRT) within the first $24 \mathrm{~h}$ after ICU admission. Sixty-seven percent of all patients were oliguric and $27 \%$ were septic. Two hundred thirty-three couples of measurements were analyzed.

Table 1. Demographic and clinical variables

\begin{tabular}{|c|c|}
\hline Variables & Values \\
\hline Age & $70( \pm 13.6)$ \\
\hline Male gender (\%) & $17 / 30(57)$ \\
\hline Body weight at admission (Kg) & $82( \pm 25)$ \\
\hline $\mathrm{BMI}\left(\mathrm{Kg} / \mathrm{m}^{2}\right)^{\star}$ & $29( \pm 7,2)$ \\
\hline \multicolumn{2}{|l|}{ ICU admission principal diagnosis (\%) } \\
\hline - Renal & $15 / 30(50)$ \\
\hline - Septic shock & $7 / 30(23)$ \\
\hline - Respiratory & $5 / 30(17)$ \\
\hline - Intoxication & $2 / 30(7)$ \\
\hline - Neurological & $1 / 30(3)$ \\
\hline APACHE II score & $21( \pm 6)$ \\
\hline SOFA Score (median, range) & $7(2-16)$ \\
\hline \multicolumn{2}{|l|}{ Surgical status at admission (\%) } \\
\hline 1- Urgent & $3 / 30(10)$ \\
\hline 2- Non-surgical & $27 / 30(90)$ \\
\hline \multicolumn{2}{|l|}{ Renal variables } \\
\hline - sCr at AKI diagnosis (mg/dL) & $5.3(3.5)$ \\
\hline - BUN at AKI diagnosis (mg/dL) & $191(118)$ \\
\hline - RRT during ICU stay & $22 / 30(73)$ \\
\hline
\end{tabular}


Table 1. Continued

\begin{tabular}{ll} 
Variables & Values \\
\hline Chronic comorbidities (\%) & \\
- Hypertension & $18 / 30(60)$ \\
- Diabetes & $13 / 30(43)$ \\
- COPD & $3 / 30(10)$ \\
- Coronary artery disease & $5 / 30(17)$ \\
- Heart failure & $9 / 30(30)$ \\
- Peripheral vascular disease & $12 / 30(40)$ \\
- Immunocompromised & $4 / 30(13)$ \\
- Chronic liver disease & $4 / 30(13)$ \\
- Malignancy & $1 / 30(3)$ \\
- Chronic kidney disease (no dialysis) & $17 / 30(57)$ \\
\hline Acute comorbidities at ICU admission (first 24h) (\%) & \\
- Acute kidney injury & $25 / 30(83)$ \\
- Septic status & $8 / 30(27)$ \\
- Renal replacement therapy & $18 / 30(60)$ \\
- Oliguria & $20 / 30(67)$ \\
- Vasoactive drugs & $9 / 30(30)$
\end{tabular}

${ }^{*}$ Available in 18 patients. BMI, Body mass index; BUN, Blood urea nitrogen; COPD, Chronic obstructive pulmonary disease; ICU, Intensive care unit; RRT, Renal replacement therapy; sCr, serum creatinine. Continuous variables are expressed as mean (standard deviation) unless otherwise specified.

Table 2 reports the average muscle thickness of each measurement. Bivariate analysis (Figure 1) showed that, as expected, US and CT yielded identical values of muscle thickness comparing left to right side $(-0.03 \mathrm{~cm}(\mathrm{P}=0.32)$, and $-0.018(\mathrm{P}=0.50)$, for US and $C T$, respectively).

Table 2. Pairwise comparison between Ultrasound and CT scan measurements

\begin{tabular}{lll} 
Variable & Value obtained using US $(\mathbf{c m})$ & Value obtained using $\mathbf{C T}(\mathbf{c m})$ \\
\hline RF r Prox & $1.58(0.33)$ & $1.60(0.41)$ \\
\hline VI r Prox & $1.19(0.43)$ & $1.16(0.44)$ \\
\hline RF r Dist & $1.13(0.36)$ & $1.12(0.35)$ \\
\hline VI r Dist & $1.02(0.39)$ & $0.98(0.42)$ \\
\hline RF I Prox & $1.57(0.35)$ & $1.59(0.38)$ \\
\hline VI I Prox & $1.12(0.39)$ & $1.13(0.39)$ \\
\hline RF I Dist & $1.15(0.37)$ & $1.13(0.35)$ \\
\hline VI I Dist & $0.97(0.39)$ & $0.95(0.39)$
\end{tabular}

CT, computerized tomography; Dist, distal; I, left; RF, rectus femoris; Prox, proximal; r, right; US, ultrasound; VI Vastus intermedius.

In contrast, in both US and CT scans, VI differed from RF, and Distal differed from Proximal measurement by approximately $-0.3 \mathrm{~cm}(\mathrm{P}<0.001$ for the comparison between $\mathrm{VI}$ vs $\mathrm{RF}$ and between Proximal vs Distal, both in US and CT scans; Figure 1). The estimated SD of 
measurement error was approximately $0.2 \mathrm{~cm}$ for both techniques, although it was numerically slightly larger for US compared to CT (Figure 1). The overall standard deviation of betweenindividual differences was approximately $0.35 \mathrm{~cm}$ (Figure 1).

Figure 1. Schematic representation of the bivariate mixed model fitted on joint CT and US data to estimate the differences in muscle thickness between muscle types (VI vs RF), different positions (distal vs proximal) and different sides (left vs right).

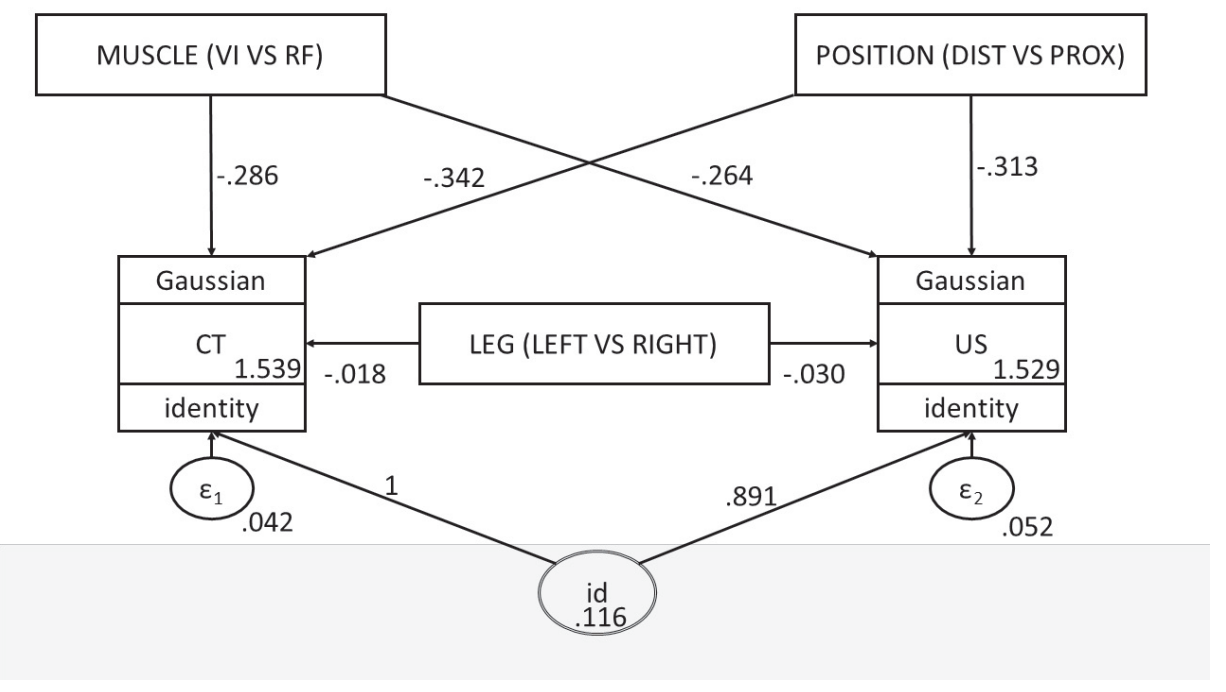

There was no difference in muscle thickness between the left and right side for both CT $(-0.018 \mathrm{~cm}(P=0.50))$ and US $(-0.03 \mathrm{~cm}(P=0.32))$. On the other hand, VI muscle thickness was lower compared to RF $(-0.286 \mathrm{~cm}$ for $\mathrm{CT}(\mathrm{P}<0.001)$ and -0.264 for $\mathrm{US}(\mathrm{P}<0.001))$ and Distal muscle thickness was lower compared to Proximal thickness $(-0.34 \mathrm{~cm}$ for $C T(P<0.001)$ and $-0.313 \mathrm{C}$, for US $(P<0.001))$. The variance of measurement error was $0.042 \mathrm{~cm}$, and $0.052 \mathrm{~cm}$ for $C T$ and US, respectively (the standard deviations, which are obtained by taking the square root of the variance, were $0.20 \mathrm{~cm}$, and 0.23 , respectively; $\mathrm{P}=0.14$ ). The between-subject variance was $0.12 \mathrm{~cm}$ (the standard deviation was $0.35 \mathrm{~cm}$ ).

The expressions "Gaussian" and "Identity" in the square boxes indicate that the dependent variables CT and US were analyzed as normally distributed variables (i.e. "Gaussian"), and that the regression model was an ordinary linear regression model on its natural scale (i.e. "Identity"), in cm. Rectangles represent the independent variables, whereas circles represent errors. According to the model there are two kinds of error (i.e. two causes of random variation about the population average at each measurement site) namely, measurement error (i.e. intra-patient variability), which is represented by the circle containing the letter " $\varepsilon$ " and, error due to inter-patient variability, which is represented by the circle containing ("id"); the latter is drawn under a gray-shaded stripe to indicate that this error is shared by all measurements taken from the same patient. Arrows represent what causes a given CT or US measurement take 
its specific observed value. Number along arrows represent coefficients, whereas numbers close to circles represent the variance of the error, and in the square box the overall mean in the reference category (i.e. proximal right rectus femoralis).

For instance, for a given patient, the CT distal vastus intermedius measurement is equal to $1.539 \mathrm{~cm}$, minus $0.286 \mathrm{~cm}$ (because the site is vastus intermedius instead of retus femoralis), minus $0.342 \mathrm{~cm}$ (because the site it is distal instead of proximal), plus/minus the measurement error $\varepsilon 1$ in $\mathrm{cm}$, plus/minus the extent in $\mathrm{cm}$ the patient differed from average value.

$\mathrm{CT}$, Computer tomography scan; US, Ultrasound scan; VI, vastus intermedius muscle; RF, rectus femoralis muscle; Prox, proximal measurement; Dist measurement $\varepsilon 1$, variance (i.e. standard deviation squared) of CT measurement error; $\varepsilon 2$, variance (i.e. standard deviation squared) of US measurement error; id between-subject variance (i.e. standard deviation squared) in muscle thickness.

Figure 2 (panel A-D) and Table 3 shows the bias analysis comparing US and CT. When comparing US to $\mathrm{CT}$, both the observed differential bias (between +0.04 and $+0.26 \mathrm{~cm}$, depending on the muscle site) and the proportional bias (between $82 \%$ and $98 \%$ of the reference value, depending on the muscle site) were not statistically significant. Besides statistical significance the point estimates of the differential bias and proportional bias of US VS CT were remarkably close to the null value (i.e. $0 \mathrm{~cm}$, and $100 \%$, respectively), with the possible exception of the RF, Proximal (Figure 2 panel A, and Table 3). 
Figure 2. Bias plots showing bias comparing US (blue dots and fitted line) vs CT (brown dots and fitted line).
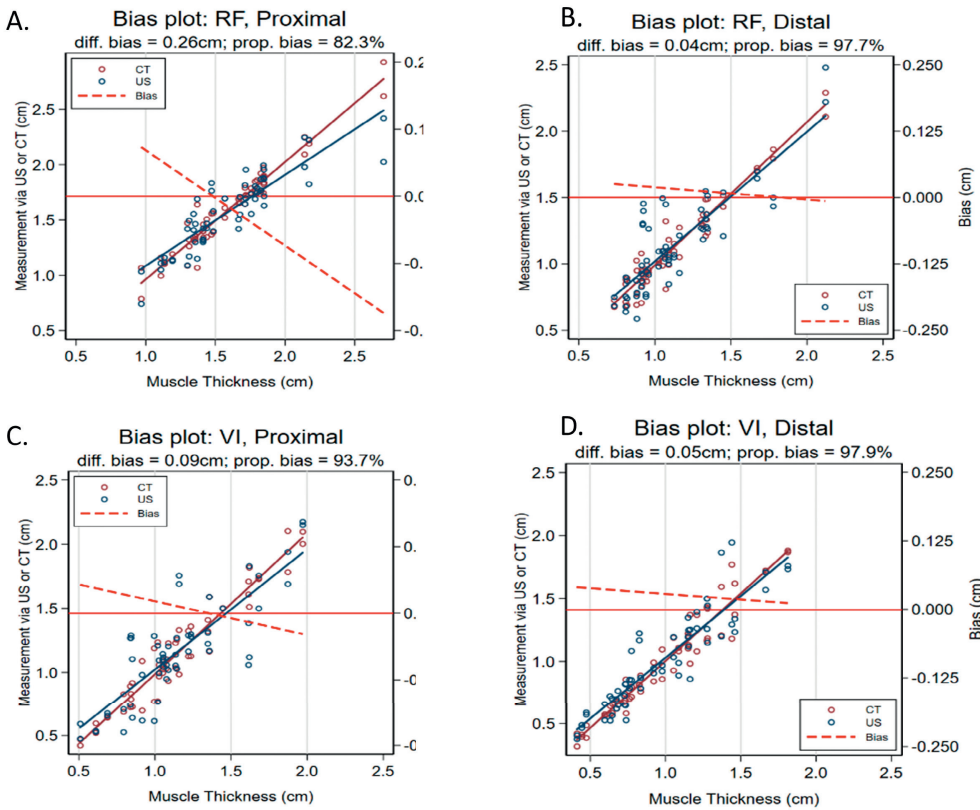

D. Bias plot: VI, Distal

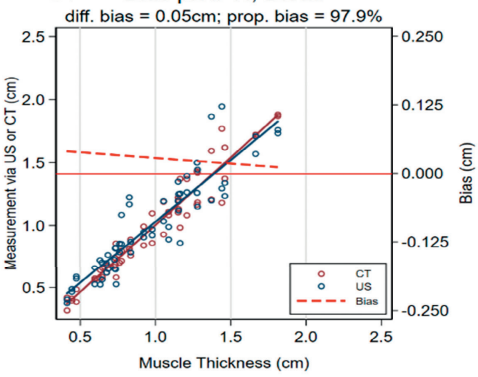

The left $y$-axis shows the US and CT measures (in $\mathrm{cm}$ ), whereas the right $y$-axis shows the bias $(\mathrm{cm})$. The $x$-axis reports the true (latent) value of muscle thickness. The dotted red line refers to the bias, which has to be compared to the horizontal red line representing the ideal line of complete absence of bias. The bias changes linearly as a function of the true (latent) value of muscle thickness. The subtitle on the top reports the bias as absolute difference in $\mathrm{cm}$ (differential bias) and relative difference in percentage (proportional bias). Numerically, compared to CT, on US scan RF, Prox was on average $+0.26 \mathrm{~cm}$ thicker (differential bias), although the percentage difference of US vs CT was $83 \%$, (proportional bias) implying that the bias tended to change with larger absolute values of muscle thickness: the larger the muscle thickness the less positive the bias.

$\mathrm{CT}$, Computer tomography scan; US, Ultrasound scan; VI, vastus intermedius muscle; RF, rectus femoralis muscle; Prox, proximal measurement; Dist measurement. 
Table 3. Estimated bias comparing US vs CT

\begin{tabular}{lll} 
& $\begin{array}{l}\text { Differential Bias }- \text { cm } \\
\text { (95\% confidence interval) } \\
\text { comparing US vs CT }\end{array}$ & $\begin{array}{l}\text { Proportional Bias }-\% \\
\text { (95\% confidence interval) } \\
\text { comparing US vs CT }\end{array}$ \\
\hline RF, Prox & $+0.26(-0.03$ to +0.56$)$ & $82.3 \%(63.2$ to 100.1$)$ \\
\hline RF, Dist & $+0.04(-0.24$ to 0.33$)$ & $97.7 \%(72.5$ to 122.9$)$ \\
\hline VI, Prox & $+0.09(-0.13$ to +30.6$)$ & $93.7 \%(74.6$ to 112.8$)$ \\
\hline VI, Dist & $+0.05(-0.04$ to +0.14$)$ & $97.9 \%(88.0$ to 107.8$)$
\end{tabular}

Bias comparing US vs CT as absolute difference in $\mathrm{cm}$ (differential bias) and relative difference in percentage (proportional bias). A 95\% confidence interval of the differential bias including the value of zero implies non-statistical significance; a 95\% confidence interval of the proportional bias including the value of 100 implies non statistical significance. Numerically, compared to $\mathrm{CT}$, on US scan RF, Prox was on average $+0.26 \mathrm{~cm}$ thicker (differential bias), although the percentage difference of US vs CT was $83 \%$, (proportional bias) implying that the bias tended to change with larger absolute values of muscle thickness: the larger the muscle thickness: the less positive the difference between US and CT. However, the $95 \%$ of the confidence interval of the differential bias of $+0.26 \mathrm{~cm}$ included zero, and the $95 \%$ confidence interval of the proportional bias included 100, therefore US and CT were not significantly different.

$\mathrm{CT}$, Computer tomography scan; US, Ultrasound scan; VI, vastus intermedius muscle; RF, rectus femoralis muscle; Prox, proximal measurement; Dist measurement

Figure 3 (panel A-D) reports the precision plots, showing that, confirming the finding reported above, US scan tended to be a slightly less precise technique compare to $\mathrm{CT}$, over all the range of values of muscle thickness. 
Figure 3. Precision plots showing precision comparing US (blue circles) vs CT (brown circles).

A.

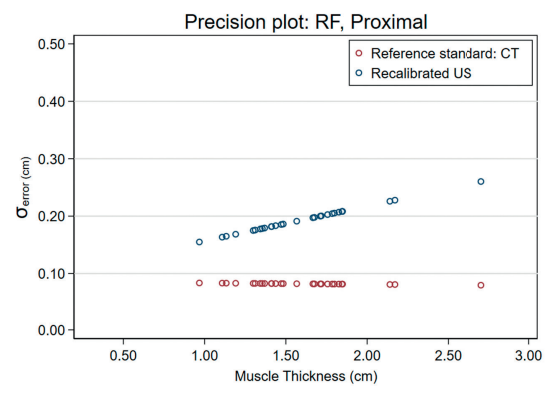

C.

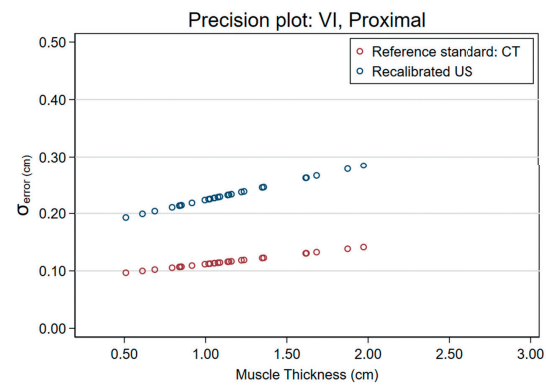

B.

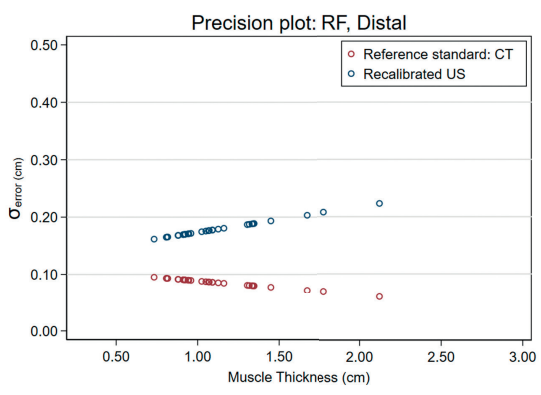

D.

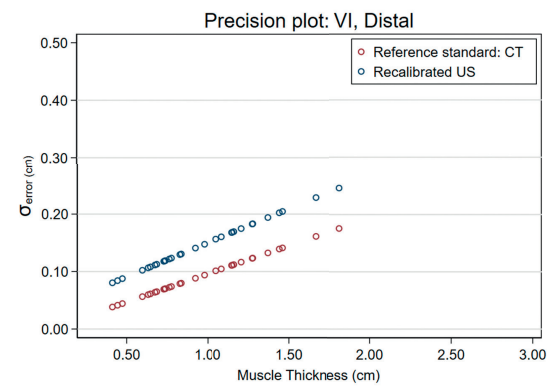

The $y$-axis represents precision, which is displaied as the standard deviation $\sigma$ (i.e. the square root of the variance) of the measurement error in $\mathrm{cm}$. The $\mathrm{x}$-axis reports the true (latent) value of muscle thickness. Compared to CT (brown circles), US (blue circles) tended to be slightly less precise (i.e to have a larger values of $\sigma$ ) over the entire range of values of muscle thickness for any measurement (RF, VI, proximal and distal).

CT, Computer tomography scan; US, Ultrasound scan; VI, vastus intermedius muscle; RF, rectus femoralis muscle; Prox, proximal measurement; Dist measurement.

Since the differential and proportional bias estimates were similar between VI, RF, Proximal and Distal, and the measurement error was anyhow close between US and CT and it was also approximately constant over all the range of muscle thickness values, we pooled all the data to draw a Bland-Altman plot with 95\% limits of agreement (Figure 4) which were between -0.34 and $+0.36 \mathrm{~cm}$. 
Figure 4. Bland-Altman plot showing $95 \%$ limits of agreement between US and CT with all data pooled together.

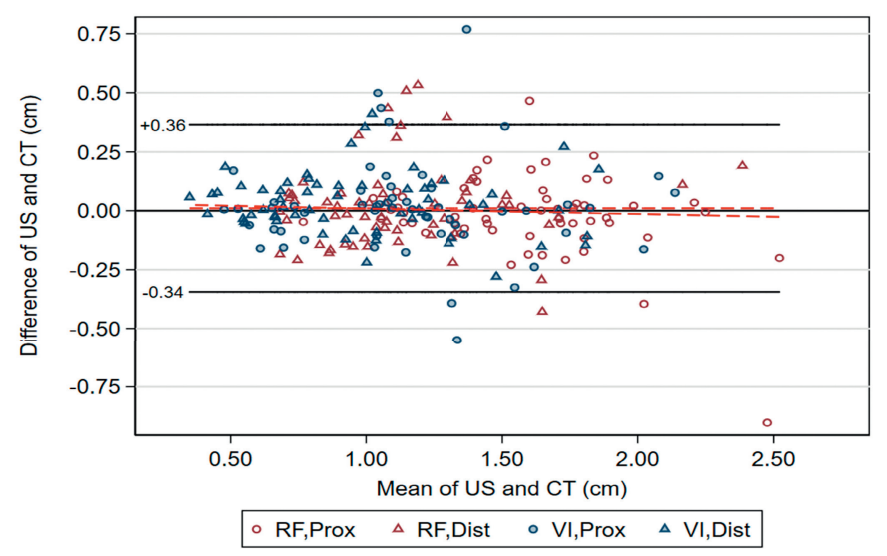

The $y$-axis represents the difference between US and CT, the $x$-axis their average. The solid horizontal black lines represent the $95 \%$ limits of agreement, which were -0.34 and $+0.36 \mathrm{~cm}$, respectively. The dotted red lines represent the (negligible) bias, both as a constant value (horizontal line, analogous to the differential bias shown above) and as a linear function of the mean (line with declining slope, analogous to the proportional bias shown above).

$\mathrm{CT}$, Computer tomography scan; US, Ultrasound scan; VI, vastus intermedius muscle; RF, rectus femoralis muscle; Prox, proximal measurement; Dist measurement.

\section{DISCUSSION}

In the present study, we newly report how the US technique compares to CT scan for the measurement of quadriceps muscle thickness of critically ill patients with AKI. Our study provides evidence that, compared to CT scan, US bias is negligible for most of the measurements, and its precision is close to that of CT scan.

Data are in accordance to other studies validating the US technique for the assessment of quadriceps muscle mass in clinical settings different from the ICU $(24,25)$. In one study in patients with coronary artery disease (CAD), rectus femoris thickness of 20 patients was measured by US and compared to CT scans (24). A high correlation between measurements with low bias and narrow limits of agreement was found. In another study in patients with chronic obstructive pulmonary disease (COPD), rectus femoris cross-sectional area (RFCSA) assessed by US was compared to the whole quadriceps cross-sectional area (QCSA) assessed by CT (25). A high intra-class correlation coefficient (ICC $=0.88$ ), with non-significant bias, was found. Recently, muscle US measurements have been validated against CT scan also in patients 
with chronic kidney disease (26). Similarly, studies comparing muscle mass measurement obtained by MRI, another gold standard technique, and US found no difference between the different methods, again confirming very high correlation coefficients and agreement in young and elderly healthy subjects (27-29). In a study comparing quadriceps thickness measured by US and DEXA in COPD patients, US was found to have good reproducibility, and to be more sensitive to changes in muscle mass when compared to DEXA (30).

To our knowledge, this is the first study evaluating the validity of quadriceps muscle thickness assessment by US against a standard reference method in a cohort of critically ill patients. Earlier studies in critically ill patients compared US with muscle biopsies (1), or with muscle strength, as assessed using the Medical Research Council score (MRC-SS), or with muscle function, as assessed using the physical function in intensive care test score (PFIT-s) and the ICU mobility scale (IMS) (31). In these studies, muscle US was able to detect muscle loss (1), and to predict muscle strength and function (31).

Muscle US has been shown to be sensitive enough to detect even small changes in muscle mass during the first 10 days of ICU stay $(1,31,32)$. In addition, muscle wasting, as assessed by bedside US, was able to predict adverse outcomes in surgical ICU patients (33). In the critical care setting, the stratification of patients at risk of muscle wasting is essential to allow the optimization of the clinical and therapeutic management aimed at preventing muscle loss, and muscle US could represent a very useful screening tool in this regard (34). A recent review analyzed 7 studies for a total of 330 patients admitted to the ICU for at least 7 days, suffering from sepsis and multi-organ failure, in which the Authors used US to evaluate muscle thickness or cross-sectional area at the level of the arm, forearm and thigh (14). Muscle thickness at ICU admission was significantly decreased compared to healthy controls. In addition, decreased quadriceps muscle size as measured by US was an independent risk factor for unscheduled readmission or death in another study (35). Thus, muscle US could represent in the future an important tool for both nutritional screening and prognostic assessment.

One additional strength of our study is that it provides US methodology estimates that can be used to develop and implement new protocols. Besides quantifying measurement error (standard deviation of $0.2 \mathrm{~cm}$ ), we demonstrated that in a relatively old non-athletic population there is no difference between quadriceps muscle thicknesses in both legs, as expected, that the proximal measurements were thicker than the distal measurements and that the rectus femoris muscle was thicker than the vastus intermedius by about $0.3 \mathrm{~cm}$. However, in the bias analysis, we noticed that the rectus femoris proximal measurement (RF, Prox) had the largest measurement error. Despite its non-statistical significance, it is important to notice that this repere tended to be the largest, suggesting a possible source of error in untrained assessors that might put more pressure on the probe to visualize the whole muscle. To allow for an accurate image and measurement is very important to use excess contact gel between the probe and the thigh, in order to put as little pressure as possible. Overall, the US took less 
than 10 minutes to set up and complete image acquisition and less than 10 minutes per image to complete measurement analysis.

It is important to address the limitations of our study, as well as the possible limitations for the use of US for the assessment of muscle mass. First of all, due to the limited number of patients enrolled we could not explore the prognostic value of quadriceps muscle thickness in the specific population of critically ill patients with AKI. Nevertheless, as reported above, a recent study in the ICU setting suggests that reduced muscle mass as assessed by US may predict adverse outcomes (33). Secondly, the assessment of muscle thickness by US may be operator dependent. In our study, only one experienced assessor was responsible for all the US measurements. However, a reliability study published by our group on patients in the same clinical setting found high intraclass correlation coefficients (ICC) between non experienced operators that have received formal training and followed a standardized protocol in order to obtain US images and measuring muscle thickness (15).

In conclusion, US is a simple, easily applicable, valid, accurate and reliable method for skeletal muscle evaluation in critically ill patients with AKI. In these patients, quadriceps muscle thickness assessed by US is consistent with CT measures, and could have value both in the clinical practice of nutritional support, as well, as potentially, for risk stratification.

Further studies aimed at defining cut-off values for normal muscle mass are needed, in order to allow the early identification of patients with low muscle mass at ICU admission.

Practical application: Quadriceps muscle ultrasound is a reliable and valid method for bedside muscle mass evaluation in critically ill patients with AKI. In addition, the present paper provides practical information for the implementation of US-based protocols for muscle mass assessment. 


\section{STATEMENT OF AUTHORSHIP}

All persons who meet authorship criteria are listed as authors, and all authors certify that they have participated sufficiently in the work to take public responsibility for the content, including participation in the concept, design, analysis, writing, or revision of the manuscript.

Ethical approval: All procedures performed in studies involving human participants were in accordance with the ethical standards of the regional research committee AVEN (authorization 9713 of March 14, 2017), and with the 1964 Helsinki Declaration and its later amendments or comparable ethical standards.

Conflict of Interest: The authors declare that they have no conflict of interest.

Support and financial disclosure: This work was supported in part by the Italian Society of Artificial Nutrition and Metabolism (Società Italiana di Nutrizione Artificiale e Metabolismo, SINPE). The authors declare that they have no conflict of interest. 


\section{REFERENCES OF CHAPTER 3}

1. Puthucheary ZA, Rawal J, McPhail M, Connolly B, Ratnayake G, Chan P, Hopkinson NS, Phadke R, Dew T, Sidhu PS, Velloso C, Seymour J, Agley CC, Selby A, Limb M, Edwards LM, Smith K, Rowlerson A, Rennie MJ, Moxham J, Harridge SD, Hart N, Montgomery HE (2013) Acute skeletal muscle wasting in critical illness. Jama 310 (15):1591-1600. doi:10.1001/jama.2013.278481

2. Koukourikos K, Tsaloglidou A, Kourkouta L (2014) Muscle atrophy in intensive care unit patients. Acta informatica medica. Acta Inform Med 22 (6):406-410. doi:10.5455/aim.2014.22.406-410

3. Singer P, Blaser AR, Berger MM, Alhazzani W, Calder PC, Casaer MP, Hiesmayr M, Mayer K, Montejo JC, Pichard C, Preiser JC, van Zanten ARH, Oczkowski S, Szczeklik W, Bischoff SC (2019) ESPEN guideline on clinical nutrition in the intensive care unit. Clin Nutr 38 (1):48-79. doi:10.1016/j.clnu.2018.08.037

4. Puthucheary Z, Montgomery H, Moxham J, Harridge S, Hart N (2010) Structure to function: muscle failure in critically ill patients. J Physiol 588 (23):4641-4648. doi:10.1113/jphysiol.2010.197632

5. NA A, O’Brien JM, Jr., Hoffmann SP, Phillips G, Garland A, Finley JC, Almoosa K, Hejal R, Wolf KM, Lemeshow S, Connors AF, Jr., Marsh CB (2008) Acquired weakness, handgrip strength, and mortality in critically ill patients. Am J Respir Crit Care Med 178 (3):261-268. doi:10.1164/rccm.200712-18290C

6. Hiesmayr M (2012) Nutrition risk assessment in the ICU. Curr Opin Clin Nutr Metab Care 15 (2):174180. doi:10.1097/MCO.0b013e328350767e

7. Wieske L, Dettling-Ihnenfeldt DS, Verhamme C, Nollet F, van Schaik IN, Schultz MJ, Horn J, van der Schaaf M (2015) Impact of ICU-acquired weakness on post-ICU physical functioning: a follow-up study. Crit Care 19:196. doi:10.1186/s13054-015-0937-2

8. Puthucheary ZA, Hart N (2014) Skeletal muscle mass and mortality - but what about functional outcome? Crit Care 18 (1):110. doi:10.1186/cc13729

9. Weijs PJ, Looijaard WG, Dekker IM, Stapel SN, Girbes AR, Oudemans-van Straaten HM, Beishuizen A (2014) Low skeletal muscle area is a risk factor for mortality in mechanically ventilated critically ill patients. Crit Care 18 (2):R12. doi:10.1186/cc13189

10. Fiaccadori E, Regolisti G, Maggiore U (2013) Specialized nutritional support interventions in critically ill patients on renal replacement therapy. Curr Opin Clin Nutr Metab Care 16 (2):217-224. doi:10.1097/ MCO.0b013e32835c20b0

11. Sykes L, Nipah R, Kalra P, Green D (2018) A narrative review of the impact of interventions in acute kidney injury. J Nephrol 31 (4):523-535. doi:10.1007/s40620-017-0454-2

12. Fani F, Regolisti G, Delsante M, Cantaluppi V, Castellano G, Gesualdo L, Villa G, Fiaccadori E (2018) Recent advances in the pathogenetic mechanisms of sepsis-associated acute kidney injury. J Nephrol 31 (3):351-359. doi:10.1007/s40620-017-0452-4 
13. Buckinx F, Landi F, Cesari M, Fielding RA, Visser M, Engelke K, Maggi S, Dennison E, Al-Daghri NM, Allepaerts S, Bauer J, Bautmans I, Brandi ML, Bruyere O, Cederholm T, Cerreta F, Cherubini A, Cooper C, Cruz-Jentoft A, McCloskey E, Dawson-Hughes B, Kaufman JM, Laslop A, Petermans J, Reginster JY, Rizzoli R, Robinson S, Rolland Y, Rueda R, Vellas B, Kanis JA (2018) Pitfalls in the measurement of muscle mass: a need for a reference standard. J Cachexia Sarcopenia Muscle 9 (2):269-278. doi:10.1002/jcsm. 12268

14. Connolly B, MacBean V, Crowley C, Lunt A, Moxham J, Rafferty GF, Hart N (2015) Ultrasound for the assessment of peripheral skeletal muscle architecture in critical illness: a systematic review. Crit Care Med 43 (4):897-905. doi:10.1097/CCM.0000000000000821

15. Sabatino A, Regolisti G, Bozzoli L, Fani F, Antoniotti R, Maggiore U, Fiaccadori E (2017) Reliability of bedside ultrasound for measurement of quadriceps muscle thickness in critically ill patients with acute kidney injury. Clin Nutr 36 (6):1710-1715. doi:10.1016/j.clnu.2016.09.029

16. Sabatino A, Regolisti G, Delsante M, Di Motta T, Cantarelli C, Pioli S, Grassi G, Batini V, Gregorini M, Fiaccadori E (2019) Noninvasive evaluation of muscle mass by ultrasonography of quadriceps femoris muscle in End-Stage Renal Disease patients on hemodialysis. Clin Nutr 38 (3):1232-1239. doi:10.1016/j.clnu.2018.05.004

17. Kidney Disease: Improvig Global Outcomes (2012) KDIGO Clinical Practice Guideline for Acute Kidney Injury. Kidney Int Suppl 2:1 - 138

18. Bossuyt PM, Reitsma JB, Bruns DE, Gatsonis CA, Glasziou PP, Irwig L, Lijmer JG, Moher D, Rennie D, de Vet HC, Kressel HY, Rifai N, Golub RM, Altman DG, Hooft L, Korevaar DA, Cohen JF, Group S (2015) STARD 2015: an updated list of essential items for reporting diagnostic accuracy studies. BMJ 351:h5527. doi:10.1136/bmj.h5527

19. Tillquist M, Kutsogiannis DJ, Wischmeyer PE, Kummerlen C, Leung R, Stollery D, Karvellas CJ, Preiser JC, Bird N, Kozar R, Heyland DK (2014) Bedside ultrasound is a practical and reliable measurement tool for assessing quadriceps muscle layer thickness. JPEN J Parenter Enteral Nutr 38 (7):886-890. doi:10.1177/0148607113501327

20. Bland JM, Altman DG (1986) Statistical methods for assessing agreement between two methods of clinical measurement. Lancet (London, England) 1 (8476):307-310

21. Taffe $P$ (2018) Effective plots to assess bias and precision in method comparison studies. Statistical methods in medical research 27 (6):1650-1660. doi:10.1177/0962280216666667

22. Taffé P, Peng M, Stagg V, Williamson T (2017) biasplot: A package to effective plots to assess bias and precision in method comparison studies. Stata Journal 17 (1):208-221

23. Bland JM, Altman DG (2007) Agreement between methods of measurement with multiple observations per individual. J Biopharm Stat 17 (4):571-582. doi:10.1080/10543400701329422

24. Thomaes T, Thomis M, Onkelinx S, Coudyzer W, Cornelissen V, Vanhees L (2012) Reliability and validity of the ultrasound technique to measure the rectus femoris muscle diameter in older CAD-patients. BMC Med Imaging 12:7. doi:10.1186/1471-2342-12-7

25. Seymour JM, Ward K, Sidhu PS, Puthucheary Z, Steier J, Jolley CJ, Rafferty G, Polkey MI, Moxham J (2009) Ultrasound measurement of rectus femoris cross-sectional area and the relationship with quadriceps strength in COPD. Thorax 64 (5):418-423. doi:10.1136/thx.2008.103986 
26. Souza VA, Oliveira D, Cupolilo EN, Miranda CS, Colugnati FAB, Mansur HN, Fernandes N, Bastos MG (2018) Rectus femoris muscle mass evaluation by ultrasound: facilitating sarcopenia diagnosis in pre-dialysis chronic kidney disease stages. Clinics (Sao Paulo, Brazil) 73:e392. doi:10.6061/clinics/2018/e392

27. Bemben MG (2002) Use of diagnosticultrasound for assessing musclesize. J Strength Cond Res 16 (1):103-108

28. Reeves ND, Maganaris CN, Narici MV (2004) Ultrasonographic assessment of human skeletal muscle size. Eur J Appl Physiol. 91 (1):116-118. doi:10.1007/s00421-003-0961-9

29. Arbeille P, Kerbeci P, Capri A, Dannaud C, Trappe SW, Trappe TA (2009) Quantification of muscle volume by echography: comparison with MRI data on subjects in long-term bed rest. Ultrasound Med Biol 35 (7):1092-1097. doi:10.1016/j.ultrasmedbio.2009.01.004

30. Menon MK, Houchen L, Harrison S, Singh SJ, Morgan MD, Steiner MC (2012) Ultrasound assessment of lower limb muscle mass in response to resistance training in COPD. Respir Res 13:119. doi:10.1186/1465-9921-13-119

31. Parry SM, El-Ansary D, Cartwright MS, Sarwal A, Berney S, Koopman R, Annoni R, Puthucheary Z, Gordon IR, Morris PE, Denehy L (2015) Ultrasonography in the intensive care setting can be used to detect changes in the quality and quantity of muscle and is related to muscle strength and function. J Crit Care 30 (5):1151 e1159-1114. doi:10.1016/j.jcrc.2015.05.024

32. Segers J, Hermans G, Charususin N, Fivez T, Vanhorebeek I, Van den Berghe G, Gosselink R (2015) Assessment of quadriceps muscle mass with ultrasound in critically ill patients: intra- and inter-observer agreement and sensitivity. Intensive Care Med 41 (3):562-563. doi:10.1007/s00134-015-3668-6

33. Mueller N, Murthy S, Tainter CR, Lee J, Riddell K, Fintelmann FJ, Grabitz SD, Timm FP, Levi B, Kurth T, Eikermann M (2016) Can Sarcopenia Quantified by Ultrasound of the Rectus Femoris Muscle Predict Adverse Outcome of Surgical Intensive Care Unit Patients as well as Frailty? A Prospective, Observational Cohort Study. Ann Surg 264 (6):1116-1124. doi:10.1097/SLA.0000000000001546

34. Landi F, Camprubi-Robles M, Bear DE, Cederholm T, Malafarina V, Welch AA, Cruz-Jentoft AJ (2018) Muscle loss: The new malnutrition challenge in clinical practice. Clin Nutr. doi:10.1016/j. clnu.2018.11.021

35. Greening NJ, Harvey-Dunstan TC, Chaplin EJ, Vincent EE, Morgan MD, Singh SJ, Steiner MC (2015) Bedside assessment of quadriceps muscle by ultrasound after admission for acute exacerbations of chronic respiratory disease. Am J Respir Crit Care Med 192 (7):810-816. doi:10.1164/rccm.201503-05350C 



\section{CHAPTER 4}

Noninvasive evaluation of muscle mass by ultrasonography of quadriceps femoris muscle in End-Stage Renal Disease patients on hemodialysis

Sabatino A, Regolisti G, Delsante M, Di Motta T, Cantarel-

li C, Pioli S, Grassi G, Batini V, Gregorini M, Fiaccadori E 


\section{ABSTRACT}

Background and Aims: Protein-Energy Wasting (PEW) is a pathological condition of renal patients with advanced Chronic Kidney Disease characterized by a progressive reduction of energy and protein assets. The assessment of nutritional status, especially for what concerns muscle mass, is essential for the identification of patients at risk for the development of PEW, as well as monitoring the effects of nutritional interventions. Ultrasound methods are easily applicable at the bedside for quantitative assessment of skeletal muscle.

The present study was aimed at evaluating quadriceps rectus femoris thickness (QRFT) and quadriceps vastus intermedius thickness (QVIT) in patients on chronic hemodialysis. .

Methods: This was a prospective observational study. Three groups of adult patients were studied: young healthy subjects, well-nourished hospitalized patients with normal renal function, and End-Stage Renal Disease patients on hemodialysis (ESRD-HD). QRFT and QVIT were measured at two sites bilaterally (8 measures/patient) and were compared between groups, and also between subgroups of ESRD-HD patients stratified on the basis of conventional nutritional status parameters.

Results: We enrolled 35 healthy subjects, 30 hospitalized patients, and 121 ESRD patients on hemodialysis. QRFT and QVIT of ESRD patients on hemodialysis were lower than those of both control groups $(P<0.001)$. After stratifying ESRD patients into subgroups based on nutritional variable cut-offs commonly used to define PEW in this clinical setting (BMI $(\geq 23$ versus $<23$ $\mathrm{Kg} / \mathrm{m} 2$ ), albumin ( $\geq 3.8$ versus $<3.8 \mathrm{~g} / \mathrm{dL}$ )) and malnutrition inflammation score (MIS) status (< 6 versus $\geq 6$ ), QRFT and QVIT of patients with worse nutritional status were significantly lower than those of well-nourished ESRD-HD patients ( $P$ value range: $<0.001-<0.05$ ).

Conclusion: Skeletal muscle ultrasound is a simple and easily applicable bedside technique in the dialysis units, and could represent an adequate tool for the identification of patients with reduced muscle mass.

Key words: hemodialysis, nutritional status evaluation, protein energy wasting, skeletal muscle mass, ultrasound. 


\section{INTRODUCTION}

End-Stage Renal Disease on chronic hemodialysis (ESRD-HD) is characterized by progressive worsening of nutritional status with a high prevalence of Protein-Energy Wasting (PEW) (1), a pathological condition associated with poor outcome (1). Thus, the assessment of nutritional status is essential both for preventing PEW in patients at risk, and for monitoring nutritional support in those with established PEW (2).

In this regard, increasing attention is devoted to the assessment of muscle mass. Skeletal muscle is in fact the largest store of Lean Body Mass (LBM) protein, and its quantitative/ qualitative alterations may impact negatively on patients' quality of life and prognosis (3). In ESRD-HD patients muscle mass is usually assessed by bedside techniques, such as anthropometry, bioimpedance spectroscopy (BIS), or bioimpedance analysis (BIA), which however have some limitations (3). As a matter of fact, the gold standard imaging techniques such as computerized tomography (CT), magnetic resonance imaging (MRI), and Dual Energy X-ray Absorptiometry (DEXA) are expensive, not available at the bedside, and finally imply exposure to radiation or strong magnetic fields. Conversely, ultrasonography (US) is commonly available in many different clinical settings, can be applied to the evaluation of different skeletal muscle groups, and allows bedside serial measurements of muscle cross-sectional diameter and area $(4,5)$. Thus, this noninvasive method could represent a good alternative to more sophisticated and expensive techniques. Ultrasonographic measurements of the quadriceps muscle (the single largest skeletal muscle group in the body) are highly correlated to muscle mass as assessed by gold standard methods $(6,7)$. Specifically, quadriceps femoris muscle thickness best correlates with fat-free mass by DEXA (6), and appears to be as accurate as the quantification of muscle mass by CT or MRI $(7,8)$. Finally, quadriceps femoris US has been suggested recently as a reliable and easily reproducible method to assess muscle thickness in patients with Acute Kidney Injury (AKI) (9). The same study also showed that even the rapid fluid shifts caused by renal replacement therapy in edematous patients did not affect US measurements of quadriceps muscle thickness (9).

No data are currently available on the utilization of quadriceps femoris muscle US in patients with ESRD-HDs. Thus, in the present study we performed US measurements to assess the thickness of two out of four muscles constituting the quadriceps femoris (namely, the rectus femoris (quadriceps rectus femoris thickness, QRFT) and the vastus intermedius (quadriceps vastus intermedius thickness, QVIT)) in patients with ESRD-HD. Healthy subjects and sedentary hospitalized patients with normal renal function and nutritional status were used as controls.

The specific aims of the study were:

a) to evaluate if QRFT and QVIT are decreased in patients with ESRD-HD in comparison to both healthy subjects and well-nourished hospitalized patients with normal renal function;

b) to evaluate if QRFT and QVIT are different in patients with ESRD-HD when they are stratified based on conventional diagnostic criteria for PEW; 
c) to ascertain if a correlation exists between US measures and conventional variables utilized for the evaluation of muscle mass and nutritional status in the same clinical setting;

d) to ascertain if measurements in ESRD-HD are affected by the dialysis procedure itself.

\section{MATERIALS AND METHODS}

This was a cross-sectional, prospective observational study performed in Italy at six Hemodialysis Centers of the Italian National Health System (Parma, Borgo Val di Taro, Livorno, Piombino, Cecina and Reggio Emilia). The study was approved by the local Institutional Review Boards (Ref. $n^{\circ}$ 45737, December 12th, 2015). The procedures were in agreement with the Declaration of Helsinki, and written informed consent was obtained from all participants.

The following groups of subjects were enrolled:

A - Healthy subjects: We enrolled 35 subjects from the hospital staff. Inclusion criteria were BMI $>18.5 \mathrm{Kg} / \mathrm{m} 2$, Subjective Global Assessment (SGA) class A, absence of chronic or acute illnesses.

B - Hospitalized patients with normal renal function: We enrolled 30 hospitalized adult patients with normal renal function from internal medicine wards. Inclusion criteria were BMI > 18.5 $\mathrm{Kg} / \mathrm{m} 2, \mathrm{SGA}$ class A and eGFR $>90 \mathrm{ml} / \mathrm{min} / 1.73 \mathrm{~m} 2$ by the CKD-EPI equation. Heart failure, chronic obstructive pulmonary disease, chronic liver disease, chronic use of corticosteroids, malignancy, proteinuria, or diabetes mellitus were considered as exclusion criteria.

C - One hundred twenty-one adult ESRD-HD patients with at least 6-months dialysis vintage. Malignancy or conditions with mandatory immobilization (e.g., amputation) were considered as exclusion criteria.

D - Thirty additional ESRD-HD patients were enrolled for the comparison between US muscle measurements taken at the start and immediately after the end of the dialysis session

\section{ULTRASONOGRAPHIC TECHNIQUE}

QRFT and QVIT were measured by experienced assessors (renal dietician or nephrologists) using B-mode, wall-tracking US systems (Philips HD7xe, Logiq and General Electric) and 7.5 $\mathrm{MHz}$ linear array transducers. The transducer was placed perpendicular to the long axis of the thigh with abundant use of contact gel and minimal pressure to avoid compression of the muscle. Measurements were performed in a standardized way at the level of two specific landmarks, the midpoint and the border between the lower third and the upper two-thirds between the superior anterior iliac spine and the upper pole of the patella, as previously described (9). The right and left quadriceps femoris muscle thickness were measured in both legs with the patient lying in a supine position, with both knees extended but relaxed and toes pointing upwards. Assessors performed two measurements at the level of each landmark (for a total of 8 measurements in each subject) immediately before each hemodialysis session, 
and the average of the values obtained for each site was used in the analyses. QRFT and QVIT were measured at the internal limit of the muscle. For the comparison between measurements performed before and after hemodialysis, measurements were performed by the same assessor immediately before and within 15 minutes after the end of each session.

\section{NUTRITIONAL STATUS EVALUATION}

Patients with ESRD were independently evaluated by a different assessor for protein-energy wasting (PEW) using the International-Society for Renal Nutrition and Metabolism (ISRNM) recommendations (10), and a PEW diagnosis was made accordingly.

In addition, the SGA status was defined (11) and the malnutrition inflammation score (MIS) was calculated (12).

\section{DEMOGRAPHIC AND CLINICAL VARIABLES}

Demographic, clinical and anthropometric data (height, body weight, BMI, triceps skinfold and mid-arm circumference) were collected for all groups. In ESRD patients, dialysis characteristics, chronic comorbidities and laboratory data were also collected.

\section{STATISTICAL ANALYSIS}

Based on the data from a previous study (9) we estimated that sample sizes of 43 and 129 in the control and ESRD group would achieve an approximate $80 \%$ power to detect a difference of $-0.2 \mathrm{~cm}$ between the null hypothesis that both groups means are $0.12 \mathrm{~cm}$ and the alternative hypothesis that the mean of the control group is $0.14 \mathrm{~cm}$ when the estimated common standard deviation is $0.4 \mathrm{~cm}$ (i.e. that the effect size of the difference between ESRD-HD and the control group is 0.50 ) and the significance level is 0.05 , using a two-sided two-sample t-test.

Data are expressed as mean and standard deviation, or median and range for continuous variables, as appropriate. Categorical variables are expressed as frequencies (percentage). To analyze differences in muscle thickness among groups of patients we fitted a linear mixed model with group as main factor (i.e. ESRD-HD patients, hospitalized patients and healthy subjects) and age, gender and $\mathrm{BMI}$ as covariates. In further analyses exploring differences in muscle thickness in ESRD-HD patients after stratification based on conventional diagnostic criteria for PEW, we fitted linear mixed models with subgroup as main factor and age, gender and BMI (stratification by serum albumin or MIS) or age and gender (stratification by BMI) as covariates.

The correlation between nutritional variables (BMI, Albumin, MIS score and arm muscle area) and ultrasound was assessed by Pearson's correlation coefficient for parametric data and Spearman correlation coefficient for non-parametric data. 
For the comparison between measurements performed before and after the hemodialysis session, we performed a paired t-test. Data analysis was performed using SPSS (Version 23, Armonk, NY: IBM Corp. USA).

\section{RESULTS}

\section{CLINICAL AND DEMOGRAPHIC VARIABLES}

Both ESRD-HD patients and hospitalized patients were significantly older compared to the healthy subjects control group. Male patients were more prevalent in the ESRD-HD group. BMI values were not significantly different among the three groups (Table 1).

I

Table 1 - Demographic and anthropometric data of healthy subjects, hospitalized patients and ESRDHD patients

\begin{tabular}{lllll} 
& $\begin{array}{l}\text { Healthy subjects } \\
(\mathbf{n = 3 5 )}\end{array}$ & $\begin{array}{l}\text { Hospitalized patients } \\
(\mathbf{n = 3 0 )}\end{array}$ & $\begin{array}{l}\text { ESRD-HD } \\
(\mathbf{n = 1 2 1})\end{array}$ & ANOVA \\
\hline Age (SD) & $41(10.0)^{\wedge}$ & $63(17.2)^{\star}$ & $67(16)$ & $<0.001$ \\
\hline Gender (male) \% & $15 / 35(43 \%)^{\#}$ & $16 / 30(53 \%)^{\star}$ & $79 / 121(65 \%)$ & 0.046 \\
\hline Height, cm (SD) & $170(9)^{\star}$ & $169(10)^{*}$ & $166(9)$ & 0.050 \\
\hline Weight, Kg (SD) & $70.5(16.6)$ & $75.4(16.2)^{\#}$ & $68.1(12.4)$ & 0.037 \\
\hline BMI (SD) & $24.3(4.6)^{\star}$ & $26.4(4.8)^{\star}$ & $24.9(4.6)$ & 0.16
\end{tabular}

Data are expressed as mean (standard deviation). Gender is reported as percentage of males. $\wedge \mathrm{P}<0.001$ vs ESRD, $\# \mathrm{P}<0.05$ vs ESRD, *non-statistically significant vs ESRD, post-hoc multiple comparisons with Dunn's test.

BMI, body mass index; ESRD, end-stage renal disease.

Clinical, metabolic and nutritional variables of the entire ESRD-HD cohort are reported in Table 2. On average, these patients had a fair dietary protein intake (DPI) $(1.11$ (SD 0.27) g/Kg/ day), low serum albumin (3.63 (SD 0.39) g/dl), and only a limited number of cases of PEW $(14 \%$ (17/121)) as diagnosed by the ISRNM criteria.

Table 2. Clinical, metabolic and nutritional variables of ESRD-HD patients.

\begin{tabular}{ll} 
Variable & All patients $(\mathbf{n}=\mathbf{1 2 1})$ \\
\hline Laboratory data & \\
\hline $\mathrm{BUN}(\mathrm{mg} / \mathrm{dl})$ & $48(20)$ \\
\hline $\mathrm{SCr}(\mathrm{mg} / \mathrm{dl})$ & $8.8(3.4)$ \\
\hline Transferrin $(\mathrm{mg} / \mathrm{dl})$ & $192(43)$ \\
\hline $\mathrm{CRP}(\mathrm{mg} / \mathrm{l})(\mathrm{median}($ range $))$ & $1.41(0.0-191.7)$ \\
\hline Albumin $(\mathrm{g} / \mathrm{dl})$ & $3.63(0.39)$ \\
\hline $\mathrm{DPI}(\mathrm{g} / \mathrm{Kg} / \mathrm{day})$ & $1.11(0.27)$ \\
\hline
\end{tabular}


Table 2. Continued

\begin{tabular}{ll} 
Variable & All patients $(\mathbf{n}=\mathbf{1 2 1})$ \\
\hline Cholesterol (mg/dl) & $155(41)$ \\
\hline Triglycerides (mg/dl) & $158(90)$ \\
\hline Diabetes mellitus (\%) & $30 / 121(25)$ \\
\hline COPD (\%) & $11 / 121(9)$ \\
\hline Peripheral vascular disease (\%) & $36 / 121(30)$ \\
\hline Cerebral vasculopathy (\%) & $25 / 121(21)$ \\
\hline Coronary artery disease (\%) & $30 / 121(25)$ \\
\hline Chronic heart failure (\%) & $11 / 121(9)$ \\
\hline Nutritional status & \\
\hline SGA score & $29(4.7)$ \\
\hline MIS score (median (range) & $6(1-22)$ \\
\hline AMA (cm $\left.{ }^{2}\right)$ & $47.9(13.1)$ \\
\hline Body weight (Kg) & $68.1(12.4)$ \\
\hline BMI & $24.9(4.6)$ \\
\hline PEW status (\%) & $17 / 121(14)$
\end{tabular}

Data are expressed as mean (standard deviation), except when differently specified.

AMA, Arm muscle area; BMI, Body mass index; BUN, Blood urea nitrogen; COPD, Chronic obstructive pulmonary disease; CRP, C-reactive protein; DPI, Dietary protein intake; MIS, Malnutrition inflammation score; PEW, Protein energy wasting; sCr, Serum creatinine; SGA, Subjective global assessment.

Table 3 illustrates the clinical and nutritional data of ESRD-HD patients when stratified by variables commonly used for the evaluation of the nutritional status in this clinical setting (BMI, albumin and MIS score status). No difference in DPI was found between subgroups (BMI $\geq 23 \mathrm{Kg} / \mathrm{m} 2$ vs $\mathrm{BMI}<23 \mathrm{Kg} / \mathrm{m2}: 1.08$ ( SD 0.06) vs 1.12 ( $\mathrm{SD} 0.29$ ), $\mathrm{P}=0.40$; albumin $\geq 3.8 \mathrm{~g} / \mathrm{dL}$ vs albumin < $3.8 \mathrm{~g} / \mathrm{dL}: 1.09$ (SD 0.07) vs 1.14 (SD 0.26), $\mathrm{P}=0.30$; MIS score $\geq 6$ vs MIS score < 6 : 1.08 (SD 0.25) vs 1.14 (SD 0.29), $P=0.25$ ). There was no significant difference in inflammatory status as assessed by CRP; on the other hand, predictably, all of the variables used for the assessment of nutritional status were different between the BMI, and MIS subgroups. No differences were found in BMI and arm muscle area when ESRD-HD patients were stratified according to albumin status. Conversely, when patients were stratified according to the MIS score, $B M I(P<0.001)$ and arm muscle area $(P=0.004)$ were significantly different between the subgroups; significant differences between the MIS subgroups were also found in some metabolic variables, namely blood urea nitrogen $(P<0.001)$, serum creatinine $(P=0.003)$, serum transferrin $(P<0.001)$, and albumin $(P<0.001)$. 


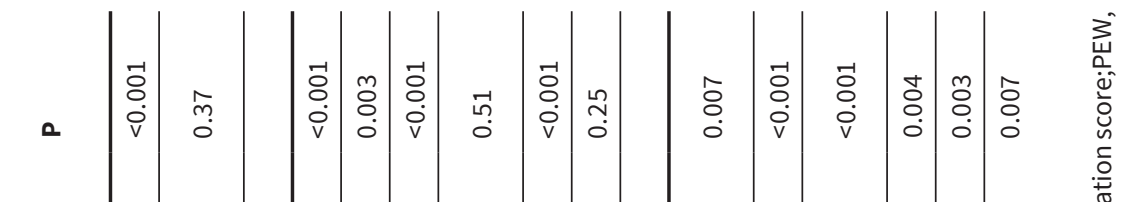

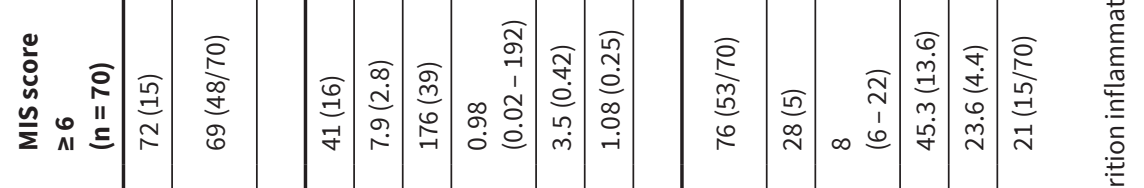

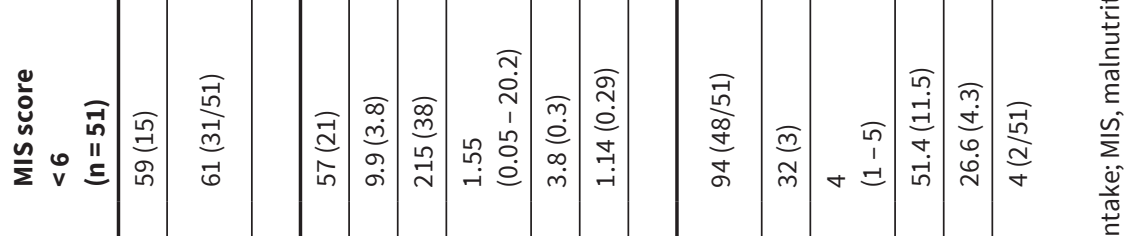

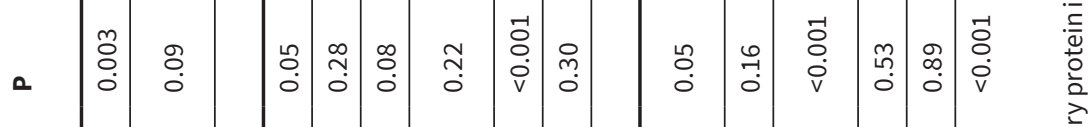

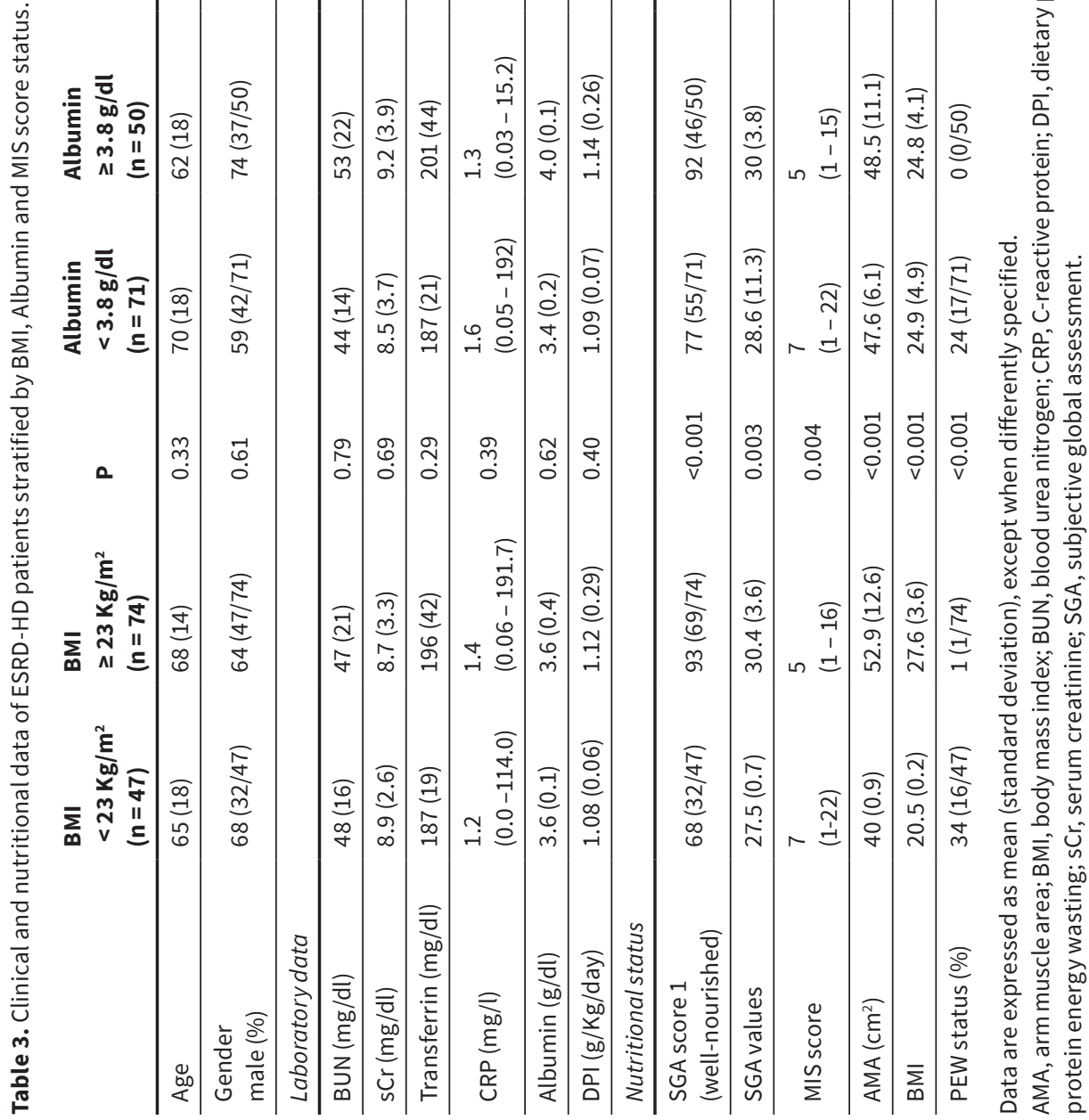




\section{COMPARISON BETWEEN US MEASUREMENTS PERFORMED BEFORE AND AFTER THE HEMODIALYSIS SESSION}

Figure 1 illustrates the mean muscle thickness for both measurements. No significant difference was found between measurements performed before and after the hemodialysis session. Weight change was $-2.25(-0.5--3.6) \mathrm{Kg}$. We divided patients into 2 groups based on intradialytic weight-loss to assess $(<-2.25 \mathrm{Kg}$ and $>-2.25 \mathrm{Kg}$ ) whether the amount of fluid removed could influence US measurements, no significant difference was found in neither the groups (data not shown). Demographic and clinical characteristics of the 30 patients enrolled in this part of the study are shown in the Supplementary Table 1.

Figure 1. Comparison between US measurements performed before and after the HD session.

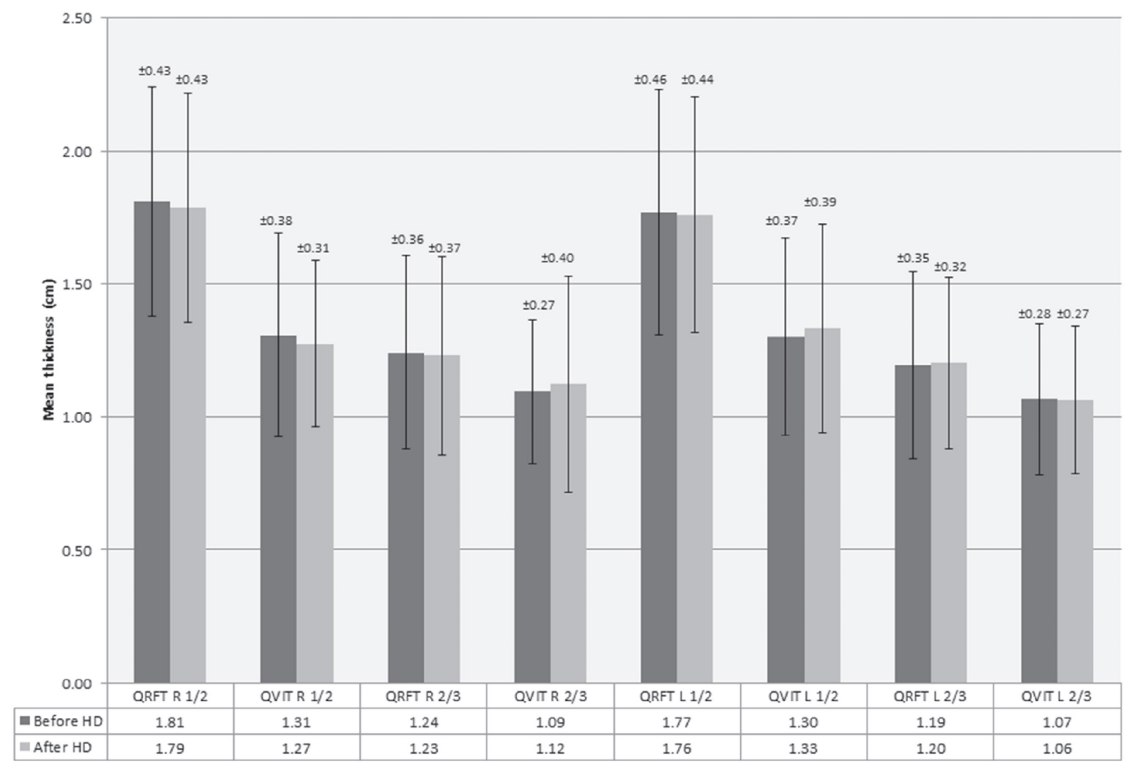

Legend. Data are expressed as mean and standard deviation. No significant difference was found between measurements. HD, hemodialysis; US, ultrasound.

\section{ULTRASONOGRAPHIC MEASUREMENTS OF MUSCLE THICKNESS}

Figure 2 illustrates the values of QRFT and QVIT in ESRD-HD patients, hospitalized patients and healthy subjects. All measures of muscle thickness were significantly lower at all sites in ESRD-HD patients compared with the two control groups. After adjusting for gender, age and BMI, QRFT and QVIT of ESRD-HD patients remained significantly lower at all sites as compared to the healthy subjects group. 
Figure 2. Ultrasound measurements of QRFT and QVIT of ESRD-HD, healthy subjects and hospitalized patients

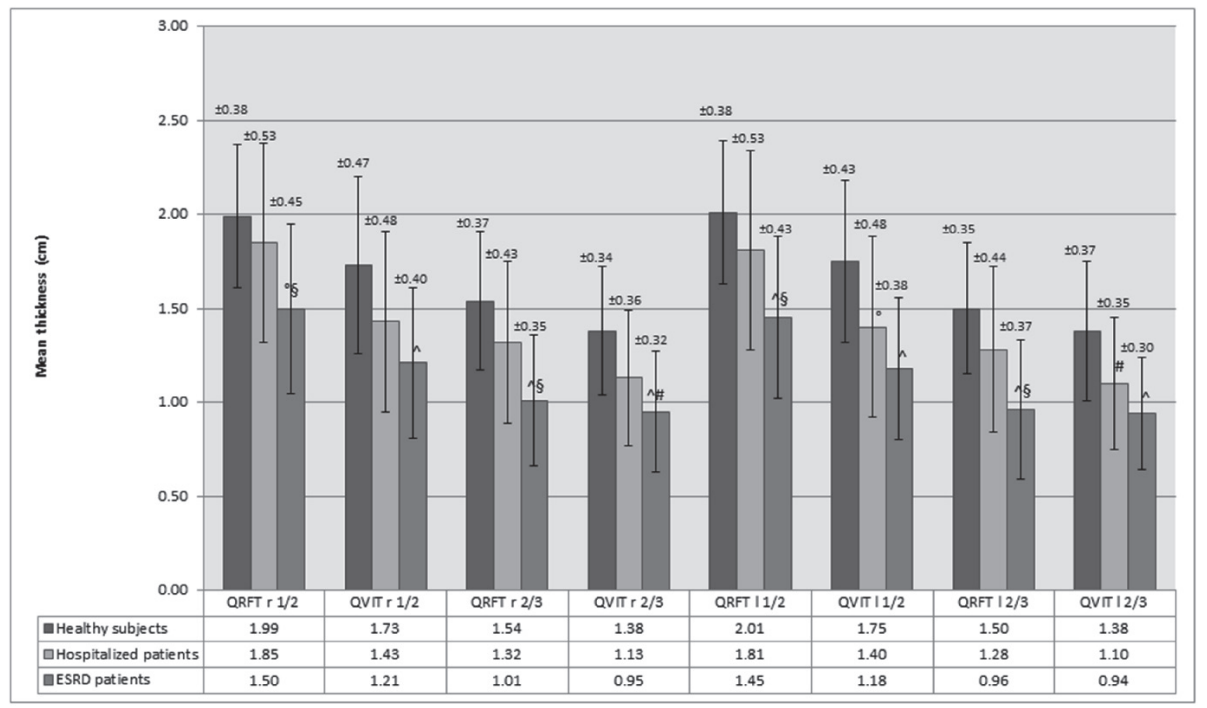

Legend. Data are expressed as mean (standard deviation). $\mathrm{P}<0.001$ adjusted for age, sex and BMI. ${ }^{\circ}$ $\mathrm{P}<0.01$, ^ $\mathrm{P}<0.001$ vs healthy subjects; \# $\mathrm{P}<0.05$, $\S \mathrm{P}<0.001$ vs hospitalized patients. Post-hoc pairwise comparisons by Sidak's test based on estimated marginal means in the mixed linear model. BMI, body mass index; ESRD-HD, End-Stage Renal Disease; QRFT, quadriceps rectus femoris thickness; QVIT; quadriceps vastus intermedius thickness.

Patients with a $\mathrm{BMI}<23 \mathrm{Kg} / \mathrm{m} 2$ showed decreased quadriceps muscle thickness at all of the explored sites compared to well-nourished patients. The differences in QVIT were greater than the differences in the QRFT, but all differences achieved statistical significance (Figure 2A). At multivariable analysis, the adjusted differences in muscle thickness between the MIS subgroups were highly statistically significant (Figure 2C). Conversely, no differences were found in muscle thickness between patients with serum albumin $<3.8 \mathrm{~g} / \mathrm{dL}$ compared to those with serum albumin $>3.8 \mathrm{~g} / \mathrm{dL}$ (Figure $2 \mathrm{~B}$ ). 
Figure 3 (A-C). QRFT and QVIT of ESRD-HD patients stratified based on BMI, albumin and MIS score status.

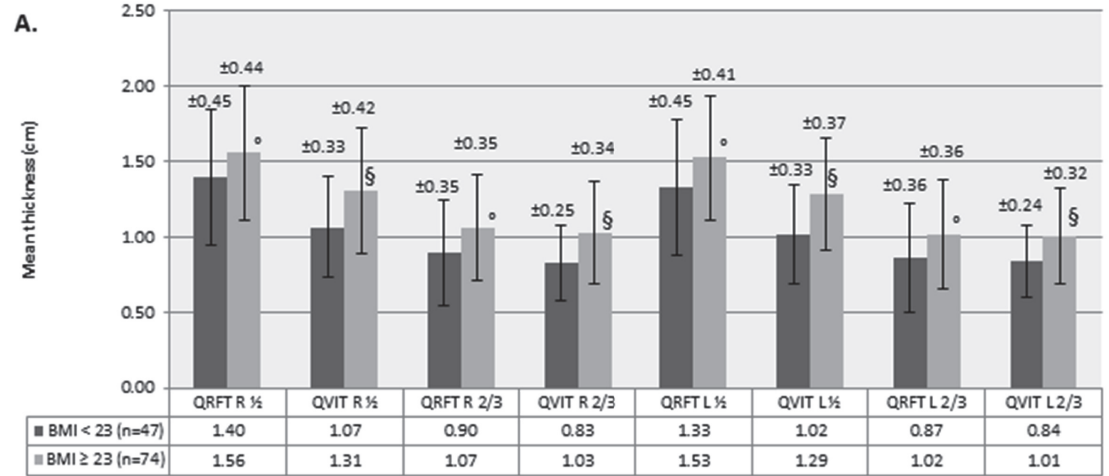

B.

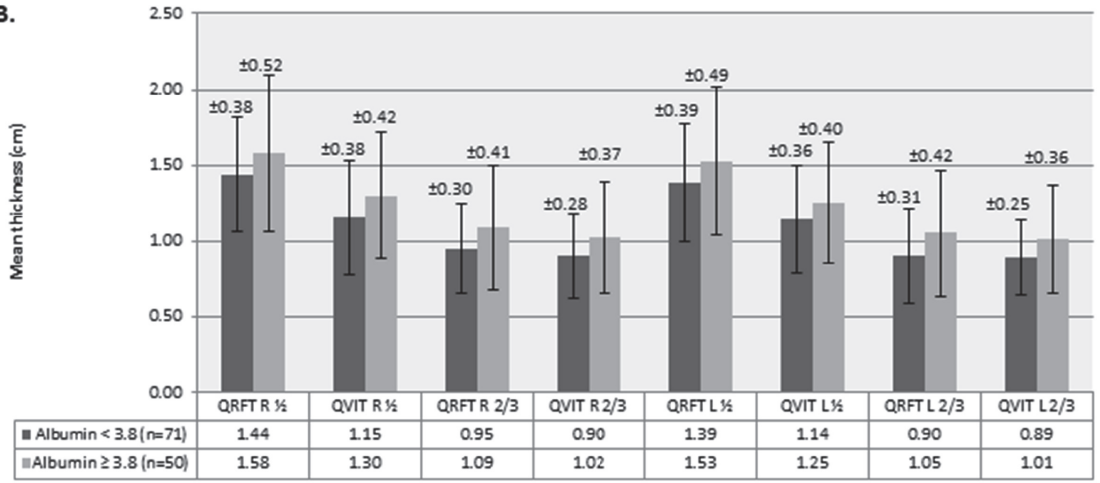

c.

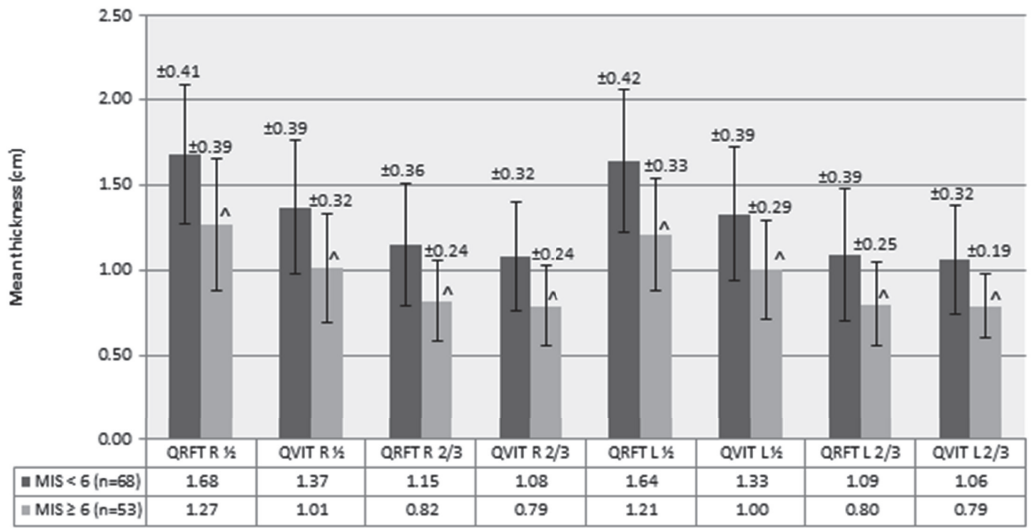

Legends. Data are expressed as mean and standard deviation. BMI, Body mass index; ESRD, End-stage renal disease; MIS, Malnutrition inflammation score; QRFT, Quadriceps rectus femoris thickness; QVIT, Quadriceps vastus intermedius thickness. Figure 3 A. ESRD patients stratified by BMI. $P<0.01$, adjusted for age and gender. ${ }^{\circ} \mathrm{P}<0.01, \S \mathrm{P}<0.001$ vs $\mathrm{BMI}<23$. Post-hoc pairwise comparisons by Sidak's test based on estimated marginal means in the mixed linear models. Figure 3B. ESRD patients stratified by albumin. No significant difference between groups, analysis adjusted for age, gender and BMI.

Figure 3C. ESRD patients stratified by MIS score. ${ }^{\wedge} \mathrm{P}<0.001$ vs MIS $<6$. Post-hoc pairwise comparisons by Sidak's test based on estimated marginal means in the mixed linear models. 


\section{CORRELATION BETWEEN CONVENTIONAL NUTRITIONAL VARIABLES AND QUADRICEPS MUSCLE THICKNESS ASSESSED BY US}

Muscle ultrasonographic indexes were significantly and negatively correlated with the MIS score ( $r=-0.37$ to $-0.47, P<0.001$ for all sites of measurement). $B M I$ ( $r=0.19$ to $0.36, P=0.04$ to $P<0.001$ for all sites of measurement), albumin ( $r=0.19$ to $0.27, P=0.03$ to $P=0.003$ for all sites of measurement), and arm muscle area ( $r=0.24$ to $0.36, P=0.005$ to $P<0.001$ for all sites of measurement) had a weaker though significant positive correlation to quadriceps muscle thickness as assessed by US.

\section{DISCUSSION}

The main finding of this study is that ESRD-HD had lower quadriceps muscle thickness, as assessed by US measurement, in comparison to both younger healthy subjects and well-nourished hospitalized patients with normal renal function without acute or chronic comorbidities known to negatively influence nutritional status. This finding is unchanged even after adjusting for sex, age and BMI.

Our findings confirm recent data obtained in patients with chronic kidney disease (CKD) on conservative treatment, indicating that the prevalence of sarcopenia as assessed by DEXA was higher among patients with lower eGFR values and $\geq 60$ years old (14). Moreover, our findings are in agreement with the results of another study performed with MRI, reporting that the volume of quadriceps muscle was significantly lower in ESRD-HD compared to controls (15).

When ESRD-HD patients are stratified for different indexes of nutritional status, muscle mass, as estimated by US measurement of quadriceps muscle thickness, was significantly decreased in malnourished patients. Adopting the ISRNM cut-off values for BMI clearly identified patients with reduced quadriceps muscle thickness and other altered nutritional variables, such as arm muscle area, SGA and MIS score. Similarly to the BMI, patients in the subgroup of the worse MIS score ( $\geq 6$ ) had lower quadriceps muscle thickness, arm muscle area, BMI and SGA score, but also lower albumin and total transferrin levels. However, since albumin, transferrin, BMI and the SGA are part of the MIS, these results were not unexpected. On the other hand, stratification of ESRD-HD patients based on albumin cut-off values did not reveal significant differences in quadriceps muscle thickness, BMI or arm muscle area reinforcing the poor value of albumin for nutritional status evaluation in ESRD patients on hemodialysis, since this variable is known to be also influenced by the inflammatory status typical of ESRD-HD patients $(16,17)$.

We found low to moderate statistically significant correlations between muscle thickness measured by US and other nutritional variables (MIS, arm muscle area, albumin and BMI), with the strongest correlation being found between US measurements and the MIS score. A partial explanation for this finding may reside in the fact that the MIS score is a composite of different nutritional variables, while other variables assessed in this study do not directly reflect (e.g., 
albumin and BMI), or measure (e.g., arm muscle area) muscle mass. Although BMI was found to be significantly correlated $(r=0.69, P<0.0001)$ with total body muscularity, as assessed by US measurements of muscle thickness at 9 body sites (18), a lower correlation with the muscle mass of one single limb may be expected.

Similarly to our study on patients with AKI (9), we confirmed that US measurements of the quadriceps femoris muscle are not influenced by rapid fluid shifts, even in chronically hyperhydrated patients.

We are not aware of other studies investigating US measurements of quadriceps muscle thickness in ESRD-HD patients on hemodialysis. Recently, we reported the high reliability and ease of this approach in a different group of patients with kidney disease, i.e., critically ill patients with Acute Kidney Injury (9).

It is important to address the limitations of our study. Firstly, no validation of US against a gold standard technique such as CT or MRI was obtained in our ESRD-HD patients. However, the US method for the evaluation of quadriceps femoris muscle has been validated in other clinical settings, such as COPD and coronary artery disease patients $(7,19)$. Secondly, since this was planned as an observational cross-sectional study, we could not explore the prognostic value of quadriceps muscle thickness in ESRD-HD. Nevertheless, a recent study in a different clinical setting, suggests that reduction in the rectus femoris area measured by US could represent an independent risk factor for hospital readmission and longer hospitalization (20). Thirdly, in addition to PEW, other factors are known negatively affect muscle mass, with the most relevant being the level of physical activity (21), a variable not taken into account in our study. Literature data indicate that in hemodialysis patients a sedentary lifestyle is predominant (22-25), and this could represent an important factor contributing to the reduced muscularity found in our study. Nevertheless, the stratification of our ESRD-HD patients into groups based on other nutritional parameters confirmed the lower muscularity in those with worse nutritional status. In addition, the prevalent sedentary lifestyle, and consequently lower muscularity, adds to the increased risk for sarcopenia of ESRD patients. Lastly, we choose two different control groups, young healthy subjects and well-nourished hospitalized patients with normal renal function. Despite the fact that our groups were not matched by age and sex, statistical analysis performed by adjusting for those two factors plus BMI, confirmed the difference between ESRD patients and healthy subjects.

The potential for US evaluation of skeletal muscles in ESRD-HD makes it possible to foresee its application to other important research topics. In a recent study in patients with Chronic Kidney Disease on conservative treatment (i.e. not yet started on dialysis), an increase in the area of the rectus femoris muscle measured by US was demonstrated in patients undergoing a physical exercise program compared to sedentary patients matched by age and comorbidities (26). Together with the early identification of patients in risk for PEW, the ability to directly measure the effectiveness of nutritional interventions and of physical activity intervention 
programs aimed at improving muscle trophism and mass, could strongly support a more extensive application of muscle US in renal patients.

In conclusion, quadriceps muscle US is a simple technique, easily applicable at the bedside in dialysis units, and suitable for the identification of ESRD-HD patients with reduced muscle mass. Since US is widely available in the Nephrology setting, has a low cost, does not require dedicated staff (radiologists), is non-invasive, and does not provide radiation exposure, it could become a particularly useful tool for bedside evaluation of muscle mass. This could allow an early identification of patients at risk for PEW, as well as a close monitoring of nutritional and rehabilitative interventions aimed at increasing lean body mass.

\section{STATEMENT OF AUTHORSHIP}

All authors certify that they have participated sufficiently in the work to take public responsibility for the content, including participation in the concept, design, analysis, writing, or revision of the manuscript.

\section{CONFLICT OF INTEREST STATEMENT}

Authors declare no conflict of interest.

\section{FUNDING SOURCES}

Alice Sabatino is the recipient of a young investigator research grant by the Italian Society of Parenteral and Enteral Nutrition (SINPE, Società Italiana di Nutrizione Parenterale ed Enterale) for the project: "Valutazione nutrizionale nell'insufficienza renale mediante ecografia del muscolo quadricipite femorale" "Nutritional assessment of patients with chronic kidney disease and acute kidney injury through ultrasound of the quadriceps femoris muscle"). 


\section{REFERENCES OF CHAPTER 4}

1. Ikizler TA, Cano NJ, Franch H, et al. Prevention and treatment of protein energy wasting in chronic kidney disease patients: a consensus statement by the International Society of Renal Nutrition and Metabolism. Kidney Int 2013;84(6):1096-1107

2. Sabatino A, Regolisti G, Karupaiah T, et al. Protein-energy wasting and nutritional supplementation in patients with end-stage renal disease on hemodialysis. Clin Nutr 2017;36(3):663-671

3. Carrero JJ, Johansen KL, Lindholm B, et al. Screening for muscle wasting and dysfunction in patients with chronic kidney disease. Kidney Int 2016;90(1):53-66

4. Mourtzakis M, Wischmeyer P. Bedside ultrasound measurement of skeletal muscle. Curr Opin Clin Nutr Metab Care 2014;17(5):389-395

5. Smith S, Madden AM. Body composition and functional assessment of nutritional status in adults: a narrative review of imaging, impedance, strength and functional techniques. J Hum Nutr Diet 2016;29(6):714-732

6. Menon MK, Houchen L, Harrison S, et al. Ultrasound assessment of lower limb muscle mass in response to resistance training in COPD. Respir Res 2012;13:119

7. Seymour JM, Ward K, Sidhu PS, et al. Ultrasound measurement of rectus femoris cross-sectional area and the relationship with quadriceps strength in COPD. Thorax 2009;64(5):418-423

8. Arbeille P, Kerbeci P, Capri A, et al. Quantification of muscle volume by echography: comparison with MRI data on subjects in long-term bed rest. Ultrasound Med Biol 2009;35(7):1092-1097

9. Sabatino A, Regolisti G, Bozzoli L, et al. Reliability of bedside ultrasound for measurement of quadriceps muscle thickness in critically ill patients with acute kidney injury. Clin Nutr 2017, in press

10. Fouque D, Kalantar-Zadeh K, Kopple J, et al. A proposed nomenclature and diagnostic criteria for protein-energy wasting in acute and chronic kidney disease. Kidney Int 2008;73(4):391-398

11. Visser R, Dekker FW, Boeschoten EW, et al. Reliability of the 7-point subjective global assessment scale in assessing nutritional status of dialysis patients. Adv Perit Dial 1999;15:222-225

12. Kalantar-Zadeh $\mathrm{K}$, Kleiner M, Dunne E, et al. A modified quantitative subjective global assessment of nutrition for dialysis patients. Nephrol Dial Transplant 1999;14(7):1732-1738

13. Baumgartner RN, Waters DL, Gallagher D, et al. Predictors of skeletal muscle mass in elderly men and women. Mech Ageing Dev 1999;107(2):123-136

14. Sharma D, Hawkins M, Abramowitz MK. Association of sarcopenia with eGFR and misclassification of obesity in adults with CKD in the United States. Clin J Am Soc Nephrol 2014;9(12):2079-2088

15. Segura-Ortí E, Gordon PL, Doyle JW, et al. Correlates of Physical Functioning and Performance Across the Spectrum of Kidney Function. Clin Nurs Res 2017:1054773816689282 
16. Gama-Axelsson T, Heimbürger $O$, Stenvinkel $P$, et al. Serum albumin as predictor of nutritional status in patients with ESRD. Clin J Am Soc Nephrol 2012;7(9):1446-1453

17. Kaysen GA, Dubin JA, Müller HG, et al. Inflammation and reduced albumin synthesis associated with stable decline in serum albumin in hemodialysis patients. Kidney Int 2004;65(4):1408-1415

18. Kanehisa H, Fukunaga T. Association between body mass index and muscularity in healthy older Japanese women and men. J Physiol Anthropol 2013;32(1):4

19. Thomaes T, Thomis M, Onkelinx S, et al. Reliability and validity of the ultrasound technique to measure the rectus femoris muscle diameter in older CAD-patients. BMC Med Imaging 2012;12:7

20. Greening NJ, Harvey-Dunstan TC, Chaplin EJ, et al. Bedside assessment of quadriceps muscle by ultrasound after admission for acute exacerbations of chronic respiratory disease. Am J Respir Crit Care Med 2015;192(7):810-816

21. Burd NA, Gorissen SH, van Loon LJ. Anabolic resistance of muscle protein synthesis with aging. Exerc Sport Sci Rev 2013;41(3):169-173

22. Painter P, Marcus RL. Assessing physical function and physical activity in patients with CKD. Clin J Am Soc Nephrol 2013;8(5):861-872

23. Fiaccadori E, Sabatino A, Schito F, et al. Barriers to physical activity in chronic hemodialysis patients: a single-center pilot study in an Italian dialysis facility. Kidney Blood Press Res 2014;39(2-3):169-175

24. Broers NJH, Martens RJH, Cornelis T, et al. Physical Activity in End-Stage Renal Disease Patients: The Effects of Starting Dialysis in the First 6 Months after the Transition Period. Nephron 2017

25. Shimoda T, Matsuzawa R, Yoneki K, et al. Changes in physical activity and risk of all-cause mortality in patients on maintence hemodialysis: a retrospective cohort study. BMC Nephrol 2017;18(1):154

26. Watson EL, Greening NJ, Viana JL, et al. Progressive Resistance Exercise Training in CKD: A Feasibility Study. Am J Kidney Dis 2015;66(2):249-257 
Muscle ultrasound in ESKD 73 



\section{CHAPTER 5}

\section{Ultrasound for non-invasive assessment and monitoring of quadriceps muscle thickness in critically ill patients with Acute Kidney Injury}




\section{ABSTRACT}

Background and aims: Critically ill patients with acute kidney injury (AKI) undergo major muscle wasting in the first few days of ICU stay. An important concern in this clinical setting is the lack of adequate tools for routine bedside evaluation of the skeletal muscle mass, both for the determination of nutritional status at admission, and for monitoring. In this regard, the present study aims to ascertain if ultrasound (US) is able to detect changes in quadriceps muscle thickness of critically ill patients with acute kidney injury (AKI) over short periods of time.

Methods: This is a prospective observational study with a follow-up at 5 days. All adult patients with AKI hospitalized at the Renal ICU of the Parma University Hospital over 12 months, with a hospital stay before ICU admission no longer than $72 \mathrm{~h}$, and with a planned ICU stay of at least 5 days, were eligible for the study. An experienced investigator assessed quadriceps rectus femoris and vastus intermedius thickness (QRFT and QVIT) at baseline and after 5 days of ICU stay.

Results: We enrolled 30 patients with $74 \pm 11$ years of age and APACHE II score of $22 \pm 5$. Muscle thickness decreased by $15 \% \pm 13 \%$ within the first 5 days of ICU stay $(\mathrm{P}<0.001$ for all sites as compared to ICU admission). Patients with more severe muscle loss had a higher probability of prolonged hospitalization.

Conclusions: In critically ill patients with AKI, bedside muscle US identifies patients with accelerated muscle wasting.

Key words: Acute Kidney Injury; body composition; critical care; intensive care unit; muscle wasting; muscle ultrasound 


\section{INTRODUCTION}

Critically ill patients undergo major muscle wasting in the first few days of their ICU stay (1). Clinical consequences are represented by delayed functional recovery, difficult weaning from mechanical ventilation and increased mortality risk (1).

In this clinical setting an important cause of concern is the lack of adequate tools for routine bedside evaluation of the skeletal muscle mass (2). The reference methods considered as the gold standard for the assessment of skeletal muscle, such as computed tomography (CT), magnetic resonance imaging (MRI) and dual energy X-ray absorptiometry (DEXA) are not feasible for routine evaluation and monitoring of muscle mass and body composition (2). On the other hand, currently used bedside tools, such as bioimpedance analysis (BIA) and anthropometry, are not accurate enough in critically ill patients (2), mainly due to the possible interference of fluid overload, frequently observed especially when Acute Kidney Injury (AKI) coexists. Recently, the use of ultrasound (US) for the assessment of muscle dimensions has aroused considerable interest, and its reliability and validity have been documented also in critically ill patients with AKI $(3,4)$. US technique seems to be poorly influenced not only by fluid overload, but also by the rapid and relevant fluid shifts typical of patients with AKI undergoing Kidney Replacement Therapy (KRT) (3).

On this premise, in the present study we aimed to assess whether US is able to detect changes in muscle thickness of patients with AKI over a short period of time.

\section{MATERIALS AND METHODS}

\section{PATIENTS}

This is a prospective, longitudinal (5 days) observational study, conducted in the Renal ICU of the Parma University Hospital. Procedures were held in accordance to the Helsinki declaration and informed consent was obtained from patients or their next of kin. The study was approved by the local ethics committee (Comitato Etico di Area Vasta Emilia Nord, AVEN, Prot n. 43943 -03/12/2015).

All adult patients with AKI hospitalized in the Renal ICU from 15/03/2017 to 15/03/2018, with a hospital stay before ICU admission no longer than 72 hours, and with a predictable ICU stay of at least 5 days were eligible for the study. AKI was diagnosed according to KDIGO guideline criteria (5).

Already available data on quadriceps femoris US evaluation in healthy subjects (body mass index (BMI) $>18.5 \mathrm{Kg} / \mathrm{m} 2$, Subjective Global Assessment (SGA) class A, absence of chronic or acute illness) (6) were used for comparison with AKI patients, both at ICU admission and after 5 days of ICU stay. 


\section{METHODS}

US technique

The same experienced investigator (renal dietitian) performed all of the measurements. Quadriceps rectus femoris thickness (QRFT) and quadriceps vastus intermedius thickness (QVIT) were measured by B-mode ultrasonography, wall tracking ultrasound system (Philips hd7xe) with a 7.5 MHz linear array transducer (L12-3 transducer), as previously described in detail (3). All US measurements were performed in duplicate and the average of the scores used in final analyses. The transducer was placed perpendicular to the long axis of the thigh with a large amount of gel and no pressure to avoid compression of the muscle. QRFT and QVIT were measured at the midpoint (RF Prox; VI Prox) and at the border between the upper third and lower two-thirds (RF Dist; Prox Dist) between the anterior superior iliac spine (ASIS) and the upper pole of the patella $(3,7)$. The right and left quadriceps values were assessed in both legs with the patient lying in a supine position with both knees extended but relaxed and toes pointing to the ceiling. The assessor was positioned on the side of the patient while performing the measurements, and was allowed to tilt the probe to obtain the best possible image, in which RF and VI would be aligned and centered. Measurements were performed directly on the US machine while obtaining the images. The vertical diameter of the muscles was measured on the inner edge of the muscle fascia. US was performed twice during ICU stay, at baseline (at ICU admission) and after 5 days since the first measurement. Muscle US took less than 20 minutes to perform the measurements and complete the image acquisition.

Demographics, clinical data, renal function and outcome: data were collected as per institutional routine at the time of ICU admission and during ICU stay, with special regard to demographic, body weight and height, clinical and laboratory data, renal function, acute and chronic comorbidities, severity of illness (APACHE II score), data on renal replacement therapy (RRT), length of stay and mortality.

- $\quad$ Outcomes: muscle loss after 5 days.

\section{STATISTICAL ANALYSIS}

Results are expressed as mean and standard deviation for continuous variables with normal distribution, or median and range for non-parametric data, and as frequencies for categorical variables. Group differences were analyzed using Student $t$ test and Mann-Whitney's U test for parametric and non-parametric data, respectively to assess difference between means of the control group and the patient group. ANCOVA was used to adjust the analysis by age and sex.

We examined the difference between muscle thickness at baseline and at 5 days after admission by mixed-effects models with patients fitted as random effects, and the four-way interaction term between time and each of the three sites of measurements (RF vs VI, Left vs Right, Proximal vs Distal) fitted as fixed effects. We examined the relation between baseline 
comorbidities and change in muscle thickness by mixed-effects ANCOVA models in which muscle thickness at 5 days after admission was included as dependent variate and baseline thickness was included as covariate, in order to adjust for the correlation between change in muscle thickness and random differences in baseline values. We examined the relation between change in muscle thickness and ICU outcome (discharge, transferal to other health care facility, death) in two steps. First, we estimated the individual change over time in muscle thickness by the best linear unbiased predictions (BLUPS) of the random slope from mixedeffects random coefficients models. Then, we fitted a multinomial logistic regression model where outcome (discharge, transferred to rehabilitation unit, death) was the dependent variable and the individual random slope the independent variable. Because of sparse data concerning mortality (five patients only) we did not report the findings on mortality. A twosided $P$ value of less than 0.05 was regarded as statistically significant. Stata Release 16 (StataCorp, College Station, TX, US) was used for all the analyses.

\section{SAMPLE SIZE CALCULATIONS}

No data are currently available in the literature on US evaluation and monitoring of quadriceps muscle mass in patients with AKI. In a recent study on ICU patients (8) 22 patients were enrolled in order to detect a $16 \%$ reduction in the quadriceps rectus femoris thickness after 5 days of ICU stay, with a power of $80 \%$ and a probability of type I error equal to 0.05 . We enrolled 30 patients to account for possible drop-outs.

\section{RESULTS}

Table 1 shows the baseline characteristics of the 30 patients studied. Seventy percent (21/30) were male with a mean \pm SD age of $74 \pm 11$ years, and they represented a severely critically ill cohort (APACHE II was $22 \pm 5$. A total of 472 images were analyzed across the 30 patients. Eighty-three percent of patients (25/30) were non-surgical patients and the main admission diagnosis in the ICU was renal, followed by sepsis. On average, patients were polymorbid $(2.8$ \pm 1.7 comorbidities per patient), hypertension being the most frequent comorbidity. As to the usual renal function, $37 \%(11 / 30$ ) had basal eGFR values $<60 \mathrm{ml} / \mathrm{min} / 1.73 \mathrm{~m} 2$ (CKD stages 2 to 5 non dialysis). At the time of first US evaluation, all of the patients had stage $3 \mathrm{AKI}$; in 21/30 patients (70\%) RRT was started as 10-12 hour lasting sustained low-efficiency dialysis. Oliguria was common (67\%), as was sepsis (40\%). ICU mortality was $17 \%(5 / 30)$; hospital mortality was $30 \%$ (9/30). The median (range) length of ICU stay was 15 (4-72) days, while the length of hospital stay was 34 (7-138). C-reactive protein was $109.2 \mathrm{mg} / \mathrm{dL}( \pm 68.1)$.

\section{PATIENTS WITH AKI IN COMPARISON TO HEALTHY SUBJECTS}

Demographic characteristics of control group (35 healthy subiects) are shown in Table 1, while US quadriceps muscle data are illustrated in Figure 1. In general, the control group was younger 
and leaner than patients. At univariate analysis (Figure 1), muscle thickness of patients differed from that of the control group for all sites, both at T1 and T2. We also performed an adjusted analysis using ANCOVA corrected for age and sex. In the adjusted analysis, no difference was found between T1 values of muscle thickness and control group values; however, the difference between $\mathrm{T} 2$ values and the control group values remained statistically significant.

Table 1. Demographic and clinical data.

\begin{tabular}{|c|c|c|}
\hline Variables & Patients $(n=30)$ & Healthy subjects $(n=35)$ \\
\hline Age & $74(10.6)$ & $41(10.0)^{\star}$ \\
\hline Male sex (n, \%) & $21 / 30(70)$ & $15 / 35(43)^{\star}$ \\
\hline Body weight (Kg) & $82(13.2)$ & $70.5(16.6)^{\star}$ \\
\hline Height (m) & $1.67(0.09)$ & $1.70(0.09)$ \\
\hline $\mathrm{BMI}\left(\mathrm{Kg} / \mathrm{m}^{2}\right)$ & $29(4.6)$ & $24.3(4.6)^{\star}$ \\
\hline APACHE II & $22(5)$ & NA \\
\hline \multicolumn{3}{|l|}{ Main admission diagnosis (n, \%) } \\
\hline - Renal & $18 / 30(60)$ & NA \\
\hline - Sepsis & $4 / 30(14)$ & NA \\
\hline - Respiratory & $3 / 30(10)$ & NA \\
\hline - Vascular & $3 / 30(10)$ & NA \\
\hline - Malignancy & $1 / 30(3)$ & NA \\
\hline - Cardiac & $1 / 30(3)$ & NA \\
\hline \multicolumn{3}{|l|}{ Surgical status (n, \%) } \\
\hline - Urgent & $2 / 30(7)$ & NA \\
\hline - Programmed & $3 / 30(10)$ & NA \\
\hline - Non-surgical & $25 / 30(83)$ & NA \\
\hline \multicolumn{3}{|l|}{ Chronic comorbidities (n, \%) } \\
\hline - Hypertension & $23 / 30(77)$ & NA \\
\hline - Diabetes mellitus & $11 / 30(37)$ & NA \\
\hline- COPD & $7 / 30(23)$ & NA \\
\hline - Ischemic cardiopathy & $7 / 30(23)$ & NA \\
\hline - Heart failure & $8 / 30(27)$ & NA \\
\hline - Peripheral vascular disease & $5 / 30(17)$ & NA \\
\hline - Immunocompromised & $2 / 30(7)$ & NA \\
\hline - Chronic liver disease & $2 / 30(7)$ & NA \\
\hline - Malignancy & $6 / 30(20)$ & NA \\
\hline - Chronic kidney disease (not on dialysis) & $11 / 30(37)$ & NA \\
\hline Acute complications at first muscle US (n, \%) & & NA \\
\hline - Sepsis & $12 / 30(40)$ & NA \\
\hline - Invasive mechanical ventilation & $4 / 30(13)$ & NA \\
\hline - Non-invasive mechanical ventilation & $7 / 30(23)$ & NA \\
\hline - Oliguria & $20 / 30(67)$ & NA \\
\hline - Vasoactive drug need & $7 / 30(23)$ & NA \\
\hline - Renal replacement therapy & $21 / 30(70)$ & NA \\
\hline \multicolumn{3}{|l|}{ ICU outcome (n, \%) } \\
\hline - Death & $5 / 30(17)$ & NA \\
\hline
\end{tabular}


Table 1. Continued

\begin{tabular}{lll} 
Variables & Patients $(\mathbf{n}=\mathbf{3 0})$ & Healthy subjects $(\mathbf{n}=\mathbf{3 5})$ \\
\hline Hospital outcome $(\mathrm{n}, \%)$ & & NA \\
$\quad$ - Death & $9 / 30(30)$ & NA \\
$\quad$ - Discharged home & $15 / 30(50)$ & NA \\
- Transferred to long-stay/rehabilitation ward & $2 / 30(7)$ & NA \\
$\quad$ - Transferred to another hospital & $4 / 30(13)$ & NA \\
\hline ICU LOS median, range) & $15(4-72)$ & NA \\
Hospital LOS (median, range) & $34(7-138)$ & \\
\hline Biochemical data & & NA \\
\hline SCr (mg/dl) & $6.3(4)$ & NA \\
\hline BUN (mg/dl) & $81.7(36)$ & NA \\
\hline Albumin $(g / d l)$ & $2.8(0.6)$ & NA
\end{tabular}

Data expressed as mean (standard deviation), frequencies and median (range). * $\mathrm{P}<0.001$ in comparison to AKI patients. BMI, body mass index; BUN, blood urea nitrogen; COPD, chronic obstructive pulmonary disease; CRP, C-reactive protein; ICU, intensive care unit; IMV, invasive mechanical ventilation; LOS, length of stay; NIMV, non-invasive mechanical ventilation; sCr, serum creatinine.

\section{CHANGES IN MUSCLE THICKNESS BY US}

Baseline (T1) and after 5 days (T2) mean \pm SD values for RF and VI thickness are illustrated in figure 1 ; the difference between means was statistically significant for all sites $(P<0.001)$. On average, there was a mean clinically relevant reduction $15 \%( \pm 12 \%)$ in every site of measurement within the first 5 days of ICU stay (Table 2). Changes in VI Prox and Dist thickness (16-19\%) were greater than in RF Prox and Dist thickness (11-13\%). 
Figure 1. Muscle thickness of patients with AKI (baseline, T1, and after 5 days, T2), and control group.

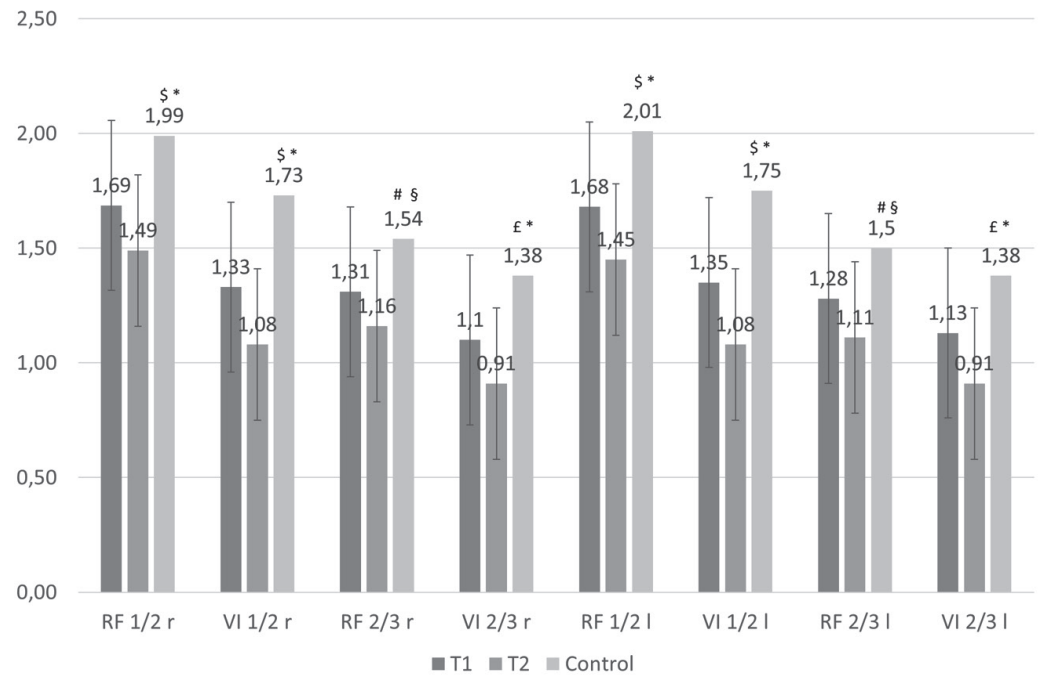

Legend. The first 2 columns represent the mean and 95 percent confidence interval (vertical bar) of muscle thickness at each measurement site as estimated by the mixed effect model, at baseline (T1) and after 5 days (T2). The third column represents the control group. The average difference between baseline and 5 days was statistically significant at all measurement sites $(P<0.001)$. When comparing the difference between patients and healthy subjects, muscle thickness was different between controls and baseline (T1) values of patients: $\$ P=0.001$, $\# P<0.05, P<0.01 ; P<0.001$ in comparison to muscle thickness of patients after 5 days (T2). After adjusting the analysis for age and sex using ANCOVA, no statistically significant difference was found between T1 values and controls; when comparing to T2 values, muscle thickness difference remained statistically significant for all sites: ${ }^{\star} P<0.01, \S P<0.05$.

Table 2. Average percent reduction of muscle thickness after 5 days of ICU stay

Muscle site

\begin{tabular}{ll}
\hline QRFT Prox $(n=29)$ & $11 \%(8 \%)$ \\
\hline QVIT Prox r $(n=28)$ & $18 \%(14 \%)$ \\
\hline QRFT Dist $r(n=30)$ & $11 \%(10 \%)$ \\
\hline QVIT Dist $r(n=30)$ & $17 \%(12 \%)$ \\
\hline QRFT Prox I $(n=30)$ & $13 \%(10 \%)$ \\
\hline QVIT Prox I $(n=29)$ & $19 \%(16 \%)$ \\
\hline QRFT Dist I $(n=30)$ & $12 \%(10 \%)$ \\
\hline QVIT Dist I $(n=30)$ & $16 \%(15 \%)$ \\
\hline All measurements $(n=236)$ & $15 \%(12 \%)$
\end{tabular}

Dist, distal; Prox, proximal; QRFT, quadriceps rectus femoris thickness; QVIT, quadriceps vastus intermedius thickness; Values expressed as mean (standard deviation). 
We did not find any association between baseline muscle thickness, inflammatory status (assessed by CRP and serum albumin), as well as chronic and acute comorbidities, with muscle loss (Supplementary table 1). Patients with the more severe muscle loss had a higher probability of having a prolonged hospital stay, which was determined by being transferred to rehabilitation unit instead of discharged home (OR: 0.04, 95\% Cl: 0.00- 0.74; $\mathrm{P}=0.031$ ) (Figure 2).

Supplementary table 1. Relation between baseline chronic and acute comorbidities and change in muscle thickness (ANCOVA).

\begin{tabular}{|c|c|c|}
\hline & Coefficient (SE) & 95\% Confidence interval, $\mathbf{P}$ \\
\hline \multicolumn{3}{|l|}{ Chronic comorbidities } \\
\hline Hypertension & $0.132(0.13)$ & $-0.25 ; 0.37,0.692$ \\
\hline Diabetes & $-0.012(0.20)$ & $-0.40 ; 0.38,0.954$ \\
\hline COPD & $0.041(0.33)$ & $-0.61 ; 0.69,0.899$ \\
\hline CAD & $0.026(0.13)$ & $-0.19 ; 0.25,0.814$ \\
\hline Heart failure & $-0.141(0.10)$ & $-0.33 ; 0.05,0.154$ \\
\hline Peripheral vascular disease & $0.132(0.17)$ & $-0.20 ; 0.46,0.436$ \\
\hline Immunocompromised & $-0.002(0.37)$ & $-0.73 ; 0.72,0.995$ \\
\hline Liver disease & $0.103(0.44)$ & $-0.76 ; 0.97,0.815$ \\
\hline Malignancy & $0.009(0.14)$ & $-0.27 ; 0.29,0.945$ \\
\hline CKD & $0.112(0.13)$ & $-0.14 ; 0.37,0.389$ \\
\hline \multicolumn{3}{|l|}{ Acute comorbidities } \\
\hline Sepsis & $0.007(0.18)$ & $-0.34 ; 0.35,0.967$ \\
\hline IMV & $0.028(0.24)$ & $-0.44 ; 0.50,0.907$ \\
\hline NIMV & $-0.029(0.16)$ & $-0.34 ; 0.29,0.857$ \\
\hline Oliguria & $0.067(0.10)$ & $-0.13 ; 0.26,0.508$ \\
\hline Vasoactive drugs & $0.180(0.24)$ & $-0.28 ; 0.64,0.443$ \\
\hline RRT & $-0.110(0.23)$ & $-0.28 ; 0.64,0.443$ \\
\hline Shock & $-0.087(0.44)$ & $-0.96 ; 0.78,0.844$ \\
\hline Major bleeding & $0.167(0.48)$ & $-0.78 ; 1.11,0.729$ \\
\hline APACHE II & $0.004(0.02)$ & $-0.02 ; 0.03,0.795$ \\
\hline \multicolumn{3}{|l|}{ Biochemical markers } \\
\hline CRP & $-0.007(0.00)$ & $-0.003 ; 0.002,0.564$ \\
\hline Serum albumin & $0.100(0.12)$ & $-0.14 ; 0.34,0.406$ \\
\hline
\end{tabular}

Values expressed as mean $( \pm S E)$. CAD, coronary artery disease; COPD, chronic obstructive pulmonary disease; CKD, chronic kidney disease; CRP, C-reactive protein; IMV, invasive mechanical ventilation; NIMV, non-invasive mechanical ventilation. 
Figure 2. Probability of discharge (dot green line) and of transferral to rehabilitation unit (solid red line) according to the degree of muscle wasting (x-axis). The degree of muscle wasting is expressed as standard deviation unit from the mean, in which a negative number indicates higher muscle wasting, a positive number lower muscle wasting. The different shape of the relation between the dot green line and the solid red line was statistically significant $(\mathrm{P}=0.031)$. The probability of discharge was based on multinomial logistic model (mortality is not plotted because only five patients died). The independent variable of the multinomial logistic model was the degree of muscle wasting which was estimated by the mixed models (see text).

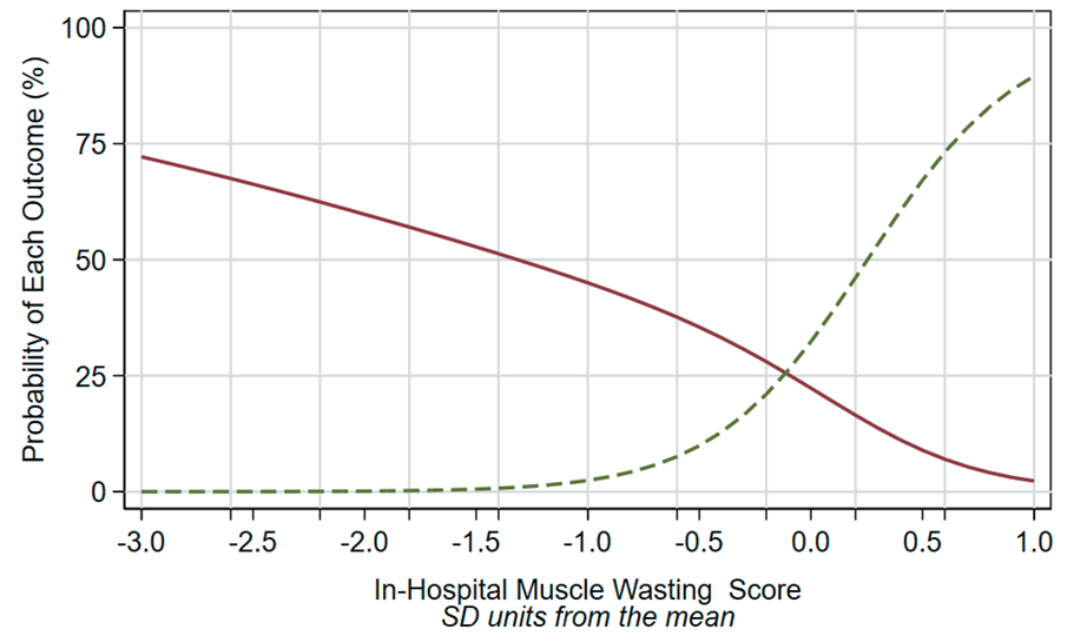

Figure

Transferral ----- Discharge

\section{DISCUSSION}

Our study confirms that muscle loss may occur early and rapidly in the first 5 days of ICU stay, and extends previous observations in general ICU patients also to patients with AKI. At baseline, values of muscle thickness were not different from those of younger healthy subjects when the analysis were adjusted for age and sex. They were also similar to values previously reported in another study assessing elderly healthy subjects (9). However, at day 5, muscle mass was significantly reduced, both in comparison to baseline values and in comparison to the control group.

In our study, the amount of muscle loss was similar to that observed in critically ill patients (8); in particular, at day 5, the VI muscle had the most important reduction in comparison to the RF muscle. Rectus femoris is often described as a power muscle designed to assist in fast movements, while VI is considered a stabilizing muscle that is important for maintaining posture. The identification of which muscles are more affected by immobilization and critical illness in ICU patients could provide important indications in order to more precisely target rehabilitation according to the type of muscle predominantly affected (VI-postural or RF-power). 
We demonstrated that an inverse relationship exists between the severity of muscle loss and the probability of discharge home. Although we can't ascertain the causal relationship between muscle loss and outcome due to the small sample size, baseline sarcopenia assessed by US predicts adverse discharge disposition (death/transferal to nursing facilities) (10). In addition, muscle loss during ICU stay is a major contributor to functional disability (11). However, despite the rapidly loss faced during the first week of ICU stay, it remains unclear whether it is the change in muscle size from baseline or the total amount of muscle mass at admission that is the most important predictor of functional outcome and mortality (12). In our study, baseline muscle thickness did not correlate to the amount of muscle loss, which is in accordance to previous studies investigating muscle wasting in critically ill patients (11).

Despite its increasingly recognized importance, the assessment of muscle mass is challenging in the ICU setting, especially when inflammation and fluid imbalance are present. Currently available bedside methods (such as anthropometry and bioimpedance spectroscopy (BIS)) have important intrinsic limitations, due for example to fluid shifts typical of critically ill patients with AKI (13). Current recommended reference methods are hardly feasible in this clinical setting. Dual energy X-Ray absorptiometry (DEXA), which has been used and recommended by recent Consensus on sarcopenia to assess appendicular skeletal muscle mass (14), is also influenced by hydration status, because it assumes that lean body mass has a constant hydration of 73\%, which is not the case in critically ill patients with AKI (15). In addition, DEXA is not feasible at the bedside and involves patient radiation exposure. Computed tomography (CT) has been recently used to assess muscle mass and its correlation with mortality in critically ill patients (16). Despite its excellent accuracy, CT is expensive, requires specialized personnel, and is available for muscle mass assessment only when CT is necessary for other diagnostic procedures on the lung or the abdomen. In recent years, muscle US has been increasingly studied in the kidney patient setting. Specifically, the reliability of the muscle US technique applied in the present study has been already reported in critically ill patients with AKI, along with excellent intraclass correlation coefficient (ICC) for inter and intra-operator comparisons (3). This methodology has already been validated in patients with AKI against muscle CT (17). Despite the lack of reference values to be applied at baseline and identify patients with pre-ICU low muscle mass and, therefore, increased nutritional risk, in the present study we showed that quadriceps muscle US is useful to monitor nutritional status of critically ill patients with AKI, and that increased muscle loss reduces the chance of being discharged home.

The main limitation of the present study relies in the small sample size, that does not allow further analysis regarding the effect of muscle loss and baseline muscle assets on mortality or functional outcomes. Further studies will be needed on larger cohorts of patients with AKI to allow for such analyses.

In conclusion, muscle wasting occurs early and rapidly within the first 5 days of ICU stay in critically ill patients with AKI. Muscle US is a sensible and feasible tool for the detection 
of muscle wasting even in this clinical setting; moreover, it is easy to use, cheap and timeefficient. In the future, studies defining cut-off values for muscularity are needed to allow the early identification of patients with low muscle mass at ICU admission.

\section{STATEMENT OF AUTHORSHIP:}

Alice Sabatino: Investigation, Methodology, Validation, Writing - original draft. Umberto Maggiore: Formal analysis, Writing - review \& editing. Giuseppe Regolisti: Writing - review \& editing. Giovanni Maria Rossi: Writing - review \& editing. Francesca Di Mario: Resources, Writing - review \& editing. Micaela Gentile: Writing - review \& editing. Maria Teresa Farina: Writing - review \& editing. Enrico Fiaccadori: Supervision, Writing - review \& editing.

Conflict of interest statement: Authors declare that they have no conflict of interest.

Funding sources: This research did not receive any specific grant from funding agencies in the public, commercial, or not-for-profit sectors. 


\section{REFERENCES OF CHAPTER 5}

1. NA A, O’Brien JM, Jr., Hoffmann SP, Phillips G, Garland A, Finley JC, et al. Acquired weakness, handgrip strength, and mortality in critically ill patients. Am J Respir Crit Care Med. 2008;178(3):261-8.

2. Buckinx F, Landi F, Cesari M, Fielding RA, Visser M, Engelke K, et al. Pitfalls in the measurement of muscle mass: a need for a reference standard. J Cachexia Sarcopenia Muscle. 2018;9(2):269-78.

3. Sabatino A, Regolisti G, Bozzoli L, Fani F, Antoniotti R, Maggiore U, et al. Reliability of bedside ultrasound for measurement of quadriceps muscle thickness in critically ill patients with acute kidney injury. Clin Nutr. 2017;36(6):1710-5.

4. Sabatino A, Regolisti G, di Mario F, Ciuni A, Palumbo A, Peyronel F, et al. Validation by CT Scan of Quadriceps Muscle Thickness Measurement by Ultrasound in Acute Kidney Injury. J Nephrol. 2020;33(1):109-17.

5. Kidney Disease Improving Global Outcomes (KDIGO) Acute Kidney Injury Work Group. KDIGO Clinical Practice Guideline for Acute Kidney Injury. Kidney Int. 2012;2:1-138.

6. Sabatino A, Regolisti G, Delsante M, Di Motta T, Cantarelli C, Pioli S, et al. Noninvasive evaluation of muscle mass by ultrasonography of quadriceps femoris muscle in End-Stage Renal Disease patients on hemodialysis. Clin Nutr. 2019;38(3):1232-9.

7. Tillquist M, Kutsogiannis DJ, Wischmeyer PE, Kummerlen C, Leung R, Stollery D, et al. Bedside ultrasound is a practical and reliable measurement tool for assessing quadriceps muscle layer thickness. JPEN J Parenter Enteral Nutr. 2014;38(7):886-90.

8. Parry SM, El-Ansary D, Cartwright MS, Sarwal A, Berney S, Koopman R, et al. Ultrasonography in the intensive care setting can be used to detect changes in the quality and quantity of muscle and is related to muscle strength and function. J Crit Care. 2015;30(5):1151 e9-14.

9. Baldwin $\mathrm{CE}$, Bersten AD. Alterations in respiratory and limb muscle strength and size in patients with sepsis who are mechanically ventilated. Physical therapy. 2014;94(1):68-82.

10. Mueller N, Murthy S, Tainter CR, Lee J, Riddell K, Fintelmann FJ, et al. Can Sarcopenia Quantified by Ultrasound of the Rectus Femoris Muscle Predict Adverse Outcome of Surgical Intensive Care Unit Patients as well as Frailty? A Prospective, Observational Cohort Study. Annals of surgery. 2016;264(6):1116-24.

11. Puthucheary Z, Rawal J, McPhail M, Connolly B, Ratnayake G, Chan P, et al. Acute Skeletal Muscle Wasting in Critical Illness. Jama. 2013;310(15).

12. Puthucheary ZA, Hart N. Skeletal muscle mass and mortality - but what about functional outcome? Crit Care. 2014;18(1):110.

13. Carrero JJ, Johansen KL, Lindholm B, Stenvinkel P, Cuppari L, Avesani CM. Screening for muscle wasting and dysfunction in patients with chronic kidney disease. Kidney Int. 2016;90(1):53-66.

14. Bauer J, Morley JE, Schols A, Ferrucci L, Cruz-Jentoft AJ, Dent E, et al. Sarcopenia: A Time for Action. An SCWD Position Paper. J Cachexia Sarcopenia Muscle. 2019;10(5):956-61. 
15. Pietrobelli A, Formica C, Wang Z, Heymsfield SB. Dual-energy X-ray absorptiometry body composition model: review of physical concepts. The American journal of physiology. 1996;271(6 Pt 1):E941-51.

16. Akkoc I, Toptas M, Yalcin M, Demir E, Toptas Y. Psoas Muscle Area Measured with Computed Tomography at Admission to Intensive Care Unit: Prediction of In-Hospital Mortality in Patients with Pulmonary Embolism. Biomed Res Int. 2020;2020:1586707.

17. Sabatino A RG, di Mario F, Ciuni A, Palumbo A, Peyronel F, Maggiore U, Fiaccadori E. Validation by CT scan of quadriceps muscle ultrasound in acute kidney injury. J Nephrol. 2019; Epub ahead of print. 



\section{CHAPTER 6}

Quadriceps muscle thickness

assessed by ultrasound

outperforms conventional

anthropometry in the evaluation

of mortality risk in hemodialysis

patients

Sabatino A, Kooman J, Di Motta T, Cantarelli C, Gregorini MC, Bian-

chi S, Farina MT, Di mario F, Regolisti G, Fiaccadori E 


\section{ABSTRACT}

Background: Muscle ultrasound (US) is a valid and reliable tool to assess skeletal muscle dimensions in patients on chronic hemodialysis (HD), however it has not been previously related to outcomes in this population. In the present study we assess the relation between dimensions derived from muscle US and outcomes in ESKD patients on HD in comparison to anthropometry.

Methods: 181 prevalent HD patients were prospectively evaluated. Quadriceps rectus femoris (QRFT) and vastus intermedius thickness (QVIT) were assessed using B-mode US, and were indexed for height. Mid-arm muscle circumference (MAMC) and area (MAMA) were assessed by anthropometry, and were also indexed for height. Median follow up time was 35 months. Kaplan-Meier and Cox regression analysis were used to assess the relation of gender-based percentiles (p) of US and anthropometric parameters with mortality.

Results: Unlike indexed MAMC and MAMA, both proximal as well as distal indexed QRFT and QVIT were significantly lower in patients who died as compared to patients who were alive at the end of the follow up period. The AUC values for the prediction of mortality were statistically significant at p25 and p50 US derived measurements, but not for anthropometry. In the adjusted Cox-regression analysis, the indexed MAMC and indexed distal QRFT and QVIT were independently related to mortality at p25 and p50.

Conclusion: Indexed QRFT and QVIT independently predicted mortality in patients on HD, and outperformed anthropometry in this respect. Muscle US is a simple practical tool that adds prognostic information to the bedside nutritional assessment in ESKD patients on maintenance HD.

Key words: end-stage kidney disease; hemodialysis; mortality; muscle mass; ultrasound 


\section{INTRODUCTION}

End-stage kidney disease (ESKD) is a catabolic condition characterized also by reduced protein synthesis (1). This imbalance between catabolism and anabolism results in skeletal muscle loss. In fact, despite muscle loss being also dependent on physiological ageing, it occurs earlier and more rapidly in patients on hemodialysis in comparison to age-matched controls $(2,3)$. Reasons for this increase in catabolism are related to three main contributors, namely, the metabolic consequences of the loss of kidney function, the lifesaving kidney replacement therapy, and the presence of many other chronic comorbidities associated with negative impact on nutritional status (1).

Skeletal muscle is vital to mobility, posture, strength and balance, allowing for routine physical activities and exercise (4). In addition, it is also a pivotal metabolic and homeostatic organ (5). Most importantly, it plays a key role in protein metabolism as a source of amino acids when protein intake is insufficient, thus preserving the protein content of other essential organs $(5,6)$. However, when muscles undergo chronic catabolism to supply amino acids for other metabolic purposes, without parallel activation of a compensatory increased protein synthesis, a reduction in muscle mass is observed. This very fact has potentially serious clinical consequences, such as muscle weakness, impaired physical function, increased morbidity and mortality (7).

Consequently, an important part of the routine nutritional evaluation of patients on chronic $\mathrm{HD}$ is represented by the assessment of body composition, which typically refers to the quantification of adipose tissue and muscle mass (8). Since the identification of reduced muscle mass is critical, a precise and accurate method that is also economically viable should be the preferred approach to diagnose muscle wasting, aiming for an early diagnosis and monitoring in patients at risk of muscle loss. In this regard, the use of ultrasound (US) for the assessment of muscle dimensions has received considerable interest in recent years. In fact, this diagnostic technique can be performed easily at the bedside even in non-collaborative patients, it is economically viable, safe and does not require specialized staff or X-ray exposure (9-11). The reliability of US in assessing quadriceps muscle thickness has recently been documented both in critically ill patients with AKI (10), in whom it has also been validated against CT (12), and in ESKD patients on HD (11). Muscle US is not affected by alterations in fluid status $(10,11)$, which are very common in this clinical setting and one of the principal problems in applying currently available bedside techniques (13). However, the ability of this method to predict mortality has not been studied in the renal patient setting. Moreover, since the technique has been utilized only recently for body composition assessment, reference values are lacking. With this background, in the present study we aimed to evaluate the performance of quadriceps muscle thickness, as assessed by US, in predicting mortality in patients on maintenance HD. In addition, we derived possible cut-offs of muscle thickness that could predict mortality, and compared them with those derived from the conventional anthropometric assessment. 


\section{MATERIALS AND METHODS}

\section{STUDY DESIGN AND PATIENTS}

This is an ongoing observational prospective study of 181 prevalent patients on maintenance HD from 6 dialysis centers in northern and central Italy (3 in Parma, 1 in Reggio Emilia and 2 in Livorno). The study was approved by the local Institutional Review Board (AVEN ref. $n^{\circ} 45737$, December 12th, 2015). The procedures were in agreement with the Declaration of Helsinki, and written informed consent was obtained from all participants. We referred to the STROBE checklist to report our results. Patients were evaluated between January 2016 and March 2018 and followed for mortality events until April 2020, for a median of 35 months (interquartile range, 23-41 months). Patients were censored if received kidney transplant or transferred to another center. The main objective of this cohort study was to assess the correlation between muscle thickness by ultrasound and other nutritional status evaluation parameters (11). Only adult patients ( $\geq 18$ years of age) with a dialysis vintage of at least 6 months were enrolled. Patients with malignancy or with conditions associated with mandatory immobilization were excluded.

\section{ULTRASOUND TECHNIQUE}

Quadriceps rectus femoris and vastus intermedius thickness (QRFT and QVIT) were measured by experienced assessors using B-mode ultrasonography with a wall-tracking ultrasound system (Philips hd7xe, Logiq and General electric) and 7.5 MHz linear array transducers. The technique used has already been described in detail previously $(10,11)$. Briefly, measurements were performed at two landmarks for each leg with the patient lying in a supine position with both knees extended but relaxed and toes pointing to the ceiling, during the HD session. Quadriceps rectus femoris and vastus intermedius thickness (QRFT; QVIT) were measured at the border between the upper third (QRFT Prox; QVIT Prox) and lower two-thirds (QRFT Dist; QVIT Dist) between the anterior superior iliac spine (ASIS) and the upper pole of the patella $(10,14)$. The transducer was placed perpendicular to the long axis of the thigh with a large amount of gel and with no pressure to avoid compression of the muscle. The vertical diameter of the muscles was measured at the widest point, on the inner edge of the muscle fascia. Measurements were performed directly on line while obtaining the images. Three experienced assessors (one for the centers in Parma and Reggio Emilia, 1 for each center from Livorno), all of whom had received previously proper training on the described method, performed all measurements. Two measurements on both legs for each site were averaged and were used for the analysis (a total of 4 values for each subject). Previously published data shows that fluid overload do not interfere with quadriceps muscle US $(10,11)$, and to allow standardization of the protocol, all measurements were performed before the hemodialysis session. For further analyses, measurements were normalized by height squared.

\section{ANTHROPOMETRICS}

Anthropometric measurements included body weight, height, mid-arm circumference (MAC) and triceps skinfold (TSKF) thickness assessed after the dialysis session. Body weight (Kg) 
was assessed by electronic bed scales and height $(\mathrm{m})$ using a stadiometer. TSKF $(\mathrm{mm})$ was measured using the Lange Skinfold Caliper (Cambridge Scientific Industries Inc., Cambridge, $M D, U S A)$ and MAC was assessed by a non-stretchable measure tape. Body mass index (BMI) was calculated as body weight (Kg) divided by square height (m2), mid-arm muscle circumference (MAMC) and mid-arm muscle area (MAMA) were normalized by height squared (indexed MAMC and MAMA) and were calculated with the following equations:

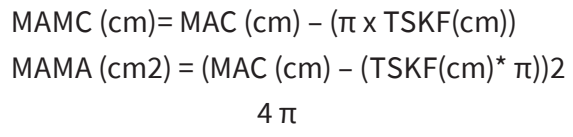

\section{BIOCHEMICAL MEASUREMENTS}

Blood samples were collected pre-dialysis as per institutional routine, preferably on a midweek dialysis day, for assessment of serum creatinine, blood urea nitrogen (BUN), phosphate, PTH, albumin, and C-reactive protein (CRP).

\section{STATISTICAL ANALYSIS}

Results are expressed as mean and standard deviation for continuous variables with normal distribution, or median and range for non-parametric data, and as frequencies for categorical variables. Group differences were analyzed using Student $t$ test and Mann-Whitney's U test for parametric and non-parametric data, respectively to assess difference between means of deceased and living patients. Muscle thickness assessed by US was distributed in percentiles based on gender. Receiver operating characteristic (ROC) curve analysis was applied for p10, p25 and p50 whereby the area under the curve (AUC) indicated the probability of measures to identify patients at risk of mortality. The same analysis was used for indexed MAMC and MAMA. KaplanMeier survival analysis and Cox proportional-hazards regression analysis were performed applying the derived cut-offs for muscle dimensions. Cox proportional-hazards regression analysis was adjusted for confounding variables identified by univariate analysis (age, serum albumin, serum CRP, diabetes, COPD, cardiovascular diseases, heart failure). All analyses were performed using IBM Statistical Package for Social Sciences version 26.0 (IBM SPSS Statistics Inc. Chicago IL. USA). Statistical significance was set at $p<0.05$ for all tested parameters.

\section{RESULTS}

\section{CLINICAL AND DEMOGRAPHIC CHARACTERISTICS OF ENROLLED PATIENTS}

We enrolled 181 patients, (123 males (68\%), mean age $65 \pm 16$ years) (Table 1). Males and females differed by dry body weight and height (but not BMI), blood urea nitrogen (BUN), 
prevalence of chronic obstructive pulmonary disease, peripheral and cerebral vascular disease, TSKF thickness, indexed MAMC and MAMA and all absolute values of quadriceps muscle thickness (Table 1). However, when muscle thickness was normalized by height squared (QRFT and QVIT index), no difference was found between groups.

Table 1. Demographic and clinical characteristics of the entire cohort.

\begin{tabular}{|c|c|c|c|}
\hline & Overall $(n=181)$ & Male $(n=123)$ & Female $(n=58)$ \\
\hline Age (years) & $65(16)$ & $66(17)$ & $64(16)$ \\
\hline Sex (\%) & $123 / 181(68)$ & - & - \\
\hline $\begin{array}{l}\text { Time in study (median; interquartile range) } \\
\text { (months) }\end{array}$ & $35(22 ; 41)$ & $34(20 ; 41)$ & $36(26 ; 41)$ \\
\hline $\begin{array}{l}\text { HD vintage (median; interquartile range) } \\
\text { (months) }\end{array}$ & $55(17 ; 96)$ & $57(17 ; 97)$ & $50(17 ; 96)$ \\
\hline Body weight (Kg) & $68.6(12.8)$ & $71.2(12.0)$ & $63(13)^{\star \star \star}$ \\
\hline Height (cm) & $166(8.7)$ & $170(6.6)$ & $157(6.3)^{\star \star \star}$ \\
\hline $\mathrm{BMI}(\mathrm{Kg} / \mathrm{m} 2)$ & $24.8(4.5)$ & $24.6(4.2)$ & $25.3(5.1)$ \\
\hline \multicolumn{4}{|l|}{ Biochemical data } \\
\hline $\mathrm{sCr}(\mathrm{mg} / \mathrm{dl})$ & $8.7(3.1)$ & $9.0(3.3)$ & $8.2(2.5)$ \\
\hline BUN (mg/dl) & $70.1(18.7)$ & $67.9(16.5)$ & $74.8(22.1)^{\star}$ \\
\hline$P(m g / d l)$ & $5.2(1.6)$ & $5.2(1.7)$ & $5.3(1.5)$ \\
\hline PTH (pg/ml) & $208(98 ; 365)$ & $192(103 ; 340)$ & $242(77 ; 459)$ \\
\hline Albumin (g/dl) & $3.7(0.4)$ & $3.7(0.4)$ & $3.6(0.3)$ \\
\hline $\mathrm{CRP}(\mathrm{mg} / \mathrm{l})$ & $0.87(0.26 ; 2.75)$ & $0.95(0.95 ; 3)$ & $0.71(0.22 ; 2.36)$ \\
\hline \multicolumn{4}{|l|}{ Comorbidities } \\
\hline Diabetes mellitus (\%) & $51 / 181(28)$ & $36 / 123(29)$ & $15 / 58(26)$ \\
\hline COPD (\%) & $15 / 181(8)$ & $14 / 123(11)$ & $1 / 58(2)^{\star}$ \\
\hline Cardiovascular diseases (\%) & $77 / 181(43)$ & $57 / 123(46)$ & $20 / 58(34)$ \\
\hline Heart failure (\%) & $21 / 181(12)$ & $16 / 123(13)$ & $5 / 58(9)$ \\
\hline \multicolumn{4}{|l|}{ Anthropometry and muscle US } \\
\hline Triceps SKF (mm) & $13.1(6)$ & $11.7(5.3)$ & $16.1(6.2)^{\star \star \star}$ \\
\hline $\operatorname{MAC}(\mathrm{cm})$ & $28.5(4.1)$ & $28.1(3.8)$ & $29.2(4.8)$ \\
\hline MAMC (cm) & $24.4(3.2)$ & $24.5(3.1)$ & $24.2(3.4)$ \\
\hline MAMC index (cm/height2) & $8.9(1.6)$ & $8.5(1.3)$ & $9.8(1.7)^{\star \star \star}$ \\
\hline MAMA (cm2) & $48.2(12.8)$ & $48.6(12.2)$ & $47.4(14.1)$ \\
\hline MAMA index (cm2/height2) & $17.6(5.3)$ & $16.8(4.6)$ & $19.2(6.1)^{\star}$ \\
\hline QRFT Prox (mm) & $16.1(4.4)$ & $16.8(4.5)$ & $14.4(3.7)^{\star \star \star}$ \\
\hline QVIT Prox (mm) & $13.1(4.8)$ & $13.9(5.3)$ & $11.5(3.0)^{\star \star}$ \\
\hline QRFT Dist (mm) & $10.9(3.6)$ & $11.4(3.8)$ & $9.8(2.9)^{\star \star}$ \\
\hline QVIT Dist (mm) & $10.1(3.4)$ & $10.6(3.7)$ & $8.8(2.4)^{\star \star \star}$ \\
\hline
\end{tabular}


Table 1. Continued

\begin{tabular}{llll} 
& Overall $(\mathbf{n}=\mathbf{1 8 1})$ & Male $(\mathbf{n}=\mathbf{1 2 3})$ & Female $(\mathbf{n}=\mathbf{5 8})$ \\
\hline QRFT Prox index (mm/height2) & $5.8(1.6)$ & $5.8(1.6)$ & $5.7(1.4)$ \\
\hline QVIT Prox index (mm/height2) & $4.8(1.7)$ & $4.8(1.8)$ & $4.7(1.3)$ \\
\hline QRFT Dist index (mm/height2) & $3.9(1.3)$ & $4.0(1.3)$ & $3.9(1.1)$ \\
\hline QVIT Dist index (mm/height2) & $3.6(1.2)$ & $3.7(1.3)$ & $3.5(1.0)$ \\
\hline Deceased $(\%)$ & $66 / 181(36)$ & $49 / 123(40)$ & $17 / 58(29)$
\end{tabular}

${ }^{\star \star *} \mathrm{P}<0.0001 ;{ }^{\star \star} \mathrm{P}<0.01 ;{ }^{*} \mathrm{P}<0.05$, female versus male patients. Data are expressed as mean (SD), median (interquartile range) and frequency (\%). BMI, body mass index; BUN, blood urea nitrogen; CAD, coronary arterial disease; COPD, chronic obstructive pulmonary disease; CRP, C-reactive protein; DM, diabetes mellitus; HD, hemodialysis; MAC, mid-arm circumference; MAMA, mid-arm muscle area; MAMC, mid-arm muscle circumference; PTH, parathyroid hormone; QRFT, quadriceps rectus femoris thickness; QVIT, quadriceps vastus intermedius thickness.

\section{COMPARISON BETWEEN DECEASED AND ALIVE PATIENTS}

Overall, patients were followed for a median of 35 months (interquartile range, 22 to 41 months). During this period, 36\% (66/181) died. Patients who died were older, had lower serum albumin, and higher serum CRP values. In addition, they had higher prevalence of comorbidities and lower muscle thickness as assessed by US (Table 2 and Figure 1). Conversely, MAMC index and MAMA index were not different between groups (Figure 1). However, TSKF thickness, an index of fat mass, was lower in deceased patients.

Table 2. Demographic and clinical characteristics according to mortality status

\begin{tabular}{|c|c|c|c|}
\hline & Deceased $(n=66)$ & Alive $(n=115)$ & $\mathbf{P}$ \\
\hline Age (years) & $73.7(12.6)$ & $60.8(16.7)$ & $<0.0001$ \\
\hline Male sex (\%) & $49 / 66(74)$ & $74 / 115(64)$ & 0.19 \\
\hline HD vintage (median; interquartile range) (month) & $60(29 ; 109)$ & $50(16 ; 95)$ & 0.16 \\
\hline $\mathrm{Kt} / \mathrm{V}^{*}$ & $1.48(0.28)$ & $1.49(0.29)$ & 0.799 \\
\hline $\mathrm{BMI}(\mathrm{Kg} / \mathrm{m} 2)$ & $24.5(4.1)$ & $25.0(4.7)$ & 0.48 \\
\hline Albumin (g/dl) & $3.6(0.4)$ & $3.8(0.4)$ & $<0.0001$ \\
\hline $\mathrm{CRP}(\mathrm{mg} / \mathrm{l})$ & $1.36(0.51 ; 3.35)$ & $0.79(0.19 ; 2.34)$ & 0.02 \\
\hline \multicolumn{4}{|l|}{ Comorbidities } \\
\hline Diabetes mellitus (\%) & $27 / 66(41)$ & $24 / 115(21)$ & 0.006 \\
\hline COPD (\%) & $11 / 66(17)$ & $4 / 115(3.5)$ & 0.004 \\
\hline Cardiovascular diseases (\%) & $41 / 66(62)$ & $36 / 115(31)$ & $<0.0001$ \\
\hline Heart failure (\%) & $14 / 66(21)$ & $7 / 115(6)$ & 0.003 \\
\hline \multicolumn{4}{|l|}{ Anthropometry and muscle US } \\
\hline TSKF (mm) & $11.6(6.5)$ & $14.0(5.5)$ & 0.008 \\
\hline MAC (cm) & $27.7(4.1)$ & $28.9(4.1)$ & 0.04 \\
\hline
\end{tabular}


Table 2. Continued

\begin{tabular}{llll} 
& Deceased $(\mathbf{n}=\mathbf{6 6})$ & Alive $(\mathbf{n}=\mathbf{1 1 5})$ & P \\
\hline MAMC $(\mathrm{cm})$ & $24.0(3.0)$ & $24.6(3.4)$ & 0.27 \\
\hline MAMC index $\left(\mathrm{cm} /\right.$ height $\left.^{2}\right)$ & $8.7(1.6)$ & $8.9(1.5)$ & 0.43 \\
\hline MAMA $(\mathrm{cm} 2)$ & $46.6(11.3)$ & $49.1(13.6)$ & 0.21 \\
\hline MAMA index $\left(\mathrm{cm} /\right.$ height $\left.^{2}\right)$ & $16.9(4.8)$ & $18.0(5.5)$ & 0.28 \\
\hline QRFT Prox index $(\mathrm{mm} /$ height2) & $5.3(1.5)$ & $6.1(1.5)$ & 0.0007 \\
\hline QVIT Prox index $(\mathrm{mm} /$ height2) & $4.2(1.3)$ & $5.1(1.8)$ & 0.0003 \\
\hline QRFT Dist index $(\mathrm{mm} /$ height2) & $3.5(1.2)$ & $4.2(1.3)$ & 0.0004 \\
\hline QVIT Dist index $(\mathrm{mm} /$ height2) & $3.2(1.1)$ & $3.9(1.2)$ & 0.001
\end{tabular}

${ }^{\star}$ Available for 115 patients. Data expressed as mean (SD), median (interquartile range) and frequency (\%). BMI, body mass index; COPD, chronic obstructive pulmonary disease; CRP, C-reactive protein; Dist, distal; DM, diabetes mellitus; HD, hemodialysis; MAC, mid-arm circumference; MAMA, mid-arm muscle area; MAMC, mid-arm muscle circumference; PTH, parathormone; Prox, proximal; QRFT, quadriceps rectus femoris thickness; QVIT, quadriceps vastus intermedius thickness; TSKF, triceps skinfold.

Figure 1. Box-plot of the differences in muscle dimensions assessed by ultrasound and anthropometry between deceased and living patients.

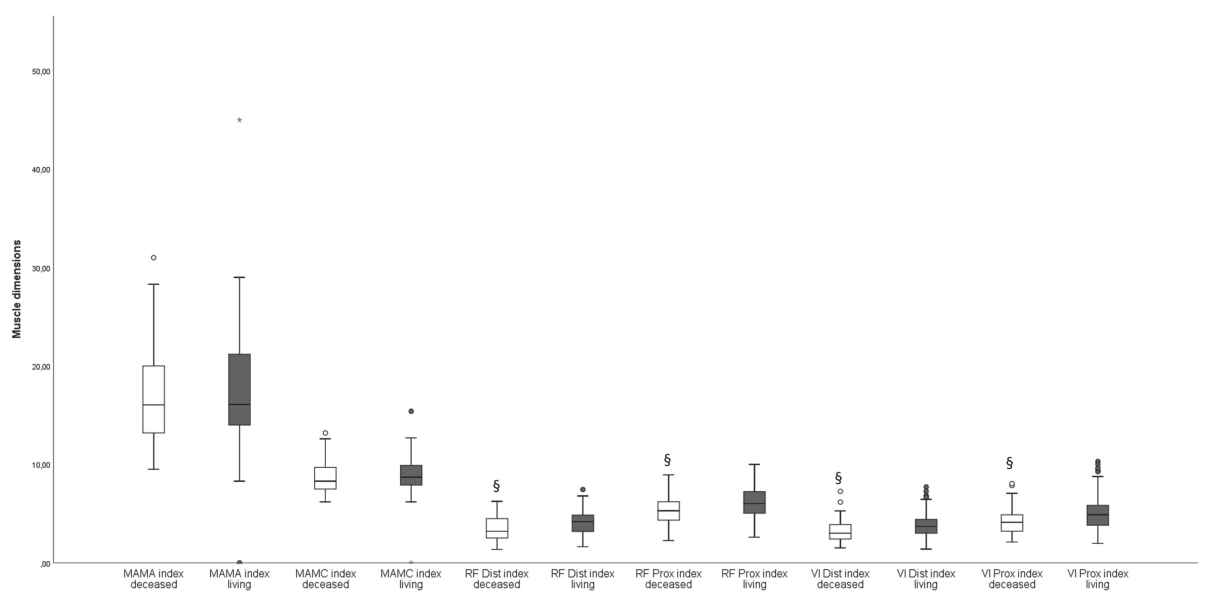

Legend. $\S \mathrm{P}<0.001$. MAMC, Mid-arm muscle circumference; MAMA, Mid-arm muscle area; RF, Rectus femoris, VI, Vastus intermedius.

\section{ASSOCIATION OF MUSCLE DIMENSIONS AS ASSESSED BY US AND ANTHROPOMETRY WITH MORTALITY}

Indexed QRFT and QVIT measured at proximal and distal sites differed significantly between deceased and alive patients (Table 2). At univariate analysis, age, serum albumin, serum CRP, presence of diabetes, COPD, cardiovascular diseases and heart failure were also different between groups. 
We used the p10, p25 and p50 values of the gender-stratified distributions of indexed muscle dimensions as assessed by US and anthropometry (for the complete distribution see Supplementary table 1), as the cut-off values to identify patients with low muscle mass, and analyzed its relation with mortality using ROC curves (Table 3). The AUC of indexed MAMC and MAMA was not statistically significant for any percentile, whilst the distal values of indexed QRFT and QVIT were statistically significant at p25 and p50, with p50 showing the highest sensitivity.

Table 3. Area under curve for different cut-offs of muscle dimensions obtained by ultrasound and anthropometry.

\begin{tabular}{|c|c|c|c|}
\hline p10 & AUC $(95 \% \mathrm{CI}), \mathrm{P}$ & Sensitivity & Specificity \\
\hline QRFT Prox index (3.60 male; 3.62 female) & 0.57 (0.48 - 0.66), 0.12 & 18.2 & 95.6 \\
\hline QVIT Prox index (2.76 male; 2.85 female) & $0.56(0.47-0.65), 0.20$ & 16.7 & 94.8 \\
\hline QRFT Dist index (2.20 male; 2.44 female) & $0.57(0.48-0.66) 0.12$ & 18.2 & 95.6 \\
\hline QVIT Dist index (2.28 male; 2.41 female) & $0.54(0.46-0.63), 0.31$ & 15.1 & 93.9 \\
\hline MAMC index (6.9 male; 7.8 female) & $0.51(0.43-0.60), 0.74$ & 9.1 & 93.9 \\
\hline MAMA index (11.6 male; 13.0 female) & 0.50 (0.41-0.59), 0.97 & 9.1 & 91.2 \\
\hline p25 & AUC $(95 \% \mathrm{Cl}), \mathrm{P}$ & Sensitivity & Specificity \\
\hline QRFT Prox index (4.76 male; 4.81 female) & $0.58(0.49-0.67), 0.06$ & 34.8 & 81.7 \\
\hline QVIT Prox index (3.56 male; 3.80 female) & $0.58(0.49-0.67), 0.078$ & 34.9 & 80.9 \\
\hline QRFT Dist index (3.15 male; 2.9 female) & $0.59(0.50-0.68), 0.04$ & 36.4 & 81.7 \\
\hline QVIT Dist index (2.61 male; 2.94 female) & $0.60(0.51-0.69), 0.02$ & 37.9 & 82.6 \\
\hline MAMC index ( 7.50 male; 8.60 female) & 0.55 (0.47- 0.65), 0.19 & 25.8 & 85.9 \\
\hline MAMA index (13.30 male; 14.90 female) & $0.53(0.44-0.62), 0.49$ & 27.3 & 78.9 \\
\hline p50 & AUC $(95 \% \mathrm{Cl}), \mathrm{P}$ & Sensitivity & Specificity \\
\hline QRFT Prox index (5.81 male; 5.77 female) & $0.57(0.48-0.65), 0.139$ & 57.6 & 55.6 \\
\hline QVIT Prox index (4.57 male; 4.80 female) & $0.58(0.49-0.67), 0.069$ & 60.6 & 55.6 \\
\hline QRFT Dist index (3.89 male; 4.02 female) & $0.62(0.53-0.70), 0.009$ & 65.1 & 58.3 \\
\hline QVIT Dist index (3.44 male; 3.50 female) & $0.63(0.54-0.71), 0.005$ & 65.2 & 60.0 \\
\hline MAMC index (8.30 male; 9.30 female) & $0.52(0.43-0.61), 0.66$ & 47.0 & 57.0 \\
\hline MAMA index (16.0 male; 17.20 female) & $0.51(0.42-0.60), 0.79$ & 51.5 & 50.9 \\
\hline
\end{tabular}

AUC, Area under the curve; $\mathrm{Cl}$, Confidence interval; MAMA, Mid-arm muscle area; MAMC, Mid-arm muscle circumference; QRFT, quadriceps rectus femoris thickness; QVIT, quadriceps vastus intermedius thickness. QRFT and QVIT index are presented in $\mathrm{mm} / \mathrm{m}^{2} ;$ MAMC index is presented in $\mathrm{cm} / \mathrm{m}^{2}$, and MAMA index is presented as $\mathrm{cm}^{2} / \mathrm{m}^{2}$

Kaplan-Meier curves were fitted to p10 (Figure 2A-F), p25 (Figure 3A-F) and p50 (Figure 4A-F) indexed values of quadriceps muscle thickness and anthropometric muscle dimensions. Survival curves for indexed QRFT and QVIT were statistically significant for all analyzed percentiles, while only indexed MAMC at p25 was statistically significant. 
Figure 2A-F. Kaplan-Meier survival curves according to reduced muscle dimensions as assessed by ultrasound and anthropometry using $\mathrm{p} 10$ as the reference value.
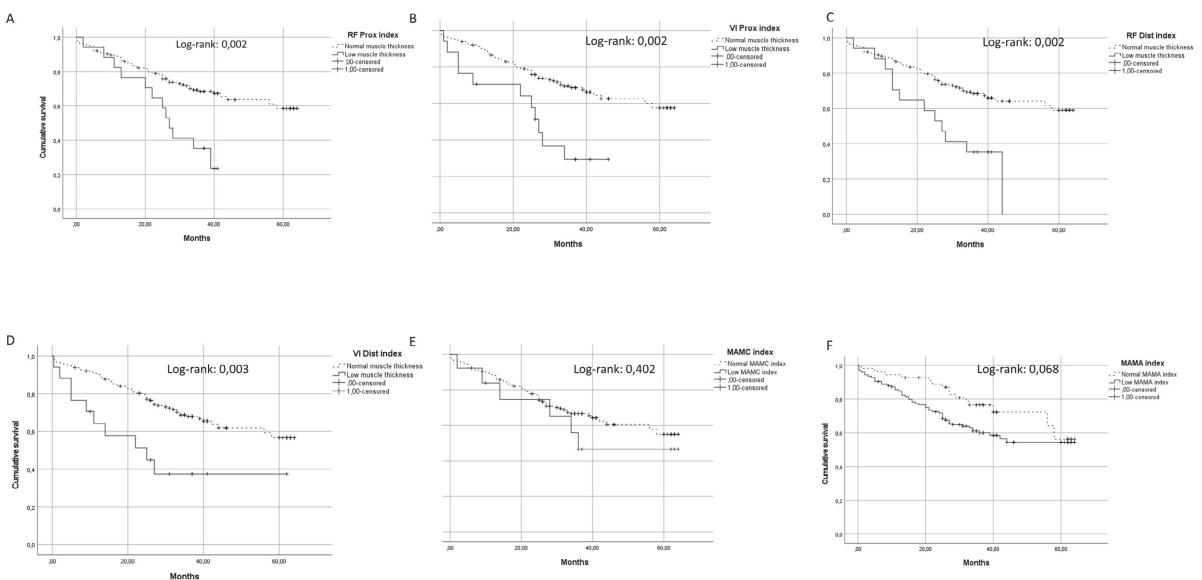

\section{Legend.}

A. $\quad$ Proximal QRFT index (male: 3.6 ; female: $3.62 \mathrm{~mm} / \mathrm{m} 2$ )

B. $\quad$ Proximal QVIT index (male: 2.76 ; female: $2.85 \mathrm{~mm} / \mathrm{m} 2$ )

C. Distal QRFT index (male: 2.20 ; female: $2.44 \mathrm{~mm} / \mathrm{m} 2$ )

D. Distal QVIT index (male: 2.28 ; female: $2.41 \mathrm{~mm} / \mathrm{m} 2$ )

E. $\quad$ MAMC index (male: 6.9 ; female: $7.8 \mathrm{~cm} / \mathrm{m} 2$ )

F. $\quad$ MAMA index (male: 11.6 ; female: $13.0 \mathrm{~cm} 2 / \mathrm{m} 2$ )

MAMC, Mid-arm muscle circumference; MAMA, Mid-arm muscle area; QRFT, quadriceps rectus femoris; QVIT, quadriceps vastus intermedius thickness.

Figure 3A-F. Kaplan-Meier survival curve based on the presence of low muscle dimensions as assessed by ultrasound and anthropometry using p25 as the reference value.
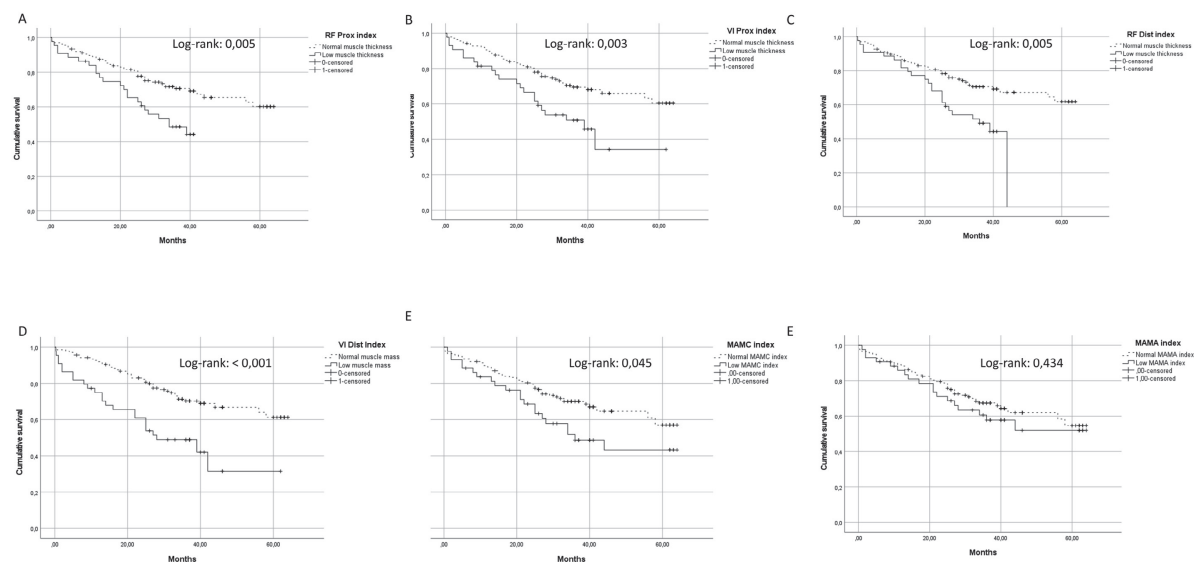
Figure 3A-F. Continued

Legend.
A. $\quad$ Proximal QRFT index (male: 4.76 ; female: $4.81 \mathrm{~mm} / \mathrm{m} 2$ )
B. Proximal QVIT index (male: 3.56 ; female: $3.80 \mathrm{~mm} / \mathrm{m} 2$ )
C. Distal QRFT index (male: 3.15 ; female: $2.90 \mathrm{~mm} / \mathrm{m} 2$ )
D. Distal QVIT index (male: 2.61 ; female: $2.94 \mathrm{~mm} / \mathrm{m} 2$ )
E. $\quad$ MAMC index (male: 7.5 ; female: $8.6 \mathrm{~cm} / \mathrm{m} 2$ )
F. $\quad$ MAMA index (male: 13.3 ; female: $14.9 \mathrm{~cm} 2 / \mathrm{m} 2$ )

MAMC, Mid-arm muscle circumference; MAMA, Mid-arm muscle area; QRFT, quadriceps rectus femoris; QVIT, quadriceps vastus intermedius thickness.

Figure 4A-F. Kaplan-Meier survival curve according to reduced muscle dimensions as assessed by ultrasound and anthropometry using p50 as the reference value.
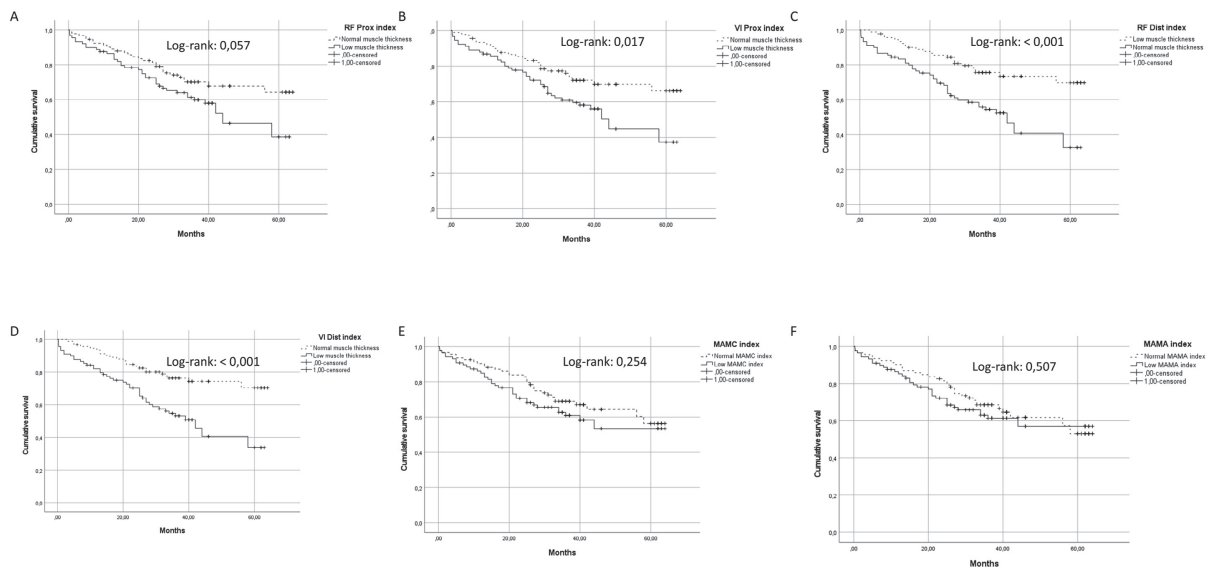

\section{Legend.}
A. $\quad$ Proximal QRFT index (male: 5.81 ; female: $5.77 \mathrm{~mm} / \mathrm{m} 2$ )
B. $\quad$ Proximal QVIT index (male: 4.57 ; female: $4.80 \mathrm{~mm} / \mathrm{m} 2$ )
C. Distal QRFT index (male: 3.89 ; female: $4.02 \mathrm{~mm} / \mathrm{m} 2$ )
D. Distal QVIT index (male: 3.44 ; female: $3.50 \mathrm{~mm} / \mathrm{m} 2$ )
E. $\quad$ MAMC index (male: 8.3 ; female: $9.3 \mathrm{~cm} / \mathrm{m} 2$ )
F. $\quad$ MAMA index (male: 16.0 ; female: $17.2 \mathrm{~cm} 2 / \mathrm{m} 2$ )

MAMC, Mid-arm muscle circumference; MAMA, Mid-arm muscle area; QRFT, quadriceps rectus femoris; QVIT, quadriceps vastus intermedius thickness.

After this, we used Cox proportional-hazards regression analysis to assess the predictive performance of muscle dimensions towards mortality using p10, p25 and p50 values (Table 4). All US parameters were statistically significant at crude analysis, whilst in the fully adjusted model distal QVIT index was an independent predictor of mortality using the p10, p25 and p50 values, and distal QRFT index was still statistically significant only using the p10 and p50 value. As for anthropometric variables, both p25 and p50 values of MAMC index were statistically significant in the fully adjusted model, but not at crude analysis. 


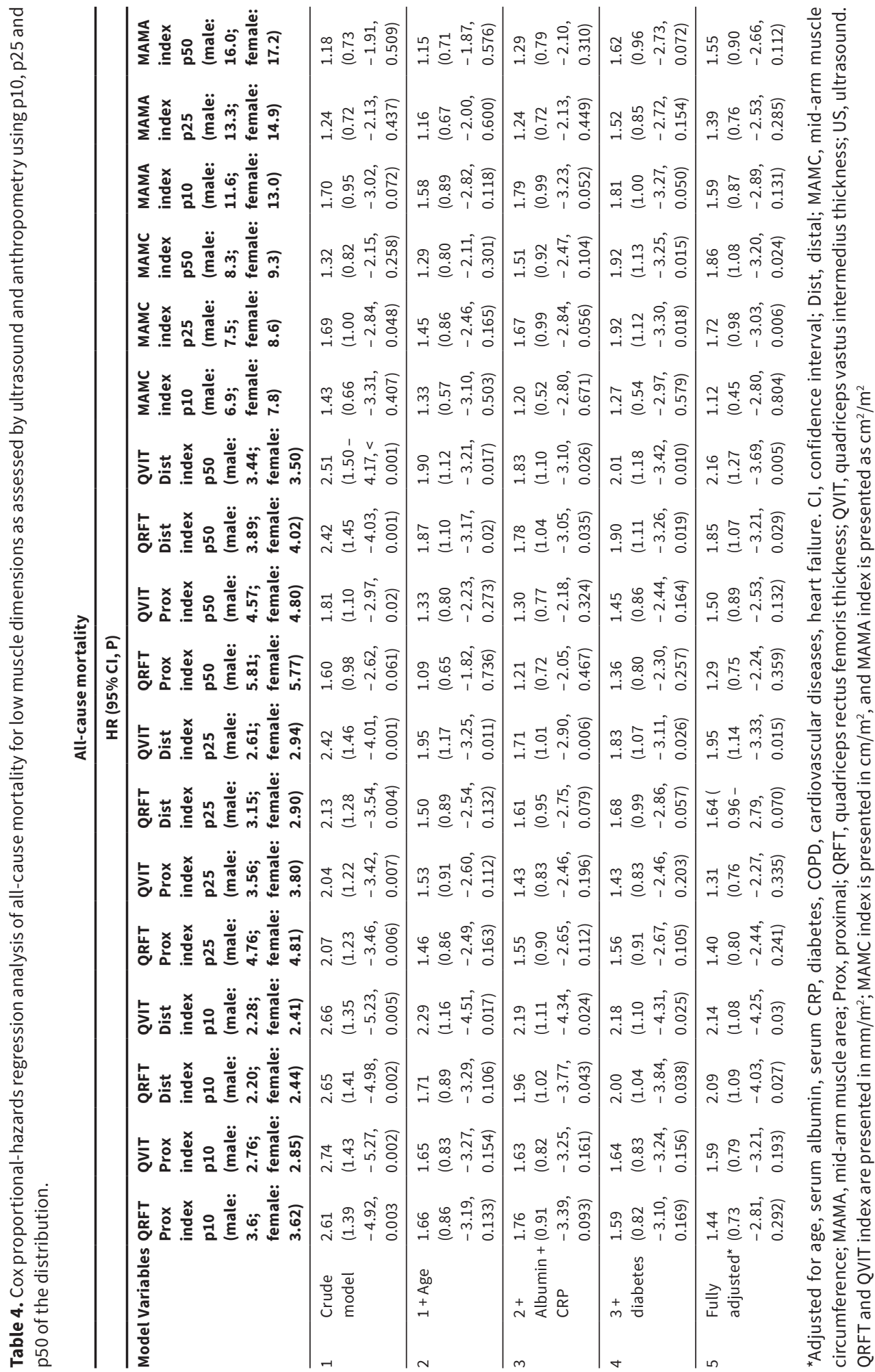




\section{DISCUSSION}

In the present study we report that low indexed quadriceps muscle thickness values, as assessed by US, were independently associated with mortality in patients with ESKD on maintenance HD. In addition, we compared the predictive value of different cut-off values of muscle dimensions obtained by US and anthropometry with respect to mortality.

To our knowledge, this is the first time that muscle US was correlated with mortality risk in ESKD patients on chronic HD, and the first time that US and anthropometry were compared regarding outcome prediction. The predictive of muscle dimensions on outcomes in HD patients has been explored previously in this population, showing that reduced muscle mass as assessed by body composition or surrogate methods increase the risk of worse outcomes (15-20). In fact, muscle wasting is very common in patients with ESKD on HD $(11,21,22)$, as it is a consequence of metabolic acidosis, chronic inflammation, and anorexia related to the kidney disease per se, as well as of renal replacement therapy and comorbidities associated with increased protein catabolism and reduced protein anabolism (1). Despite its recognized importance, the assessment of muscle mass is challenging in renal patients. Current bedside methods (such as anthropometry and bioimpedance spectroscopy (BIS)) have important limitations, both intrinsic to the methods and caused by fluid balance derangements typical of this clinical setting (23).

Traditionally, MAMC has been widely used as a marker of muscle mass in clinical practice, and it has also been recommended by the International Society of Renal Nutrition and Metabolism (ISRNM) for establishing a diagnosis of protein-energy wasting (8). However, its diagnostic value in the context of sarcopenia and its predictive role towards hard outcomes in patients with CKD/ESKD still remain controversial. In a previous study, our group demonstrated a weak positive correlation between MAMC and quadriceps muscle US (11). Moreover, when comparing values of abdominal skeletal muscle mass as assessed by CT with those assessed by surrogate methods, MAMC showed only a moderate degree of correlation with the reference method (24). With respect to outcomes of patients with CKD on conservative treatment, MAMC with $<90 \%$ of adequacy to the p50 value based on age and gender was used to identify patients with low muscle mass, and when combined with hand-grip strength (HGS), it was associated with survival only in the non-adjusted analysis (25). In another study in ESKD patients on HD, MAMC as a continuous variable had no association with mortality (24). Conversely, in an earlier study where MAMC was identified as percentage of agreement and analyzed as a continuous variable, this parameter independently predicted mortality (26). In the present study, US muscle parameters, but not anthropometric surrogate measures of muscle mass, namely MAMC and MAMA, were different between deceased and alive patients. In addition, when we investigated the performance of US-derived and anthropometry-derived measures of muscle size in predicting mortality with ROC analysis, the AUC of all cut-off values (p10, p25 and p50), were significant for US measurements but not for the anthropometric parameters, although the association of indexed MAMC with outcomes was significant in adjusted Cox regression 
analysis. This suggests that US-derived muscle parameters, especially distal QVIT, might be more accurate in detecting low muscle mass compared with anthropometry.

The reliability of quadriceps muscle US has been reported in critically ill patients with acute kidney injury (AKI), with excellent intraclass correlation coefficient (ICC) for inter- and intraoperator comparisons (10). In the same clinical setting, the methods used in the present study have been recently validated against CT (27). In addition, available data suggest that US is scarcely affected by fluid overload and rapid fluid shifts, such as those typical of ESKD patients on HD and patients with AKI on KRT $(10,11)$. Quadriceps muscle US has also been used in patients with CKD on conservative treatment for the assessment and validation of muscle cross-sectional area (CSA) (28). More recently, quadriceps CSA cut-offs have been derived using receiver-operation characteristic $(\mathrm{ROC})$ curves based on the presence or absence of protein energy wasting (PEW) in a Malaysian population (29). The present study confirms data reporting an association between low quadriceps muscle mass and mortality in patients on hemodialysis (30), but it is the first time that US has been used to investigate such association in this clinical setting. In fact, it has been shown that lower thigh muscle CSA as assessed by $\mathrm{CT}$ was significantly associated with all-cause and cardiovascular mortality in elderly patients on HD (30), suggesting that assessment of muscle mass of lower extremities could be particularly valuable in predicting clinical outcomes of patients on chronic HD because of its strong association with functional outcomes (31).

Because of its low discriminative value, not unexpectedly, quadriceps muscle ultrasound cannot be used as sole prognostic tool to make clinical decisions at individual level in dialysis patients, as for every other nutritional marker. Nutritional status evaluation is a comprehensive assessment that includes not only body composition, but also other clinical, social and functional parameters. On this ground, we suggest that quadriceps muscle US could add important information to multidimensional predictive models in HD patients.

Our study has some limitations. Firstly, the different cut-offs studied may not be representative of the entire ESKD population, as this multicenter study was performed in a specific population. Secondly, US and anthropometric assessments were performed by 3 different operators, which could lead to examiner-related variability. In order to limit such problem, all operators received proper training before initiation of the study. In addition, we showed in one previous paper that, operators that receive training in the same protocol of muscle US have excellent intrarater correlation coefficient (ICC) (10). Based on that background, we are confident that large inter-or intra-observer variability could not be an issue in this study, such that it would change the results. Thirdly, we did not evaluate hospital readmission, what would have provided interesting additional information. However, given the number of subjects enrolled and the prospective design of our study, we preferred to study the effect of muscle wasting assessed by US or anthropometry on the most relevant among hard outcomes, namely all-cause mortality. Finally, as we did not enroll a control group of healthy subjects, the different cutoff points were chosen arbitrarily based on specific values in the study population. Therefore, 
further investigations are warranted to identify threshold values to evaluate and define low quadriceps muscle thickness in ESKD patients on HD. Nonetheless, it should be noticed that this is the first time that the association of quadriceps muscle thickness with mortality was investigated among ESKD patients and compared to anthropometry. It is also the first time that a bedside imaging method, that has already been shown to be precise and accurate, is used to study such association. Since muscle mass is the target compartment to evaluate nutritional abnormalities in patients prone to develop chronic catabolic disorders, such as ESKD, our study is aligned with the need of investigations focusing on precise methods that can be used at the bedside.

In conclusion, low values of quadriceps muscle thickness as assessed by US and normalized by height were able to independently predict mortality in a cohort of patients on maintenance $\mathrm{HD}$, and outperformed conventional anthropometry.

Conflict of interest: Authors have no conflict of interest to declare. We also declare that the results presented in this paper have not been published previously in whole or part, except in abstract format.

Author contributions: AS, conceptualization, data curation, formal analysis, original draft; JK, supervision, review and editing; TM, data curation; CC, data curation; MCG, supervision, review and editing; SB, data review and editing, supervision; MTF, review and editing; FM, review and editing; GR, Validation, review and editing; EF, supervisor, review and editing. All authors revised and approved the final version.

Funding: non external financial support was received for this study.

Data availability statement: The data underlying this article will be shared on reasonable request to the corresponding author. 


\section{REFERENCES OF CHAPTER 6}

1. Sabatino A, Regolisti G, Karupaiah T, Sahathevan S, Sadu Singh BK, Khor BH, Salhab N, Karavetian M, Cupisti A, Fiaccadori E (2017) Protein-energy wasting and nutritional supplementation in patients with end-stage renal disease on hemodialysis. Clin Nutr 36 (3):663-671. doi:10.1016/j. clnu.2016.06.007

2. Domanski M, Ciechanowski K (2012) Sarcopenia: a major challenge in elderly patients with endstage renal disease. J Aging Res 2012:754739. doi:10.1155/2012/754739

3. Ozkayar N, Altun B, Halil M, Kuyumcu ME, Arik G, Yesil Y, Yildirim T, Yilmaz R, Ariogul S, Turgan C (2014) Evaluation of sarcopenia in renal transplant recipients. Nephrourol Mon 6 (4):e20055. doi:10.5812/ numonthly.20055

4. Shiozu H, Higashijima M, Koga $\mathrm{T}$ (2015) Association of sarcopenia with swallowing problems, related to nutrition and activities of daily living of elderly individuals. J Phys Ther Sci 27 (2):393-396. doi:10.1589/jpts.27.393

5. Argiles JM, Campos N, Lopez-Pedrosa JM, Rueda R, Rodriguez-Manas L (2016) Skeletal Muscle Regulates Metabolism via Interorgan Crosstalk: Roles in Health and Disease. J Am Med Dir Assoc 17 (9):789-796. doi:10.1016/j.jamda.2016.04.019

6. Wolfe RR (2006) The underappreciated role of muscle in health and disease. Am J Clin Nutr 84 (3):475-482. doi:10.1093/ajcn/84.3.475

7. Demling RH (2009) Nutrition, anabolism, and the wound healing process: an overview. Eplasty 9:e9

8. Fouque D, Kalantar-Zadeh K, Kopple J, Cano N, Chauveau P, Cuppari L, Franch H, Guarnieri G, Ikizler TA, Kaysen G, Lindholm B, Massy Z, Mitch W, Pineda E, Stenvinkel P, Treviño-Becerra A, Trevinho-Becerra A, Wanner C (2008) A proposed nomenclature and diagnostic criteria for protein-energy wasting in acute and chronic kidney disease. Kidney Int 73 (4):391-398. doi:10.1038/sj.ki.5002585

9. Connolly B, MacBean V, Crowley C, Lunt A, Moxham J, Rafferty GF, Hart N (2015) Ultrasound for the assessment of peripheral skeletal muscle architecture in critical illness: a systematic review. Crit Care Med 43 (4):897-905. doi:10.1097/CCM.0000000000000821

10. Sabatino A, Regolisti G, Bozzoli L, Fani F, Antoniotti R, Maggiore U, Fiaccadori E (2017) Reliability of bedside ultrasound for measurement of quadriceps muscle thickness in critically ill patients with acute kidney injury. Clin Nutr 36 (6):1710-1715. doi:10.1016/j.clnu.2016.09.029

11. Sabatino A, Regolisti G, Delsante M, Di Motta T, Cantarelli C, Pioli S, Grassi G, Batini V, Gregorini M, Fiaccadori E (2019) Noninvasive evaluation of muscle mass by ultrasonography of quadriceps femoris muscle in End-Stage Renal Disease patients on hemodialysis. Clin Nutr 38 (3):1232-1239. doi:10.1016/j.clnu.2018.05.004

12. Sabatino A, Regolisti G, di Mario F, Ciuni A, Palumbo A, Peyronel F, Maggiore U, Fiaccadori E (2020) Validation by CT Scan of Quadriceps Muscle Thickness Measurement by Ultrasound in Acute Kidney Injury. J Nephrol 33 (1):109-117. doi:10.1007/s40620-019-00659-2 
13. Sabatino A, D’Alessandro C, Regolisti G, di Mario F, Guglielmi G, Bazzocchi A, Fiaccadori E (2020) Muscle mass assessment in renal disease: the role of imaging techniques. Quant Imaging Med Surg 10 (8):1672-1686. doi:10.21037/qims.2020.03.05

14. Tillquist M, Kutsogiannis DJ, Wischmeyer PE, Kummerlen C, Leung R, Stollery D, Karvellas CJ, Preiser JC, Bird N, Kozar R, Heyland DK (2014) Bedside ultrasound is a practical and reliable measurement tool for assessing quadriceps muscle layer thickness. JPEN J Parenter Enteral Nutr 38 (7):886-890. doi:10.1177/0148607113501327

15. Carrero JJ, Chmielewski M, Axelsson J, Snaedal S, Heimburger O, Barany P, Suliman ME, Lindholm B, Stenvinkel P, Qureshi AR (2008) Muscle atrophy, inflammation and clinical outcome in incident and prevalent dialysis patients. Clin Nutr 27 (4):557-564. doi:10.1016/j.clnu.2008.04.007

16. Miyamoto T, Carrero JJ, Qureshi AR, Anderstam B, Heimburger O, Barany P, Lindholm B, Stenvinkel P (2011) Circulating follistatin in patients with chronic kidney disease: implications for muscle strength, bone mineral density, inflammation, and survival. Clin J Am Soc Nephrol 6 (5):1001-1008. doi:10.2215/cjn.10511110

17. Beddhu S, Pappas LM, Ramkumar N, Samore M (2003) Effects of body size and body composition on survival in hemodialysis patients. J Am Soc Nephrol 14 (9):2366-2372. doi:10.1097/01. asn.0000083905.72794.e6

18. Noori N, Kopple JD, Kovesdy CP, Feroze U, Sim JJ, Murali SB, Luna A, Gomez M, Luna C, Bross R, Nissenson AR, Kalantar-Zadeh K (2010) Mid-arm muscle circumference and quality of life and survival in maintenance hemodialysis patients. Clin J Am Soc Nephrol 5 (12):2258-2268. doi:10.2215/cjn.02080310

19. Streja E, Molnar MZ, Kovesdy CP, Bunnapradist S, Jing J, Nissenson AR, Mucsi I, Danovitch GM, Kalantar-Zadeh K (2011) Associations of pretransplant weight and muscle mass with mortality in renal transplant recipients. Clin J Am Soc Nephrol 6 (6):1463-1473. doi:10.2215/cjn.09131010

20. Oterdoom LH, van Ree RM, de Vries AP, Gansevoort RT, Schouten JP, van Son WJ, Homan van der Heide JJ, Navis G, de Jong PE, Gans RO, Bakker SJ (2008) Urinary creatinine excretion reflecting muscle mass is a predictor of mortality and graft loss in renal transplant recipients. Transplantation 86 (3):391-398. doi:10.1097/TP.0b013e3181788aea

21. Foley RN, Wang C, Ishani A, Collins AJ, Murray AM (2007) Kidney function and sarcopenia in the United States general population: NHANES III. Am J Nephrol 27 (3):279-286. doi:10.1159/000101827

22. Giglio J, Kamimura M, Lamarca F, Rodrigues J, Santin F, Avesani C (2018) Association of Sarcopenia With Nutritional Parameters, Quality of Life, Hospitalization, and Mortality Rates of Elderly Patients on Hemodialysis. J Ren Nutr 28 (3):197-207. doi:10.1053/j.jrn.2017.12.003

23. Carrero JJ, Johansen KL, Lindholm B, Stenvinkel P, Cuppari L, Avesani CM (2016) Screening for muscle wasting and dysfunction in patients with chronic kidney disease. Kidney Int 90 (1):53-66. doi:10.1016/j.kint.2016.02.025

24. Giglio J, Kamimura M, Souza N, Bichels A, Cordeiro A, Pinho N, Avesani C (2019) Muscle Mass Assessment by Computed Tomography in Chronic Kidney Disease Patients: Agreement With Surrogate Methods. Eur J Clin Nutr 73 (1). doi:10.1038/s41430-018-0130-1 
25. Pereira R, Cordeiro A, Avesani C, Carrero J, Lindholm B, Amparo F, Amodeo C, Cuppari L, Kamimura M (2015) Sarcopenia in Chronic Kidney Disease on Conservative Therapy: Prevalence and Association With Mortality. Nephrol Dial Transplant 30 (10). doi:10.1093/ndt/gfv133

26. Araújo I, Kamimura M, Draibe S, Canziani M, Manfredi S, Avesani C, Sesso R, Cuppari L (2006) Nutritional parameters and mortality in incident hemodialysis patients. J Ren Nutr 16 (1):27-35. doi:10.1053/j.jrn.2005.10.003

27. Sabatino A RG, di Mario F, Ciuni A, Palumbo A, Peyronel F, Maggiore U, Fiaccadori E (2019) Validation by CT scan of quadriceps muscle ultrasound in acute kidney injury. J Nephrol Epub ahead of print

28. Souza VA, Oliveira D, Cupolilo EN, Miranda CS, Colugnati FAB, Mansur HN, Fernandes N, Bastos MG (2018) Rectus femoris muscle mass evaluation by ultrasound: facilitating sarcopenia diagnosis in pre-dialysis chronic kidney disease stages. Clinics (Sao Paulo, Brazil) 73:e392. doi:10.6061/clinics/2018/e392

29. Sahathevan S, Khor B, Singh B, Sabatino A, Fiaccadori E, Daud Z, Ali M, Narayanan S, Tallman D, Chinna K, Goh B, Gafor A, Ahmad G, Morad Z, Khosla P, Karupaiah T, None OBOTPSMI (2020) Association of Ultrasound-Derived Metrics of the Quadriceps Muscle with Protein Energy Wasting in Hemodialysis Patients: A Multicenter Cross-Sectional Study. Nutrients 12 (11):3597. doi:10.3390/nu12113597

30. Fukasawa H, Kaneko M, Niwa H, Matsuyama T, Yasuda H, Kumagai H, Furuya R (2017) Lower Thigh Muscle Mass Is Associated With All-Cause and Cardiovascular Mortality in Elderly Hemodialysis Patients. Eur J Clin Nutr 71 (1). doi:10.1038/ejcn.2016.186

31. Tsukasaki K, Matsui Y, Arai H, Harada A, Tomida M, Takemura M, Otsuka R, Ando F, Shimokata H (2020) Association of Muscle Strength and Gait Speed with Cross-Sectional Muscle Area Determined by Mid-Thigh Computed Tomography - A Comparison with Skeletal Muscle Mass Measured by Dual-Energy X-Ray Absorptiometry. J Frailty Aging 9 (2):82-89. doi:10.14283/jfa.2020.16 



\section{CHAPTER 7}

Sarcopenia diagnosed by quadriceps muscle thickness assessed by ultrasound and handgrip strength predicts mortality in patients on hemodialysis and outperforms bioimpedance spectroscopy 


\section{ABSTRACT}

Background and aims: Estimation of muscle mass is a pivotal component in the diagnosis of protein-energy wasting and sarcopenia - both associated with increased mortality rates in patients on hemodialysis (HD). While bioimpedance spectroscopy (BIS) is well-known for the assessment of lean tissue for the diagnosis of sarcopenia, ultrasound (US) is not yet formally accepted. The aim of this study is to compare the predictive value for outcome between the indexed thickness of the distal quadriceps vastus intermedius (QVIT) measured by US with that from the lean tissue index (LTI) measured by BIS, combined with handgrip strength (HGS).

Methods: The cutoff values for low HGS were $<27 \mathrm{~kg}$ for males and $<16 \mathrm{~kg}$ for females, for low LTI the cut off was obtained from an age-matched healthy control group with $\mathrm{LTI}<10^{\text {th }}$ percentile, and for the low QVIT, the cutoff was $<3.44 \mathrm{~mm} / \mathrm{m}^{2}$ for males and $<3.52 \mathrm{~mm} / \mathrm{m}^{2}$ for females.

Results: In this secondary analysis of a multicenter study, US and BIS were performed in 99 patients, and HGS in 64 patients, on chronic HD. The correlation between LTI and QVIT was moderate $(r=0.37 ; p<0.001)$. After a median follow-up period of 28 months (interquartile range 19-41 months), 38 patients died. When assessing the association with mortality, the crude analysis showed that low LTI had no association with mortality (HR 1.16 [95\% Cl 0.60-2.25], while low QVIT (HR 2.18 [95\% Cl 1.12-4.27]) and low HGS (HR 9.08 [95\% confidence interval (CI) 2.1238.8]) showed to increase the mortality risk. In the fully adjusted model (adjusted for age, serum albumin, COPD, heart failure, cerebro-cardiovascular diseases), only the combination of low HGS and low QVIT was significantly associated to higher mortality risk [HR 3.21 [95\% Cl 1.37-7.53].

Conclusion: In the present study, quadriceps muscle US outperformed BIS in predicting mortality risk. US may be a useful technique in the assessment of sarcopenia and PEW in patients on HD. The combination of low HGS and QVIT index is an independent predictor of outcome in this patient group. 


\section{INTRODUCTION}

Protein energy wasting (PEW) is common in patients with advanced chronic kidney disease (CKD) with an increasing prevalence in more advanced stages (1). For the diagnosis of PEW, one of the criteria is low muscle mass (2). Moreover, sarcopenia, another prevalent nutritional disturbance observed in CKD patients (3), also uses the criteria of low muscle mass, in addition to low muscle strength, for the diagnosis of sarcopenia (4). Both PEW as well as sarcopenia are related to frailty, reduced health-related quality of life and an increased risk of mortality in patients with advanced CKD $(3,5-7)$.

For the estimation of muscle mass, various methods are available. As muscle mass is a key component of lean body mass (LBM) and fat free mass (FFM) (8), techniques such as bioelectrical impedance analysis (BIA) and dual energy X-ray absorptiometry (DEXA) are frequently used for the assessment of low muscle mass. FFM and LBM by single-frequency BIA are conventionally estimated from population-derived prediction equations including reactance and/or resistance (9), or, in case of bioimpedance spectroscopy (BIS), by the estimation of extracellular and intracellular volumes (10). While DEXA measures appendicular LBM, that mostly represents the appendicular skeletal muscle. However, both BIA/BIS and DEXA can be influenced by fluid overload in patients with advanced CKD, that because the excess extracellular water (ECW) - normally observed in this patient group, is included in the predictive equations of FFM and $\operatorname{LBM}(11,12)$. However, with the 3 compartment BIS model, the excess ECW is expressed as a separate compartment from LBM, called overhydration, next to the adipose tissue mass and lean tissue mass, thus correcting for fluid overload (13). When considering the assessment of a specific muscle or of muscular group, only computed tomography (CT) or magnetic resonance imaging (MRI), or yet US can be used. While CT and MRI are not portable, used equipment requiring high-cost maintenance and skillful personal, in addition to expose to radiation (in case of CT), US is portable, can be readily performed at bedside or in an ambulatory setting and requires much easier training skills $(14,15)$. US of the quadriceps femoris also is not affected by fluid overload (16), and showed a good correlation with CT in patients with acute kidney injury (17). Moreover, it allows direct visualization of muscle size exempting the use of predictive equations. Although muscle ultrasound was not endorsed by the revised EWGSOP2 to assess muscle mass (4), its potential due to the easyof-measurement for muscle size justifies the assessment of the performance of muscle US compared with other surrogates to predict mortality in patients on HD.

Considering the above said, the aims of the present study are firstly to compare quadriceps muscle thickness by US and lean tissue index (LTI) assessed by BIS for the prediction of mortality of patients on HD; secondly we also aim to evaluate to which extent sarcopenia, diagnosed by respectively US or BIS in combination with HGS, is related to mortality. Lastly, the correlation between quadriceps muscle thickness assessed by US and LTI evaluated by BIS was also studied. 


\section{PATIENTS \& METHODS}

\section{STUDY DESIGN AND PATIENTS}

This is a secondary analysis involving 99 MHD patients from 3 dialysis centers from northern and central Italy ( 1 in Reggio Emilia and 2 in Livorno), that participated in an ongoing observational, longitudinal and prospective study of 181 MHD patients from 6 Italian dialysis centers ( 3 in Parma, 1 in Reggio Emilia and 2 in Livorno) and that had undergone BIS evaluation at the time of the US assessment (18). The study was approved by the local Institutional Review Board (ref. $n^{\circ} 45737$, December 12th, 2015). The procedures were in agreement with the Declaration of Helsinki, and written informed consent was obtained from all participants. Patients were evaluated between January 2016 and March 2018 and followed for mortality events for a median of 28 months (interquartile range [IQR] 19-36 months). The main objective of this cohort was to assess the correlation between muscle mass by ultrasound and other nutritional parameters (18). Only adult patients ( $\geq 18$ years of age) on dialysis for at least 6 months were enrolled. Exclusion criteria were the presence of malignancy or conditions with mandatory immobilization.

\section{ULTRASOUND TECHNIQUE}

Quadriceps vastus intermedius thickness (QVIT) were measured by three experienced assessors (one for all centers in Parma and Reggio Emilia, 1 for each center from Livorno) using B-mode ultrasonography, wall tracking ultrasound system (Philips hd7xe, Logiq and General electric) and $7.5 \mathrm{MHz}$ linear array transducers. The technique used was already described in detail elsewhere $(16,18)$. In short, measurements were performed in both legs with the patient lying in a supine position with both knees extended but relaxed and toes pointing to the ceiling, during the HD session. Quadriceps vastus intermedius thickness (QVIT) was measured at the border between the lower third and upper two-thirds (QVIT Dist) between the anterior superior iliac spine (ASIS) and the upper pole of the patella $(16,19)$. The transducer was placed perpendicular to the long axis of the thigh with a large amount of gel and with no pressure to avoid compression of the muscle. The vertical diameter of the muscles was measured at the widest point, on the inner edge of the muscle fascia. Measurements were performed directly on the ultrasound machine while obtaining the images. The average of both legs was used for the analysis. For further analyses, measurements were normalized by height squared in meter. Based on data obtained from analysis performed in the whole cohort of 181 patients enrolled originally, low muscle mass by US was defined by QVIT Dist index $<3.52 \mathrm{~mm} / \mathrm{m} 2$ for females and $<3.44 \mathrm{~mm} / \mathrm{m} 2$ for males, as this cutoff (percentile 50 th of the group) was associated with the highest accuracy for predicting mortality (Chapter 6).

\section{BIOIMPEDANCE SPECTROSCOPY}

Body composition was assessed using the Body Composition Monitor (BCM, Fresenius Medical Care). Because of logistic reasons, all patients were evaluated immediately before the midweek dialysis session. Electrodes were placed on the hand of the arm without the arteriovenous 
fistula and on the same-side foot, and the device immediately and noninvasively measured whole body bioimpedance spectroscopy at 50 different frequencies between $5 \mathrm{kHz}$ and $1 \mathrm{MHz}$. The measurements were performed after patients had rested in the supine position for at least 5 min. The BCM distinguish 3 major body compartments: excess fluid mass (overhydration, free fluid), normally hydrated adipose tissue mass, and normally hydrated lean tissue mass (LTM). The lean tissue index (LTI) is the LTM normalized by height squared. The LTI was used as a surrogate of muscle mass. LTI values normalized by age and gender were used as the cut-off, patients with LTI less than $10 \%$ of the normal value were considered to have low muscle mass, and patients with LTI of $10 \%$ or greater were considered to have normal muscle mass (20-22).

\section{MUSCLE STRENGTH}

Muscle strength was assessed by hand-grip strength (HGS) with a portable digital dynamometer (KERN \& SOHN GmbH, Germany) preferably at the dominant hand. After a period to let participants arrange the instrument comfortably in their hand, they were requested to squeeze with maximum strength and minimal wrist extension (30 degrees or less). Measurements were performed before the HD session, on the arm opposite to the arteriovenous fistula (FAV), two measurements were made, after a resting period of 15 seconds between tests, and the highest measurement was recorded. The cut-off values used were: $27 \mathrm{~kg}$ for men and $16 \mathrm{~kg}$ for women (4). HGS was available for 64 patients.

\section{SARCOPENIA DIAGNOSIS}

Patients were diagnosed as having sarcopenia when both conditions, low muscle strength and low muscle mass were below the reference values (4). For the assessment of low muscle mass, both LTI assessed by BIS and QVIT Dist Index assessed by US were used.

\section{STATISTICAL ANALYSIS}

Results are expressed as mean and standard deviation for continuous variables with normal distribution, or median and range for non-parametric data, and as frequencies for categorical variables. The correlation between quadriceps muscle thickness assessed by US and other nutritional variables (in particular, LTI and HGS) was investigated using Spearman correlation coefficient. Group differences were analyzed using Student t test and Mann-Whitney's U test for normally distributed and non-normally distributed data, respectively, to assess differences between means of deceased and living patients. Kaplan-Meier survival curves were fitted to estimate the probability of mortality based on the presence of low HGS, low QVIT Dist index, low LTI, and sarcopenia diagnosed using low LTI and low QVIT Dist index. Cox-regression analysis was performed to study the risk of mortality in the presence of low HGS, low QVIT Dist index, low LTI, and sarcopenia diagnosed using both muscle parameters with mortality risk. We tested different multivariable Cox regression models with sequential adjustment for confounders identified by univariate analysis (age, albuminemia, chronic obstructive pulmonary disease, heart failure and the presence of cerebro-cardiovascular diseases). All analyses were 
computed using IBM Statistical Package for Social Sciences version 27.0 (IBM SPSS Statistics Inc. Chicago IL. USA). Statistical significance was set at $\mathrm{p}<0.05$ for all tested parameters.

\section{RESULTS}

\section{CLINICAL, DEMOGRAPHIC AND NUTRITIONAL CHARACTERISTICS OF ENROLLED PATIENTS}

In this secondary analysis, 99 patients that had undergone a BIS evaluation at the time of US were considered, of whom 64 had information regarding HGS (Table 1). The majority of patients were male, and the mean age of this cohort was $66 \pm 17$ years. In general patients were polymorbid, with only $6(6 \%)$ having no comorbidities and 19 (19\%) patients having only one comorbidity. Nutritional characteristics of the cohort, total and divided by sex, are described in Table 2. In particular, the mean value of HGS for male patients was below the cutoffs for the diagnosis of sarcopenia (4).

Table 1. Demographic and clinical characteristics of patients on hemodilaysis enrolled in the study ( $n=99)$.

\begin{tabular}{ll} 
Variables & Values \\
\hline Age (years) & $66 \pm 17$ \\
\hline Male sex (\%) & $69 / 99(70)$ \\
\hline Dialysis vintage (months) & $4.8(1.3-8.1)$ \\
\hline Dialysis modality (\%) & $60 / 99(61)$ \\
- HD & $34 / 99(34)$ \\
- HDF & $5 / 99(5)$ \\
- HFR & \\
\hline Biochemical values (Pre-dialysis) & $70 \pm 20.7$ \\
\hline BUN (mg/dl) & $9.2 \pm 2.6$ \\
\hline SCr (mg/dl) & $3.7 \pm 0.4$ \\
\hline Albumin (g/dl) & $0.6(0.2-1.1)$ \\
\hline CRP (mg/dl) & \\
\hline Chronic comorbidities (\%) & $71 / 99(72)$ \\
\hline - Hypertension & $60 / 99(61)$ \\
- Dyslipidemia & $32 / 99(32)$ \\
- Diabetes mellitus & $14 / 99(14)$ \\
- Heart failure & $46 / 99(47)$ \\
- Cardiovascular diseases & $2.6 \pm 1.7$ \\
\hline - Number of comorbidities per patient & $38 / 99(38)$ \\
\hline Mortality (\%) & $28(19-41)$ \\
Duration of follow-up(median, interquartile range) (months) & \\
\hline & \\
\hline
\end{tabular}

BUN, blood urea nitrogen; COPD, Chronic obstructive pulmonary disease; CRP, C-reactive protein; HD, hemodialysis; HDF, hemodiafiltration; HFR, hemofiltration; $\mathrm{sCr}$, serum creatinine. Data expressed as mean (standard deviation), except when otherwise specified. Data expressed as mean \pm standard deviation; median (interquartile range); or frequencies 
Table 2. Nutritional characteristic of patients on hemodialysis enrolled in the study.

\begin{tabular}{|c|c|c|c|}
\hline Variables & Total $(n=99)$ & Male $(n=69)$ & Female $(n=30)$ \\
\hline Body weight (kg) & $68.5 \pm 12.5$ & $70.9 \pm 11.6$ & $62.9 \pm 12.8$ \\
\hline Height (m) & $1.66 \pm 0.09$ & $1.70 \pm 0.06$ & $1.57 \pm 0.06$ \\
\hline BMI (kg/m2) & $24.8 \pm 4.6$ & $24.5 \pm 4.2$ & $25.4 \pm 5.4$ \\
\hline Hand-grip strength $(\mathrm{kg})^{\star}$ & $22.4 \pm 8.6$ & $24.7 \pm 8.0$ & $16 \pm 7.1$ \\
\hline \multicolumn{4}{|l|}{ Bioimpedance Spectroscopy } \\
\hline - Body cell mass (Kg) & $17.9 \pm 6$ & $18.4 \pm 6.5$ & $16.9 \pm 4.4$ \\
\hline - Lean tissue index (kg/m2) & $12.5 \pm 3.3$ & $12.1 \pm 3.3$ & $13.2 \pm 3.3$ \\
\hline - Adipose tissue mass (Kg) & $32.1 \pm 14.4$ & $33.5 \pm 14.8$ & $28.7 \pm 13.2$ \\
\hline - Fatty tissue index (kg/m2) & $11.5 \pm 5.2$ & $11.6 \pm 5.2$ & $11.5 \pm 5.4$ \\
\hline \multicolumn{4}{|c|}{ Muscle Ultrasound (average values between both legs)( $\mathrm{mm}$ ) } \\
\hline - VI Dist (mm) & $10.0 \pm 3.7$ & $10.6 \pm 4.0$ & $8.6 \pm 2.6$ \\
\hline - VI Dist index $\left(\mathrm{mm} / \mathrm{m}^{2}\right)$ & $3.6 \pm 1.3$ & $3.7 \pm 1.4$ & $3.5 \pm 1.0$ \\
\hline \multicolumn{4}{|l|}{ Sarcopenia and sarcopenia parameters } \\
\hline LTI $<10$ th & $40 / 99(40)$ & $36 / 69(52)$ & $4 / 30(13)$ \\
\hline Low VI Dist index & $52 / 99(52)$ & $36 / 69(52)$ & $16 / 30(53)$ \\
\hline Low HGS* & $39 / 64(61)$ & $31 / 47(66)$ & $8 / 17(47)$ \\
\hline Sarcopenia by LTI and HGS** & $34 / 93(34)$ & $31 / 64(48)$ & $3 / 29(10)$ \\
\hline Sarcopenia by VI Dist index and HGS & $23 / 78(30)$ & $19 / 55(53)$ & $4 / 23(17)$ \\
\hline
\end{tabular}

* Available for 64 patients (17 females and 47 males). ${ }^{* \star}$ Available for 93 patients, patients with low LTI but no information on HGS were excluded $(n=7) .{ }^{* \star *}$ Available for 78 patients, patients with low VI Dist index but no information on HGS were excluded $(n=21)$. BMI, body mass index; LTI, lean tissue index; RF, rectus femoris; VI, vastus intermedius. Data expressed as mean \pm standard deviation.

\section{CORRELATION BETWEEN QUADRICEPS MUSCLE THICKNESS AND NUTRITIONAL VARIABLES}

Table 3 reports the results on the correlation between QVIT Dist and QVIT Dist index assessed by US and LTI, as well as muscle strength by HGS. In particular, HGS correlated moderately with QVIT Dist. The correlation lost strength, but was still significant when comparing to QVIT Dist index. LTI had a low to moderate correlation with HGS and QVIT Dist, and the results were similar for the indexed muscle thickness.

Table 3. Correlation coefficients between muscle ultrasound and nutritional variables of patients on hemodilaysis $(n=99)$.

\begin{tabular}{lllllll} 
& VI Dist & $\mathbf{P}$ & VI Dist index & $\mathbf{P}$ & LTI & P \\
\hline HGS $^{*}$ & 0.51 & $<0.001$ & 0.39 & 0.001 & 0.31 & 0.014 \\
\hline LTI & 0.37 & $<0.001$ & 0.39 & $<0.001$ & - & -
\end{tabular}

HGS, Hand-grip strength; LTI, lean tissue index; VI, vastus intermedius.

*Available for 64 patients. 


\section{COMPARISON BETWEEN DECEASED AND LIVING PATIENTS}

Patients were stratified based on survival status (Table 4). The mortality prevalence was $38.4 \%$ (38/99). Eighty-four percent of deceased patients were male, and when compared to living patients, the former group was significantly older, had lower HGS and lower quadriceps muscle thickness index. The LTI, which is a surrogate of muscle mass, did not differ between groups. Among the deceased patients, the prevalence of sarcopenia by LTI was lower than that by QVIT Dist index (30\% versus 52\%). In addition, when muscle US was used to identify patients with sarcopenia, its prevalence among deceased patients was higher in comparison to living patients ( $P=0.002$ ), while no difference between both groups was observed when LTI was used.

Table 4. Main characteristic of patients on hemodialysis stratified by survival status ( $n=99)$.

\begin{tabular}{|c|c|c|c|}
\hline & $\begin{array}{l}\text { Deceased } \\
(n=38)\end{array}$ & $\begin{array}{l}\text { Alive } \\
(n=61)\end{array}$ & P-value \\
\hline Male sex (\%) & $32 / 38(84)$ & $37 / 61(61)$ & 0.013 \\
\hline Age (years) & $74.1 \pm 13.6$ & $61.6 \pm 17.1$ & $<0.001$ \\
\hline $\mathrm{BMI}(\mathrm{Kg} / \mathrm{m} 2)$ & $24.6 \pm 3.9$ & $25.0 \pm 5.0$ & 0.680 \\
\hline CRP (mg/dl) & $0.8(0.3 ; 16.5)$ & $0.3(0.2 ; 7.0)$ & 0.496 \\
\hline Albumin (g/dl) & $3.6 \pm 0.3$ & $3.8 \pm 0.4$ & 0.007 \\
\hline $\operatorname{HGS}(\mathrm{Kg})^{\star}$ & $18.9 \pm 7.2$ & $24.3 \pm 8.8$ & 0.015 \\
\hline \multicolumn{4}{|l|}{ Comorbidities and outcome } \\
\hline - Hypertension & $28 / 38(74)$ & $43 / 61(70)$ & 0.732 \\
\hline - Dyslipidemia & $23 / 38(61)$ & $37 / 61(61)$ & 0.990 \\
\hline - Diabetes & $16 / 38(42)$ & $16 / 61(26)$ & 0.100 \\
\hline- COPD & $6 / 38(16)$ & $2 / 61(3)$ & 0.026 \\
\hline - Heart failure & $10 / 38(26)$ & $4 / 61(6)$ & 0.006 \\
\hline - Cerebro-cardiovascular diseases & $28 / 38(74)$ & $18 / 61(30)$ & $<0.001$ \\
\hline \multicolumn{4}{|l|}{ Bioimpedance Spectroscopy } \\
\hline - Body cell mass (Kg) & $17.3 \pm 5.9$ & $18.3 \pm 6.0$ & 0.433 \\
\hline - Lean tissue index $(\mathrm{kg} / \mathrm{m} 2)$ & $12.0 \pm 3.2$ & $12.7 \pm 3.3$ & 0.288 \\
\hline - Fat tissue index $(\mathrm{kg} / \mathrm{m} 2)$ & $11.9 \pm 4.5$ & $11.3 \pm 5.7$ & 0.570 \\
\hline \multicolumn{4}{|c|}{ Muscle Ultrasound (average values between both legs)(cm) } \\
\hline - VI Prox index & $4.2 \pm 1.3$ & $5.3 \pm 2.1$ & 0.006 \\
\hline - VI Dist index & $3.2 \pm 1.0$ & $3.9 \pm 1.4$ & 0.007 \\
\hline \multicolumn{4}{|l|}{ Sarcopenia and sarcopenia parameters } \\
\hline $\mathrm{LTI}<10^{\text {th }}$ percentile & $15 / 38(39)$ & $25 / 61(41)$ & 0.882 \\
\hline Low VI Dist index & $25 / 38(66)$ & $27 / 61(44)$ & 0.037 \\
\hline Low HGS* & $21 / 23(91)$ & $18 / 41(44)$ & $<0.001$ \\
\hline Sarcopenia by LTI and HGS** & $10 / 33(30)$ & $24 / 60(40)$ & 0.353 \\
\hline 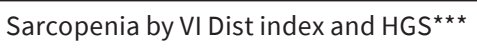 & $14 / 27(52)$ & $9 / 51(18)$ & 0.002 \\
\hline
\end{tabular}

*Available for 64 patients. * Available for 93 patients, patients with low LTI but no information on HGS were excluded $(n=7) .{ }^{\star \star \star}$ Available for 78 patients, patients with low VI Dist index but no information on HGS were excluded $(n=21)$. Data expressed as mean (standard deviation). HGS, hand-grip strength; LTI, lean tissue index; RF, rectus femoris; VI, vastus intermedius. 
In order to assess the probability of outcome in the presence of low muscle strength and low muscle mass, we fitted Kaplan-Meier survival curves using the sarcopenia parameters individually (Figure $1 \mathrm{~A}-\mathrm{C}$ ) and combined (Figure 2A and B). When parameters were analyzed individually, only HGS and QVIT Dist index were related to the cumulative survival (Figure $1 \mathrm{~A}-\mathrm{C})$. When evaluating the correlation between sarcopenia and survival, only sarcopenia assessed by QVIT Dist index and HGS was associated with the cumulative survival (Figure 2A-B). We performed Cox-regression analysis to assess the effect of such parameters on mortality risk (Table 5). When muscle strength and muscle mass were analyzed individually, only low HGS and low QVIT Dist index increased mortality risk. This association lost significance in the full adjusted analysis. On the other hand, when sarcopenia was diagnosed by QVIT Dist index and HGS, the increased mortality risk remained significant also in the full adjusted analysis.

Figure 1A-C. Kaplan-Meier survival curves of patients with end-stage kidney disease on hemodialysis divided by individual sarcopenia parameters: muscle strength assessed by handgrip (A), muscle mass assessed by QVIT Dist index (B) and lean tissue index (C)

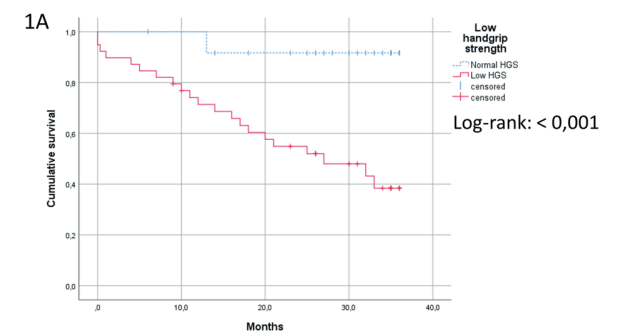

$1 \mathrm{~B}$
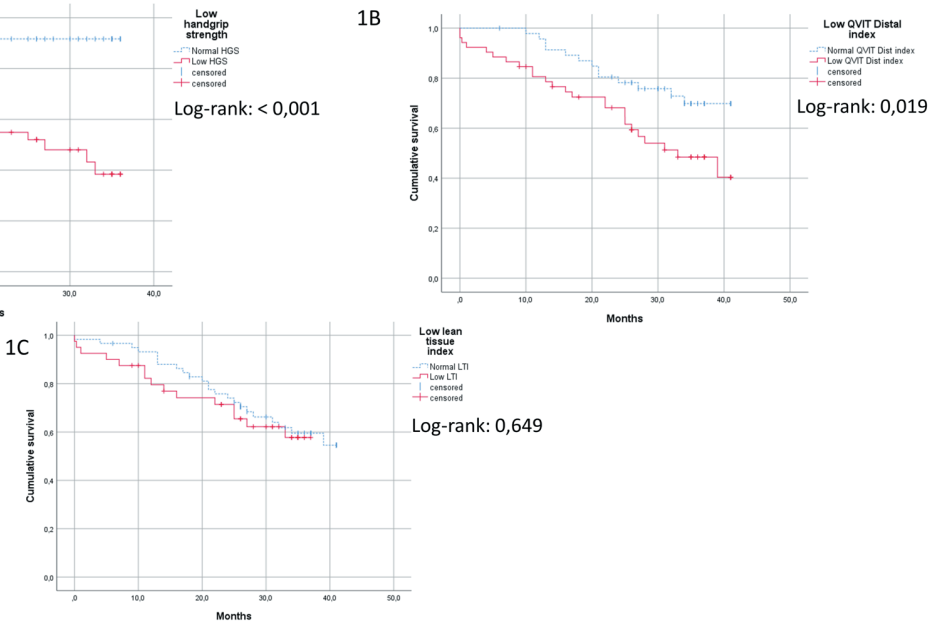

Legend. (A) Handgrip strength was assessed in 64 patients; (B) QVIT Dist index was assessed in 99 patients; (C) Lean tissue index was assessed in 99 patients. 
Figure 2A-B. Kaplan-Meier survival curves of patients with end-stage kidney disease on hemodialysis divided by sarcopenic status using low QVIT Dist index (A) and low LTI (B) to assess low muscle mass.

$2 A$

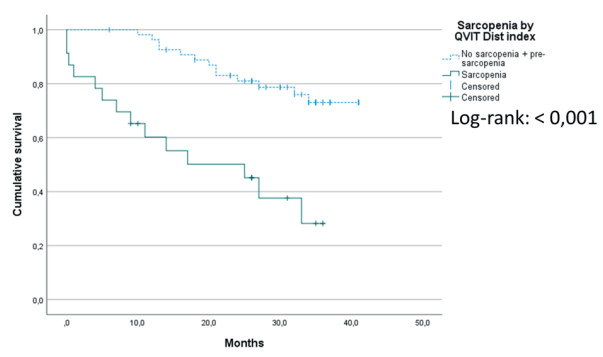

2B

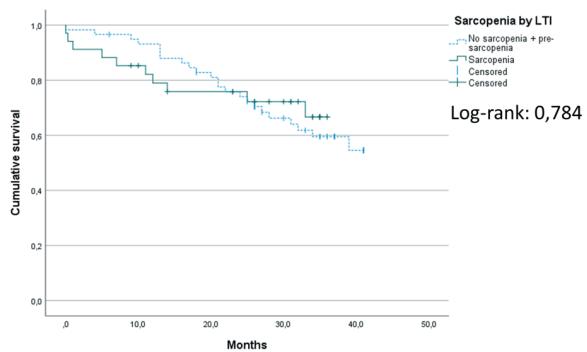

Legend. For both $A$ and $B$, sarcopenia was diagnosed when muscle mass and muscle strength were below reference values. Because handgrip strength was available for 64 patients. In the first figure (A) 78 patients were included, while 21 patients were excluded because presented low muscle mass by US but no information regarding muscle strength was present. In the second figure (B), 93 patients were included and 7 patients were excluded because presented low muscle mass by LTI but no information regarding muscle strength was available.

Table 5. Cox-regression analysis for the association of all-cause mortality with muscle parameters evaluated individually (HGS, QVIT Dist index and LTI) or combined (sarcopenia diagnosed by low LTI + HGS or low QVIT Dist index + HGS) in patients on hemodialysis ( $\mathrm{n}=64$ for HGS; $\mathrm{n}=99$ for QVIT Dist index and LTI; $n=78$ for sarcopenia by US and HGS; and $n=93$ for sarcopenia by LTI and HGS).

All-cause mortality

\begin{tabular}{|c|c|c|c|c|c|c|}
\hline \multicolumn{7}{|c|}{ HR (95\% Confidence Interval, P) } \\
\hline Model & Variables & Low LTI & Low HGS & $\begin{array}{l}\text { Low VI Dist } \\
\text { index }\end{array}$ & $\begin{array}{l}\text { Sarcopenia } \\
\text { by low VI } \\
\text { Dist Index } \\
\text { and HGS }\end{array}$ & $\begin{array}{l}\text { Sarcopenia } \\
\text { by low LTI } \\
\text { and HGS }\end{array}$ \\
\hline 1 & Non adjusted & $\begin{array}{l}1.16(0.60- \\
2.25,0.651)\end{array}$ & $\begin{array}{l}9.08(2.12- \\
38.8,0.003)\end{array}$ & $\begin{array}{l}2.18(1.12- \\
4.27,0.023)\end{array}$ & $\begin{array}{l}4.46(2.08- \\
9.56,<0.001)\end{array}$ & $\begin{array}{l}0.90(0.43- \\
1.90,0.784)\end{array}$ \\
\hline 2 & $1+$ Age + albumin & $\begin{array}{l}1.62(0.81- \\
3.23,0.171)\end{array}$ & $\begin{array}{l}5.25(1.07- \\
25.81,0.041)\end{array}$ & $\begin{array}{l}1.60(0.79- \\
3.23,0.191)\end{array}$ & $\begin{array}{l}2.87(1.25- \\
6.57,0.013)\end{array}$ & $\begin{array}{l}1.38(0.62- \\
3.04,0.429)\end{array}$ \\
\hline 3 & Full adjusted* & $\begin{array}{l}1.76(0.87- \\
3.59,0.118)\end{array}$ & $\begin{array}{l}3.55(0.69- \\
18.28,0.130)\end{array}$ & $\begin{array}{l}1.76(0.86- \\
3.58,0.122)\end{array}$ & $\begin{array}{l}3.21(1.37- \\
7.53,0.007\end{array}$ & $\begin{array}{l}1.49(0.65- \\
3.41,0.347)\end{array}$ \\
\hline
\end{tabular}

*Adjusted for age, serum albumin, COPD, heart failure, cerebro-cardiovascular diseases. HGS, hand-grip strength; LTI, lean tissue index; VI, vastus intermedius; US, ultrasound.

\section{DISCUSSION}

In the present study, we found that a low QVIT Dist index, measured by US, was significantly related to mortality in unadjusted analysis whereas low LTI, measured by BIS, was not. The combination of low HGS and low QVIT Dist index, as a potential marker for sarcopenia, was also 
related to outcome in unadjusted and full adjusted analysis, in contrast to the combination of low LTI and HGS. The results of our study are in disagreement with various other larger studies, who found a low LTI to be a predictor of adverse outcome (13, 23-25). However, in another study, low LTI was not significantly related to mortality as a single risk factor, but enhanced the risk of adverse outcomes associated with other predictors of outcome, such as inflammation or fluid overload (26).

There is a conceptual difference between US, which measures muscular dimensions, albeit at a regional level, and BIS, which estimates LTI based on various theoretical assumptions in addition to using prediction equations not always validated to dialyzed patients, resulting in moderate, but significant correlations between LTI and QVIT Dist index. Still, fluid overload assessed by BIS is consistently related to outcome (25), whereas at a population level, a consistent decline in LTI were observed following the start of HD (27).

The reason that LTI in this study was not independently related to outcome, in contrast to most, but not all (26) studies is unclear. Firstly, the sample size of our study was relatively small whereas the percentage of females with low LTI levels was low. Secondly, previous studies showed that low LTI was especially related to increased mortality in combination with other risk factors, such as inflammation, fluid overload, or a reduced fat mass $(24,26)$. In addition, our measurements of BIS were performed before the dialysis session, which may increase the influence of fluid overload over the LTI (28). Although due to its relatively low power our study cannot negate a potential relation between LTI and outcome, the predictive value of low QVIT Dist index by muscle ultrasound was consistent. This suggests that US of the quadriceps muscle, might be a more powerful tool in predicting outcome, also in combination with a low HGS.

Muscle US is relatively easy to learn, fast to perform and can also be measured by a portable device at the bedside of patients. In this regard, it's reliability (intra-operator and interoperator) and validity (against CT) have been reported in critically ill patients with AKI $(16,17)$. In addition, previous studies showed that, conversely to 2 compartment BIA/BIS models (11, 12), muscle US was not influenced by hydration status when measurements were performed before and after the dialysis session $(16,18)$. A recent study showed that ultrasound of the quadriceps muscle had a larger accuracy in predicting PEW in patients on HD as compared to BIS (14), but both techniques have not been directly compared in their predictive value for mortality in this population. A drawback of the method so far has been the absence of cutoff values. However, in our most recent study, we used the percentile distribution of indexed quadriceps muscle thickness of 181 patients in HD divided by sex to derive possible reference values based on their accuracy in predicting mortality using ROC analysis (data under review). In that study, not only QVIT Dist index at the 50th percentiles of the patient population had the highest accuracy in predicting mortality in the ROC analysis, but also, it had the highest mortality risk in the full-adjusted Cox-regression analysis (HR 2.16, $1.27-3.69, \mathrm{P}=0.005$ ) in comparison to anthropometry and other US parameters. 
The evaluation of muscle strength is essential for the diagnosis of sarcopenia (4). The screening of patients for low muscle strength, has an important advantage in comparison to evaluating muscle mass, since it can be easily assessed in hospitals, outpatient settings, and community centers, by using a portable handheld dynamometer to measure grip strength. In addition, studies in the elderly have shown that low muscle strength is better than low muscle mass in predicting worse outcomes (29). Moreover, the rate of decrease in muscle strength is faster than the rate of muscle loss, and that both are independent from the loss or gain of body weight (30). It has been suggested that CKD patients manifest a phenotype of accelerated aging (31), so the same relationship could be expected in patients with CKD/ESKD. In the present study, HGS had a positive correlation with QVIT Dist and LTI. This is in agreement with earlier studies investigating the correlation between body composition and values of physical function in hemodialysis patients $(32,33)$. A positive correlation between muscle strength and muscle mass was expected because the former is partly dependent on lean tissue (11). In addition, we found that HGS was a stronger predictor of mortality than muscle mass assessed both by BIS and US. That finding is in agreement with other longitudinal studies with ESKD patients on $\operatorname{HD}(5,34,35)$. However, only when a quantitative assessment of muscle mass was performed and sarcopenia was diagnosed, this relationship remained significant also in the full adjusted analysis. Suggesting that both parameters complete each other and are necessary for an individualized nutritional and physical intervention.

Our study has some important limitations that should be addressed. Firstly, it is a secondary analysis of a bigger study, which results in lower power to evaluate hard end-points such as mortality. Secondly, a validation of the reference values applied for muscle US should be performed in different populations, as they were derived from a specific group of ESKD patients on chronic hemodialysis. Thirdly, for logistic reasons, BIS was performed before the HD session, which might have influenced LTI values. Lastly, HGS was not available for all patients, leading to patients with reduced muscle mass but unavailable muscle strength, being removed from the analysis because it was not possible to determine the presence or absence of sarcopenia. However, this is the first time that the mortality predictability of muscle ultrasound has been compared to BIS, a well-established parameter used to assess muscle status in ESKD patients on chronic HD. In addition, our study has important potential implications for the clinical practice, adding a portable imaging tool, therefore allowing for the measurement of regional muscle, to the diagnosis of sarcopenia.

In conclusion, in the present study, quadriceps muscle US outperformed BIS in mortality prediction. Quadriceps muscle US may be a useful technique in the assessment of sarcopenia and PEW in patients on HD. The combination of low HGS and low QVIT Dist index is an independent predictor of mortality in this population. 


\section{REFERENCES OF CHAPTER 7}

1. Kovesdy CP, Kopple JD, Kalantar-Zadeh K. Management of protein-energy wasting in non-dialysis-dependent chronic kidney disease: reconciling low protein intake with nutritional therapy. Am J Clin Nutr. 2013;97(6):1163-77.

2. Fouque D, Kalantar-Zadeh K, Kopple J, Cano N, Chauveau P, Cuppari L, et al. A proposed nomenclature and diagnostic criteria for protein-energy wasting in acute and chronic kidney disease. Kidney Int. 2008;73(4):391-8.

3. Sabatino A, Cuppari L, Stenvinkel P, Lindholm B, Avesani C. Sarcopenia in chronic kidney disease: what have we learned so far? J Nephrol. 2020.

4. Cruz-Jentoft AJ, Bahat G, Bauer J, Boirie Y, Bruyere O, Cederholm T, et al. Sarcopenia: revised European consensus on definition and diagnosis. Age Ageing. 2019;48(1):16-31.

5. Giglio J, Kamimura M, Lamarca F, Rodrigues J, Santin F, Avesani C. Association of Sarcopenia With Nutritional Parameters, Quality of Life, Hospitalization, and Mortality Rates of Elderly Patients on Hemodialysis. J Ren Nutr. 2018;28(3):197-207.

6. Pereira R, Cordeiro A, Avesani C, Carrero J, Lindholm B, Amparo F, et al. Sarcopenia in Chronic Kidney Disease on Conservative Therapy: Prevalence and Association With Mortality. Nephrol Dial Transplant. 2015;30(10).

7. Kovesdy CP, Kalantar-Zadeh $\mathrm{K}$. Why is protein-energy wasting associated with mortality in chronic kidney disease? Semin Nephrol. 2009;29(1):3-14.

8. Prado C, Heymsfield S. Lean tissue imaging: a new era for nutritional assessment and intervention. JPEN J Parenter Enteral Nutr. 2014;38(8):940-53.

9. Beaudart $\mathrm{C}$, Bruyère $\mathrm{O}$, Geerinck $\mathrm{A}$, Hajaoui $\mathrm{M}$, Scafoglieri $\mathrm{A}$, Perkisas $\mathrm{S}$, et al. Equation models developed with bioelectric impedance analysis tools to assess muscle mass: A systematic review. Clinical nutrition ESPEN. 2020;35:47-62.

10. Matthie J. Bioimpedance measurements of human body composition: critical analysis and outlook. Expert review of medical devices. 2008;5(2):239-61.

11. Konings C, Kooman J, Schonck M, van Kreel B, Heidendal G, Cheriex E, et al. Influence of fluid status on techniques used to assess body composition in peritoneal dialysis patients. Perit Dial Int. 2003;23(2):184-90.

12. Lukaski $\mathrm{H}$. Evolution of bioimpedance: a circuitous journey from estimation of physiological function to assessment of body composition and a return to clinical research. Eur J Clin Nutr. 2013;67 Suppl 1:S2-S9.

13. Broers N, Canaud B, Dekker M, van der Sande F, Stuard S, Wabel P, et al. Three compartment bioimpedance spectroscopy in the nutritional assessment and the outcome of patients with advanced or end stage kidney disease: What have we learned so far? Hemodial Int. 2020;24(2):148-61. 
14. Sahathevan S, Khor B, Singh B, Sabatino A, Fiaccadori E, Daud Z, et al. Association of Ultrasound-Derived Metrics of the Quadriceps Muscle with Protein Energy Wasting in Hemodialysis Patients: A Multicenter Cross-Sectional Study. Nutrients. 2020;12(11):3597.

15. Sabatino A, D’Alessandro C, Regolisti G, di Mario F, Guglielmi G, Bazzocchi A, et al. Muscle mass assessment in renal disease: the role of imaging techniques. Quant Imaging Med Surg. 2020;10(8):1672-86.

16. Sabatino A, Regolisti G, Bozzoli L, Fani F, Antoniotti R, Maggiore U, et al. Reliability of bedside ultrasound for measurement of quadriceps muscle thickness in critically ill patients with acute kidney injury. Clin Nutr. 2017;36(6):1710-5.

17. Sabatino A, Regolisti G, di Mario F, Ciuni A, Palumbo A, Peyronel F, et al. Validation by CT Scan of Quadriceps Muscle Thickness Measurement by Ultrasound in Acute Kidney Injury. J Nephrol. 2020;33(1):109-17.

18. Sabatino A, Regolisti G, Delsante M, Di Motta T, Cantarelli C, Pioli S, et al. Noninvasive evaluation of muscle mass by ultrasonography of quadriceps femoris muscle in End-Stage Renal Disease patients on hemodialysis. Clin Nutr. 2019;38(3):1232-9.

19. Tillquist M, Kutsogiannis DJ, Wischmeyer PE, Kummerlen C, Leung R, Stollery D, et al. Bedside ultrasound is a practical and reliable measurement tool for assessing quadriceps muscle layer thickness. JPEN J Parenter Enteral Nutr. 2014;38(7):886-90.

20. Wieskotten S, Heinke S, Wabel P, Moissl U, Becker J, Pirlich M, et al. Bioimpedance-based identification of malnutrition using fuzzy logic. Physiological measurement. 2008;29(5):639-54.

21. Chamney P, Wabel P, Moissl U, Müller M, Bosy-Westphal $A$, Korth $O$, et al. A whole-body model to distinguish excess fluid from the hydration of major body tissues. Am J Clin Nutr. 2007;85(1):80-9.

22. van Biesen W, Claes K, Covic A, Fan S, Lichodziejewska-Niemierko M, Schoder V, et al. A multicentric, international matched pair analysis of body composition in peritoneal dialysis versus haemodialysis patients. Nephrol Dial Transplant. 2013;28(10):2620-8.

23. Rosenberger J, Kissova V, Majernikova M, Straussova Z, Boldizsar J. Body composition monitor assessing malnutrition in the hemodialysis population independently predicts mortality. J Ren Nutr. 2014;24(3):172-6.

24. Marcelli D, Usvyat L, Kotanko P, Bayh I, Canaud B, Etter M, et al. Body composition and survival in dialysis patients: results from an international cohort study. Clin J Am Soc Nephrol. 2015;10(7):1192-200.

25. Hwang S, Lee J, Lee S, Kim J, Kim M, Song J. Risk of overhydration and low lean tissue index as measured using a body composition monitor in patients on hemodialysis: a systemic review and meta-analysis. Ren Fail. 2018;40(1):51-9.

26. Dekker M, Konings C, Canaud B, van der Sande F, Stuard S, Raimann J, et al. Interactions Between Malnutrition, Inflammation, and Fluid Overload and Their Associations With Survival in Prevalent Hemodialysis Patients. J Ren Nutr. 2018;28(6):435-44.

27. Marcelli D, Brand K, Ponce P, Milkowski A, Marelli C, Ok E, et al. Longitudinal Changes in Body Composition in Patients After Initiation of Hemodialysis Therapy: Results From an International Cohort. J Ren Nutr. 2016;26(2):72-80. 
28. El-Kateb S, Davenport A. Changes in Intracellular Water Following Hemodialysis Treatment Lead to Changes in Estimates of Lean Tissue Using Bioimpedance Spectroscopy. Nutr Clin Pract. 2016;31(3).

29. Leong D, Teo K, Rangarajan S, Lopez-Jaramillo P, Avezum A, Orlandini A, et al. Prognostic value of grip strength: findings from the Prospective Urban Rural Epidemiology (PURE) study. Lancet (London, England). 2015;386(9990):266-73.

30. Delmonico M, Harris T, Visser M, Park S, Conroy M, Velasquez-Mieyer P, et al. Longitudinal Study of Muscle Strength, Quality, and Adipose Tissue Infiltration. Am J Clin Nutr. 2009;90(6).

31. Kooman J, Kotanko P, Schols A, Shiels P, Stenvinkel P. Chronic kidney disease and premature ageing. Nat Rev Nephrol. 2014;10(12):732-42.

32. Gadaen R, Kooman J, Cornelis T, van der Sande F, Winkens B, Broers N. The Effects of Chronic Dialysis on Physical Status, Quality of Life, and Arterial Stiffness: A Longitudinal Study in Prevalent Dialysis Patients. Nephron. 2021;145(1):44-54.

33. Martinson M, Ikizler TA, Morrell G, Wei G, Almeida N, Marcus RL, et al. Associations of body size and body composition with functional ability and quality of life in hemodialysis patients. Clin J Am Soc Nephrol. 2014;9(6):1082-90.

34. Isoyama N, Qureshi A, Avesani C, Lindholm B, Bàràny P, Heimbürger $O$, et al. Comparative Associations of Muscle Mass and Muscle Strength With Mortality in Dialysis Patients. Clin J Am Soc Nephrol. 2014;9(10):1720-8.

35. Kittiskulnam P, Chertow G, Carrero J, Delgado C, Kaysen G, Johansen K. Sarcopenia and Its Individual Criteria Are Associated, in Part, With Mortality Among Patients on Hemodialysis. Kidney Int. 2017;92(1):238-47. 



\section{CHAPTER 8}

General Discussion 
The aim of this thesis was to study the applicability, validity and prognostic capacity of quadriceps muscle ultrasound (US) in patients with acute kidney injury (AKI) and patients on chronic hemodialysis (HD) treatment, in order to develop and promote a new bedside tool to be used for the nutritional assessment of patients in these clinical contexts. To this purpose, we developed a protocol that studied all important steps involved in this process. Firstly, we studied the inter and intra-rater reliability of the US methodology to assess its reproducibility between assessors and within the same assessor. In addition, we investigated whether kidney replacement therapy (KRT) and fluid overload influenced measurements. Secondly, the methodology was validated against computed tomography (CT), a gold standard technique, to test the accuracy and precision of muscle US. Thirdly, the applicability and relevance of muscle US was studied in different situations. In patients on chronic hemodialysis (HD) quadriceps muscle of patients was compared with controls and also between malnourished and wellnourished patients to assess whether muscle US was a sensitive tool to detect low muscle mass. We also investigated its correlation with other nutritional parameters. In addition, by applying it in critically ill patients with acute kidney injury (AKI) twice during hospital stay, we studied whether muscle US was sensitive enough to detect muscle loss in short periods of time. Finally, its prognostic capacity regarding mortality risk was also investigated in two studies and compared it with other tools for the assessment of body composition, anthropometrics and bioimpedance spectroscopy (BIS).

\section{Reliability of bedside ultrasound for measurement of quadriceps muscle thickness in critically ill patients with acute kidney injury (Chapter 2)}

In the first study of this thesis we aimed to test inter- and intrarater reliability of the ultrasound technique to assess quadriceps muscle thickness, in particular rectus femoris (RF) and vastus intermedius (VI), in critically ill patients with AKI using the intraclass correlation coefficient (ICC). Before our publication, only one other study calculated the ICC to evaluate the reliability of quadriceps muscle thickness assessed by the same US methodology; however, only healthy subjects were considered and fewer measurements were performed (1). Our data confirms the excellent reliability of previous data in a much more complex population.

Because of the amount of measurements obtained for each investigator, we found that the mean difference between investigators was statistically significant; however, this difference was approximately $0.4 \mathrm{~mm}$, which is not clinically relevant. In addition, the ICC obtained in the interrater reliability analysis was high (0.92), demonstrating that this is a reliable technique to be applied by different operators that received the same training. Furthermore, there was no significant difference within each investigators' measurements. Possible small imprecisions caused by different operators assessing quadriceps muscle thickness could be easily overcome in the clinical practice by standardizing a protocol in which the same person is responsible for successive measures in the same patient.

The use of ultrasound to assess muscle mass and quality is increasing and have been validated in different populations (1-6). To our knowledge, we were the first to assess the reliability of this 
methodology in critically ill patients with AKI and to assess whether measurements of RF and VI thickness obtained by ultrasound are consistent before and after dialysis. We were also able to demonstrate that important fluid shifts caused by dialysis do not interfere with US measurements.

\section{Validation by CT scan of quadriceps muscle thickness measurement by ultrasound in acute kidney injury (Chapter 3 )}

This section of the thesis shows how US compares to CT scan for the evaluation of RF and VI thickness in critically ill patients with AKI. US bias was found to be negligible, and its precision was close to that of CT scan. Our data is in accordance to other studies that used CT for the validation of US for the assessment of muscle dimensions in different clinical settings $(3,4)$. Similarly, studies comparing muscle mass assessed by US with MRI, another gold standard technique, found no difference between both methods, with very high correlation coefficients and agreement in different populations (7-9). More recently, quadriceps muscle US has been validated against CT also in patients with chronic kidney disease (CKD) (10). To our knowledge, this is the first study that evaluated the validity of RF and VI thickness assessed by US in a cohort of critically ill patients with AKI. Earlier studies on critically ill patients compared US with muscle biopsy (11) and muscle strength (6). In both studies, muscle ultrasound was able to detect muscle loss (11), and to predict muscle strength and function at discharge (6).

\section{Noninvasive evaluation of muscle mass by ultrasonography of quadriceps femoris muscle} in End-Stage Renal Disease patients on hemodialysis (Chapter 4)

In the third part of this thesis (chapter 4) US was applied in ESKD patients on chronic HD during the dialysis session without any disturbances from fluid overload and rapid fluid shifts, confirming evidence from the reliability study (12). The main finding of this study is that patients on chronic HD have lower quadriceps muscle thickness in comparison to healthy controls. This finding is in accordance to recent data that investigated quadriceps muscle of patients on HD using MRI (13). By stratifying patients using different indexes of nutritional status, such as BMI and MIS, patients with worse nutritional status by those parameters had also lower quadriceps muscle thickness. In addition, low to moderate statistically significant correlations between muscle thickness measured by US and other nutritional variable (MIS, arm muscle area, albumin and BMI) was found, with the highest values being found for the MIS tool ( $r=-0.37$ to $-0.47, P<0.001$ for all sites of measurement). Considering that MIS is a composite of different nutritional variables, including albumin and BMI, the higher correlation with this index in comparison to the other indexes studied was not unexpected. This was the first time that quadriceps muscle US was applied in ESKD patients on chronic HD to assess their level of muscularity in comparison to controls and to confront this new tool to other frequently used and recognized nutritional indexes, providing important information toward the standardization of US as a reference bedside tool for the assessment of quadriceps muscle. 
Ultrasound for non-invasive assessment and monitoring of quadriceps muscle thickness in critically ill patients with Acute Kidney Injury (Chapter 5)

Since the combination of bedrest, AKI and critical illness are recognized catabolic factors, the sensitivity of quadriceps muscle US in detecting muscle loss in short periods of time was also tested. Interestingly, at baseline, patients admitted to the ICU had similar muscle thickness of healthy controls, however, after 5 days of hospitalization the amount of muscle thickness reduction was compatible to other available findings in which RF thickness and VI thickness after 5 days of ICU stay were reduced on average $-16.6 \%$ and $-18.8 \%$ respectively (6), and, in comparison to healthy controls, patients had lower muscle thickness.

We were also able to demonstrate, even considering the limits inherent to our small sample, that there is a direct relationship between the severity of muscle loss and prolonged hospital stay. In fact, in a similar clinical setting (surgical patients in the ICU), sarcopenia quantified by US of the RF was able to predict adverse outcomes (14). In addition, muscle loss during ICU stay is a major contributor to functional disability (15).

The assessment of muscle mass is still a challenge in the ICU setting. Biochemical parameters such as serum albumin, and currently used bedside tools that are successfully applied in the outpatient setting, such as anthropometry and bioelectrical impedance analysis (BIA) or spectroscopy (BIS), have important intrinsic limitations, due to inflammation and fluid imbalance that are typical of critically ill patients with AKI. In this regard, despite the current lack of reference values to identify low muscularity at hospital admission, quadriceps muscle US could become a useful tool to monitor muscle loss during hospitalization, and also to assess the success of physical rehabilitation.

\section{Quadriceps muscle thickness assessed by ultrasound outperforms conventional anthropometry in the evaluation of mortality risk in hemodialysis patients (Chapter 6)}

In chapter 6 , RF and VI thickness indexed by height ${ }^{2}$, were independently associated with mortality in patients on chronic HD. Taking into account that there are no available reference values derived from healthy populations, the predictive value of different cutoffs obtained from our sample distribution (p10, p25, and p50) was investigated, and the results obtained by US were compared with anthropometric measurements routinely applied in the outpatient setting and also recommended by the International Society of Renal Nutrition and Metabolism (ISRNM) for the diagnosis of PEW, namely mid arm muscle circumference (MAMC) and mid-arm muscle area (MAMA).

Muscle loss is very common in ESKD patients on chronic HD (16-18), as it is a consequence of metabolic acidosis, chronic inflammation, and anorexia related to the kidney disease per se, as well as of renal replacement therapy and comorbidities associated with increased protein catabolism and reduced protein anabolism (19). Previous available literature investigating the role of low muscularity in mortality risk, show that low muscle mass as assessed by body composition or surrogate methods increase the risk of worse outcomes (20-26). However, just 
like in the acute care setting, the bedside assessment of skeletal muscle is challenging, while gold standard techniques such as DEXA, CT and MRI are not feasible to be used on a routine basis to monitor only body composition. This was the first time that muscle US was studied in this context.

In comparison to MAMC and MAMA, US had better discriminative value, with significant AUC for all studied cut-off values but not for the anthropometric parameters. In the Cox regression analysis, indexed distal VI thickness was able to predict mortality in non-adjusted and adjusted models, while indexed MAMC was associated with mortality only in the fully adjusted analysis, suggesting that US-derived muscle parameters, especially distal QVIT, might be more accurate in detecting low muscle mass compared with anthropometry.

\section{Sarcopenia diagnosed by quadriceps muscle thickness assessed by ultrasound and hand-grip strength predicts mortality in patients on hemodialysis and outperforms bioimpedance spectroscopy (Chapter 7 )}

Finally, the value of quadriceps muscle US in diagnosing sarcopenia was investigated in comparison to BIS. In chapter 7 sarcopenia was diagnosed by identifying patients with low muscle mass and low muscle strength. Muscle strength was measured by handgrip strength (HGS), while muscle mass was measured by two different methods, BIS and quadriceps muscle US. When applying BIS, the lean tissue index (LTI) was used as a surrogate of muscle mass and values below the 10th percentile of the normal value by age and gender were considered positive for low muscle mass. When using quadriceps muscle US, we applied the percentile 50th of indexed distal VI thickness studied in the previous chapter to diagnose patients with low muscle mass, as this cutoff (percentile 50th of the group) was associated with the highest accuracy for predicting mortality.

The present chapter shows that low muscle mass by quadriceps muscle US was significantly related to mortality, while low LTI was not. In addition, sarcopenia diagnosed by HGS and US was independently correlated to outcome, while sarcopenia diagnosed by LTI and HGS was not. Our findings are a little controversial, since many larger studies found a low LTI to be a predictor of adverse outcome (27-30). That might be because of the relative small sample size of our study and also because of the low percentage of females with low LTI. In addition, previous studies confirmed that low LTI has a more powerful effect on mortality when combined with other risk factors, such as inflammation, fluid overload, or low fat mass $(28,31)$. Although due to its relatively low power our study cannot negate a potential relation between LTI and outcome, the predictive value of low indexed VI thickness by muscle US was consistent. This suggests that US of the quadriceps muscle, might be a more powerful tool in predicting outcome, also in combination with a low HGS.

The findings of this chapter reinforce the need to screen all patients on hemodialysis for muscle wasting and reduced function. Considering that low muscle strength worsens the risk 
of mortality already high in the presence of muscle wasting, both features should be assessed in combination to allow for an individualized interventional approach.

\section{STRENGTHS OF THIS THESIS}

Taken all studies together, the present thesis has many strengths. Firstly, we structured our protocol in a logical order to allow for the development of quadriceps muscle US as a bedside tool for the assessment of muscle loss in the renal setting. To achieve that objective, we firstly studied the reliability of the method in different situations, and compared measurements performed by two different operators to study its reproducibility with great results. We were also able to validate this new methodology by comparing it to $\mathrm{CT}$, a gold standard technique for the assessment of body composition. Thirdly, we performed a cross-sectional study to evaluate how US would compare to other nutritional tools and if it was able to identify patients with worse nutritional status. Following the cross-sectional study, a number of prospective studies were performed to assess US's sensitivity to be used as a monitoring tool, and its relation to mortality risk. It should be noticed that this is the first time that a bedside imaging method is used to study the association of quadriceps muscle thickness with mortality among patients on chronic HD and compared it to other bedside tools (anthropometry and BIS). Since muscle mass is the target compartment to evaluate nutritional abnormalities in patients with ESKD on HD our study has important potential implications for the clinical practice, adding a portable imaging tool for the assessment of muscle loss.

\section{LIMITATIONS OF THIS THESIS}

Some limitations should also be acknowledged. Firstly, in chapters 6 and 7 the reference values used derived from the studied population, which might not be representative of the entire HD population. In addition, the European working group for sarcopenia in older people (EWGSOP) recommends the use of normative data derived from healthy young adults whenever possible for the determination of reference values for the diagnosis of sarcopenia (32). However, we were not able to include as many healthy subjects from different ages to derive normative values to be used as cut-off, using data derived from the studied population instead. Secondly, while in the first 4 studies of this thesis the same operator performed all measurements, in the last two chapters 3 operators were responsible for performing measurements in the different centers involved, which could lead to examiner-related variability. In order to limit such problem, all operators received proper training before initiation of the study. In addition, we showed in chapter 2 that operators that receive training in the same protocol of muscle US have excellent intra-rater correlation coefficient (ICC). Based on that background, we are confident that large inter-or intra-observer variability could not be an issue in this study, such that it would change the results. Thirdly, in the last part of the thesis, HGS was not available for all patients, leading to patients with reduced muscle mass but unavailable muscle strength, being removed from the analysis because it was not possible to determine the presence or absence of sarcopenia. Given the strength of findings, that missing data would probably have improved the results. 


\section{THE USE OF MUSCLE US IN OTHER CONDITIONS}

Quadriceps muscle US has been successfully applied in different clinical settings using different metrics. During ICU stay muscle US was used to monitor the presence of muscle wasting $(33,34)$. While in patients with coronary artery disease (CAD), it seemed as accurate as $\mathrm{CT}$ or MRI in measuring RF diameter (3). Similar findings were shown in patients with chronic obstructive pulmonary disease (COPD) regarding RF cross-sectional area (CSA) (4), and volume (9). In particular, mid-thigh thickness (i.e. thickness of the quadriceps femoris) best correlated with fat-free mass by DEXA in patients with COPD, and it was also more sensitive than DEXA in detecting muscle changes after exercise intervention (35).

Currently, there is an increasing effort to recognize US as a reference practical tool for the assessment of low muscularity in the diagnosis of sarcopenia. In the elderly, an US sarcopenic index (USI) based on metrics obtained by US of the quadriceps vastus lateralis muscle (i.e. the ratio between muscle fascicle length and muscle thickness) has been recently developed by applying the Z-score obtained from a younger population with excellent correlation with skeletal muscle index (SMI) obtained by DEXA (36). While another study derived muscle thickness cutoffs from a Japanese young population applying the EWGSOP recommendation of 2 standard deviations below the mean (37).

\section{TOOLS USED}

We assessed quadriceps muscle thickness using 3 different ultrasound systems (Philips hd7xe, Logiq and General electric) depending on center availability. Body composition was assessed using the Body Composition Monitor (BCM, Fresenius Medical Care) in chapter 7, and Somaton Definition Flash CT scanner in the validation study. Handgrip strength was measured by a portable digital dynamometer (KERN \& SOHN GmbH, Germany). In chapter 4, we assessed the nutritional status of patients using the "Malnutrition Inflammation Score" (MIS) (38). We opted for the MIS composite tool because it was specifically designed for patients on chronic hemodialysis.

All data on demographics and blood parameters were collected from patient's file.

\section{IMPLICATIONS FOR FUTURE PRACTICE}

The skeletal muscle is not only vital to mobility, posture, strength and balance (39), but it is also a key metabolic and homeostatic organ system (40). Most importantly, it plays a pivotal role in protein metabolism, being the source of amino acids when catabolism is present, thus preserving essential organs $(40,41)$. However, the presence of a chronic catabolic state may cause an important reduction in skeletal muscle mass and lean body mass, which has serious consequences, such as muscle weakness and impaired physical function, leading to disability and increased morbidity and mortality (42). As stated previously, muscle loss is a frequent finding in patients with chronic and acute renal diseases (16), a phenomenon that has been associated to nutritional problems, the presence of acute and chronic comorbidities, a sedentary lifestyle, and the effect of the kidney diseases and its treatments $(43,44)(17)$. Given its utmost importance, the assessment and monitoring of muscle status should be an 
important part of the comprehensive nutritional evaluation of patients. Currently available and recommended techniques have important limitations that hamper the proper evaluation and monitoring of skeletal muscle in the renal setting. Imaging techniques such as MRI, CT and DEXA, are considered the reference methods. While they could provide a precise quantification of muscle tissues, they are expensive and not available for routine patient assessment, expose patients to excessive amounts of radiation in the case of $\mathrm{CT}$, and can have measurements biased by overhydration in the case of DEXA. Bedside tools are usually preferred because they provide immediate information allowing for prompt identification of patients with muscle loss. However, available techniques such as anthropometrics and BIA/BIS have important limitations, both techniques can be biased by hydration status, in the case of anthropometrics reproducibility and repeatability are limited, and in the case of BIA/BIS, surrogates of muscle mass (FFM and LBM) are estimated based on population specific regression equations (in the case of BIA), or rely on theoretical assumptions regarding the conversion from bioelectrical signals to estimations of body water compartments (in the case of BIS).

Bearing those limitations in mind, the ideal tool to assess skeletal muscle status in renal patients should have the following characteristics: low cost, high availability, no specialized staff, be an imaging technique, high portability to be used at the bedside of patients, sensitivity, reproducibility, repeatability, and not influenced by fluid status. All these characteristics have been considered in the development of this thesis and the choice of US of a new bedside tool to be applied in renal patients for the assessment and monitoring of muscle mass. The impact that this thesis may have in future practice are:

- Inclusion of quadriceps muscle US as a reference method for the diagnosis of low muscle mass - Increased awareness and more patients being evaluated for muscle loss, since US is currently available in every clinical setting and there is no need to invest in expensive tools

- Nutritional and physical interventions could be easily monitored during HD sessions by assessing patients' quadriceps muscle status with US

\section{IMPLICATIONS FOR FUTURE RESEARCH}

Future studies should focus on determining reference values of quadriceps muscle thickness by using normative data from healthy subjects from different ethnic background and multiple age intervals. This would allow for a global application of quadriceps muscle US in different clinical settings, while reference values obtained from the studied patient population limit their applicability. In addition, quadriceps muscle US should also be applied in interventional studies to confirm its sensitivity and validity as a monitoring tool. Finally, other metrics of muscle quantity and quality could be extensively studied and developed. For example, RF CSA has also been studied in patients on HD, presenting good correlation with PEW as diagnosed by the ISRNM criteria (45), however a formal assessment regarding its use as monitoring feature is still lacking, as well as its sensitivity as a mortality predictor. In regard to the study if muscle quality by US, the assessment of muscle echogenicity could indicate fibrosis and fat infiltration, and studies relating muscle echogenicity to muscle strength should be performed, because although muscle mass is not directly related to muscle strength, muscle quality is (46). 


\section{CONCLUSIONS}

In conclusion, this thesis met its objective of studying the applicability, validity and prognostic capacity of US in patients with kidney disease as a bedside tool for the assessment o skeletal muscle status. The main findings of the thesis are outlined below:

- Quadriceps muscle US has good reproducibility and repeatability when operators undergo proper training before applying it

- Quadriceps muscle US measurements are not influenced by fluid status

- Quadriceps muscle US is accurate and precise in comparison to CT

- Patients with ESKD on HD have reduced quadriceps muscle thickness in comparison to controls

- Quadriceps muscle US is sensitive to detect low muscularity and also to detect muscle changes in short periods of time

- Low muscle mass as assessed by quadriceps muscle US independently correlates with increased mortality risk and outperforms anthropometrics and BIS

- When quadriceps muscle US was used in combination to handgrip strength in the diagnosis of sarcopenia, it independently correlated with increased mortality 


\section{REFERENCES}

1. Tillquist M, Kutsogiannis DJ, Wischmeyer PE, Kummerlen C, Leung R, Stollery D, et al. Bedside ultrasound is a practical and reliable measurement tool for assessing quadriceps muscle layer thickness. JPEN J Parenter Enteral Nutr. 2014;38(7):886-90.

2. Ema R, Wakahara T, Mogi Y, Miyamoto N, Komatsu T, Kanehisa H, et al. In vivo measurement of human rectus femoris architecture by ultrasonography: validity and applicability. Clin Physiol Funct Imaging. 2013;33(4):267-73.

3. Thomaes T, Thomis M, Onkelinx S, Coudyzer W, Cornelissen V, Vanhees L. Reliability and validity of the ultrasound technique to measure the rectus femoris muscle diameter in older CAD-patients. BMC Med Imaging. 2012;12:7.

4. Seymour JM, Ward K, Sidhu PS, Puthucheary Z, Steier J, Jolley CJ, et al. Ultrasound measurement of rectus femoris cross-sectional area and the relationship with quadriceps strength in COPD. Thorax. 2009;64(5):418-23.

5. Baldwin C, Paratz J, Bersten A. Diaphragm and peripheral muscle thickness on ultrasound: intra-rater reliability and variability of a methodology using non-standard recumbent positions. Respirology. 2011;16(7):1136-43.

6. Parry SM, El-Ansary D, Cartwright MS, Sarwal A, Berney S, Koopman R, et al. Ultrasonography in the intensive care setting can be used to detect changes in the quality and quantity of muscle and is related to muscle strength and function. J Crit Care. 2015;30(5):1151 e9-14.

7. Bemben MG. Use of diagnostic ultrasound for assessing muscle size. Journal of strength and conditioning research. 2002;16(1):103-8.

8. Reeves ND, Maganaris CN, Narici MV. Ultrasonographic assessment of human skeletal muscle size. European journal of applied physiology. 2004;91(1):116-8.

9. Arbeille P, Kerbeci P, Capri A, Dannaud C, Trappe SW, Trappe TA. Quantification of muscle volume by echography: comparison with MRI data on subjects in long-term bed rest. Ultrasound Med Biol. 2009;35(7):1092-7.

10. Souza VA, Oliveira D, Cupolilo EN, Miranda CS, Colugnati FAB, Mansur HN, et al. Rectus femoris muscle mass evaluation by ultrasound: facilitating sarcopenia diagnosis in pre-dialysis chronic kidney disease stages. Clinics (Sao Paulo, Brazil). 2018;73:e392.

11. Puthucheary ZA, Rawal J, McPhail M, Connolly B, Ratnayake G, Chan P, et al. Acute skeletal muscle wasting in critical illness. Jama. 2013;310(15):1591-600.

12. Sabatino A, Regolisti G, Bozzoli L, Fani F, Antoniotti R, Maggiore U, et al. Reliability of bedside ultrasound for measurement of quadriceps muscle thickness in critically ill patients with acute kidney injury. Clin Nutr. 2017;36(6):1710-5.

13. Segura-Ortí E, Gordon PL, Doyle JW, Johansen KL. Correlates of Physical Functioning and Performance Across the Spectrum of Kidney Function. Clin Nurs Res. 2017:1054773816689282. 
14. Mueller N, Murthy S, Tainter CR, Lee J, Riddell K, Fintelmann FJ, et al. Can Sarcopenia Quantified by Ultrasound of the Rectus Femoris Muscle Predict Adverse Outcome of Surgical Intensive Care Unit Patients as well as Frailty? A Prospective, Observational Cohort Study. Annals of surgery. 2016;264(6):1116-24.

15. Puthucheary Z, Rawal J, McPhail M, Connolly B, Ratnayake G, Chan P, et al. Acute Skeletal Muscle Wasting in Critical Illness. Jama. 2013;310(15).

16. Sabatino A, Regolisti G, Delsante M, Di Motta T, Cantarelli C, Pioli S, et al. Noninvasive evaluation of muscle mass by ultrasonography of quadriceps femoris muscle in End-Stage Renal Disease patients on hemodialysis. Clin Nutr. 2019;38(3):1232-9.

17. Foley RN, Wang C, Ishani A, Collins AJ, Murray AM. Kidney function and sarcopenia in the United States general population: NHANES III. Am J Nephrol. 2007;27(3):279-86.

18. Giglio J, Kamimura M, Lamarca F, Rodrigues J, Santin F, Avesani C. Association of Sarcopenia With Nutritional Parameters, Quality of Life, Hospitalization, and Mortality Rates of Elderly Patients on Hemodialysis. J Ren Nutr. 2018;28(3):197-207.

19. Sabatino A, Regolisti G, Karupaiah T, Sahathevan S, Sadu Singh BK, Khor BH, et al. Protein-energy wasting and nutritional supplementation in patients with end-stage renal disease on hemodialysis. Clin Nutr. 2017;36(3):663-71.

20. Carrero JJ, Chmielewski M, Axelsson J, Snaedal S, Heimburger O, Barany P, et al. Muscle atrophy, inflammation and clinical outcome in incident and prevalent dialysis patients. Clin Nutr. 2008;27(4):557-64.

21. Miyamoto T, Carrero JJ, Qureshi AR, Anderstam B, Heimburger O, Barany P, et al. Circulating follistatin in patients with chronic kidney disease: implications for muscle strength, bone mineral density, inflammation, and survival. Clin J Am Soc Nephrol. 2011;6(5):1001-8.

22. Beddhu S, Pappas LM, Ramkumar N, Samore M. Effects of body size and body composition on survival in hemodialysis patients. J Am Soc Nephrol. 2003;14(9):2366-72.

23. Noori N, Kopple JD, Kovesdy CP, Feroze U, Sim JJ, Murali SB, et al. Mid-arm muscle circumference and quality of life and survival in maintenance hemodialysis patients. Clin J Am Soc Nephrol. 2010;5(12):2258-68.

24. Streja E, Molnar MZ, Kovesdy CP, Bunnapradist S, Jing J, Nissenson AR, et al. Associations of pretransplant weight and muscle mass with mortality in renal transplant recipients. Clin J Am Soc Nephrol. 2011;6(6):1463-73.

25. Oterdoom LH, van Ree RM, de Vries AP, Gansevoort RT, Schouten JP, van Son WJ, et al. Urinary creatinine excretion reflecting muscle mass is a predictor of mortality and graft loss in renal transplant recipients. Transplantation. 2008;86(3):391-8.

26. Fukasawa $\mathrm{H}$, Kaneko M, Niwa $\mathrm{H}$, Matsuyama $\mathrm{T}$, Yasuda $\mathrm{H}$, Kumagai $\mathrm{H}$, et al. Lower Thigh Muscle Mass Is Associated With All-Cause and Cardiovascular Mortality in Elderly Hemodialysis Patients. European journal of clinical nutrition. 2017;71(1). 
27. Rosenberger J, Kissova V, Majernikova M, Straussova Z, Boldizsar J. Body composition monitor assessing malnutrition in the hemodialysis population independently predicts mortality. J Ren Nutr. 2014;24(3):172-6.

28. Marcelli D, Usvyat L, Kotanko P, Bayh I, Canaud B, Etter M, et al. Body composition and survival in dialysis patients: results from an international cohort study. Clin J Am Soc Nephrol. 2015;10(7):1192-200.

29. Hwang S, Lee J, Lee S, Kim J, Kim M, Song J. Risk of overhydration and low lean tissue index as measured using a body composition monitor in patients on hemodialysis: a systemic review and meta-analysis. Ren Fail. 2018;40(1):51-9.

30. Broers N, Canaud B, Dekker M, van der Sande F, Stuard S, Wabel P, et al. Three compartment bioimpedance spectroscopy in the nutritional assessment and the outcome of patients with advanced or end stage kidney disease: What have we learned so far? Hemodial Int. 2020;24(2):148-61.

31. Dekker M, Konings C, Canaud B, van der Sande F, Stuard S, Raimann J, et al. Interactions Between Malnutrition, Inflammation, and Fluid Overload and Their Associations With Survival in Prevalent Hemodialysis Patients. J Ren Nutr. 2018;28(6):435-44.

32. Cruz-Jentoft AJ, Baeyens JP, Bauer JM, Boirie Y, Cederholm T, Landi F, et al. Sarcopenia: European consensus on definition and diagnosis: Report of the European Working Group on Sarcopenia in Older People. Age Ageing. 2010;39(4):412-23.

33. Reid CL, Campbell IT, Little RA. Muscle wasting and energy balance in critical illness. Clin Nutr. 2004;23(2):273-80.

34. Segers J, Hermans G, Charususin N, Fivez T, Vanhorebeek I, Van den Berghe G, et al. Assessment of quadriceps muscle mass with ultrasound in critically ill patients: intra- and inter-observer agreement and sensitivity. Intensive Care Med. 2015;41(3):562-3.

35. Menon MK, Houchen L, Harrison S, Singh SJ, Morgan MD, Steiner MC. Ultrasound assessment of lower limb muscle mass in response to resistance training in COPD. Respiratory research. 2012;13:119.

36. Narici M, McPhee J, Conte M, Franchi M, Mitchell K, Tagliaferri S, et al. Age-related alterations in muscle architecture are a signature of sarcopenia: the ultrasound sarcopenia index. J Cachexia Sarcopenia Muscle. 2021.

37. Fukumoto Y, Ikezoe T, Taniguchi M, Yamada Y, Sawano S, Minani S, et al. Cut-off Values for Lower Limb Muscle Thickness to Detect Low Muscle Mass for Sarcopenia in Older Adults. Clin Interv Aging. 2021;16:1215-22.

38. Kalantar-Zadeh K, Kleiner M, Dunne E, Lee GH, Luft FC. A modified quantitative subjective global assessment of nutrition for dialysis patients. Nephrol Dial Transplant. 1999;14(7):1732-8.

39. Shiozu H, Higashijima M, Koga T. Association of sarcopenia with swallowing problems, related to nutrition and activities of daily living of elderly individuals. J Phys Ther Sci. 2015;27(2):393-6.

40. Argiles JM, Campos N, Lopez-Pedrosa JM, Rueda R, Rodriguez-Manas L. Skeletal Muscle Regulates Metabolism via Interorgan Crosstalk: Roles in Health and Disease. J Am Med Dir Assoc. 2016;17(9):789-96.

41. Wolfe RR. The underappreciated role of muscle in health and disease. Am J Clin Nutr. 2006;84(3):475-82. 
42. Demling RH. Nutrition, anabolism, and the wound healing process: an overview. Eplasty. 2009;9:e9.

43. Kim HY. Nutritional intervention for a patient with diabetic nephropathy. Clinical nutrition research. 2014;3(1):64-8.

44. Thompson DD. Aging and sarcopenia. J Musculoskelet Neuronal Interact. 2007;7(4):344-5.

45. Sahathevan S, Khor B, Singh B, Sabatino A, Fiaccadori E, Daud Z, et al. Association of Ultrasound-Derived Metrics of the Quadriceps Muscle with Protein Energy Wasting in Hemodialysis Patients: A Multicenter Cross-Sectional Study. Nutrients. 2020;12(11):3597.

46. Johansen K, Shubert T, Doyle J, Soher B, Sakkas G, Kent-Braun J. Muscle Atrophy in Patients Receiving Hemodialysis: Effects on Muscle Strength, Muscle Quality, and Physical Function. Kidney Int. 2003;63(1). 

I Summary 
Protein energy wasting (PEW) is a frequent finding in patients with acute and chronic kidney impairment. It is characterized by the loss of patients' protein stores and energy fuels, characteristic of patients with kidney disease. It can be present in all stages of chronic kidney disease (CKD) and acute kidney injury (AKI), however it becomes clinically evident in the most advanced stages of both conditions. A number of metabolic derangements commonly observed in severe CKD and AKI (e.g. metabolic acidosis, inflammation, oxidative stress, and impaired insulin/insulin-like growth factor axis function), acute and chronic comorbidities and the kidney replacement therapy (KRT) per se, may have a negative impact on nutritional status. These pathogenetic factors may lead to muscle wasting through the alteration of the balance between muscle protein anabolism and catabolism.

The diagnosis of evaluation of PEW is usually based on a comprehensive assessment including patient history, physical examination, evaluation of nutrient intakes, biochemical markers and screening methodologies that provide a global picture of patients' protein and energetic reserve, as well as their nutritional risk. An important part of this evaluation is the assessment of body composition, which refers typically to the quantification of adipose tissue and skeletal muscle mass, with the identification of reduced muscle mass being critical.

Nowadays, several methodologies are available for the estimation/measurement of muscle mass. Imaging techniques such as MRI, CT and DEXA, are considered reference methods. While they could provide a precise quantification of muscle tissues, they are not available for routine patient assessment, while have other limitations regarding patients' safety and/or measurement precision in the presence of fluid overload (in the case of DEXA). Bedside tools are usually preferred because they provide immediate information allowing for prompt identification of patients with muscle loss. However, available techniques such as anthropometrics and BIA/BIS have important limitations intrinsic to the methods characteristics that hamper their use in the renal setting.

The aim of this dissertation is to study the applicability, validity and prognostic capacity of quadriceps muscle ultrasound (US) in patients with acute and chronic kidney disease on hemodialysis. The US technique seems to have all the necessary characteristics to be used as a diagnostic tool for the assessment of low muscle mass: low cost, high availability, no specialized staff, it's an imaging technique, high portability to be used at the bedside of patients. There was a need to investigate its sensitivity, reproducibility, repeatability, and to see if measurements were influenced by fluid status. All these characteristics have been considered in the development of this thesis and the choice of US as a new bedside tool to be applied in patients with kidney impairments for the assessment and monitoring of muscle mass.

In chapter 2, we studied the reliability of quadriceps muscle US. In this study, quadriceps RF and VI thickness of critically ill patients with AKI stage 3 on KRT were measured by two assessors. Intra and inter-observer reliability was evaluated using intraclass correlation coefficient (ICC). Results showed that the reproducibility of measurements (comparison 
between two assessors) was excellent, with an ICC value of 0.92 (across-site range $0.88-0.93$ ). The repeatability of the method (comparison of two measurements within the same assessor) was also very high, with an ICC value of 0.99 (across-site range $0.98-0.99$ ) for assessor 1 and an ICC value of 1.00 (across-site range $0.97-0.99$ ) for assessor 2 . No difference was found in measurements obtained before and after dialysis (11.5 (4.2) vs 11.4 (4.1) $\mathrm{mm}, \mathrm{P}=0.2498)$, independently from acute body weight changes due to fluid removal. Results from this study showed the quadriceps muscle US is reliable, with good repeatability and reproducibility.

In Chapter 3, we compared US with CT, a gold standard technique. Quadriceps RF and VI thickness of patients were blindly assessed at the same leg sites by both US and CT scans to estimate average difference in thickness, agreement and precision of the US methodology in comparison to CT. Results showed that the observed differential bias (between +0.04 and +0.26 $\mathrm{cm}$ depending on the muscle site) and the proportional bias (between $82 \%$ and $98 \%$ of the reference values, depending on the muscle site) were not statistically significant. A minimal loss in precision was found with US, but it was not significant.

In Chapter 4, quadriceps muscle US was applied in patients with ESKD on HD for the first time in the literature. After stratifying ESKD patients into subgroups based on nutritional variable cutoffs commonly used to define PEW in this clinical setting (BMI [ $\geq 23$ versus $<23 \mathrm{Kg} / \mathrm{m} 2$ ], albumin $[\geq 3.8$ versus $<3.8 \mathrm{~g} / \mathrm{dL}]$ ) and malnutrition inflammation score (MIS) status (<6 versus $\geq 6$ ), RF and VI thickness of patients with worse nutritional status defined by BMI and MIS were significantly lower than those of well-nourished ESKD-HD patients ( $P$ value range: $<0.001-<0.05$ ). In addition, we once again tested whether quadriceps muscle US measurements were affected by the HD procedure, with no differences between measurements performed before and after the dialysis session. In addition, we showed moderate correlations between quadriceps muscle US and other parameters of nutritional status such as BMI, MIS, mid-arm muscle area (MAMA) and a low correlation with serum albumin.

In Chapter 5, we report the data of a small study, in which we applied quadriceps RF and VI thickness by US in critically ill patients with AKI at baseline (within $72 \mathrm{~h}$ of hospitalization) and after 5 days to assess whether US was sensitive enough to detect changes in quadriceps muscle thickness during short periods of time. In that study, muscle thickness decreased by $15 \% \pm$ $13 \%$ within the first 5 days of ICU stay ( $P<0.001$ for all sites as compared to ICU admission). At baseline muscle thickness of patients was similar to that of healthy controls, however, after 5 days of hospitalization, patients had lower muscle thickness in comparison to controls. In addition, we identified that patients with more severe muscle loss had a higher probability of prolonged hospitalization, a finding that must be confirmed and further developed in future studies with a bigger sample size.

In Chapter 6, we normalized US and anthropometric (MAMA and MAMC) measurements of 181 patients on HD by height ${ }^{2}$ (indexed values) and derived different cut-offs of indexed RF and VI thickness by US, as well as indexed MAMA and MAMC based on the distribution (percentiles) 
of measurements in our sample of patients with ESKD on HD and studied the mortality risk predictability of 3 different cut-offs (p10, p25 and p50). Unlike indexed MAMC and MAMA, both proximal as well as distal indexed RF and $\mathrm{VI}$ thickness were significantly lower in patients who died as compared to patients who were alive at the end of the follow up period. The AUC values for the prediction of mortality were statistically significant at p25 and p50 for US derived measurements, but not for anthropometry. In the adjusted Cox-regression analysis, the indexed MAMC and indexed distal RF and VI thickness were independently related to mortality at p25 and p50, with the strongest association found when studying the indexed distal VI thickness at p50 (HR 2.16, 95\% Cl $1.27-3.69, \mathrm{P}=0.005)$.

In Chapter 7, we applied the cut-offs of indexed distal VI derived from the study described in chapter 6 in combination with HGS to diagnose sarcopenia and compared its prognostic capacity in comparison to sarcopenia as diagnosed by LTI combined with HGS. The applied cut-off for muscle US corresponded to $<3.44 \mathrm{~mm} / \mathrm{m}^{2}$ for males and $<3.52 \mathrm{~mm} / \mathrm{m}^{2}$ for females, while the cut-off used for LTI was obtained from an age-matched healthy control group with LTI $<10$ th percentile. The cut-off values for low HGS were $<27 \mathrm{~kg}$ for males and $<16 \mathrm{~kg}$ for females, as recommended by the revised EWGSOP 2. In this study, the correlation between LTI and QVIT was moderate $(r=0.37 ; p<0.001)$. When assessing the association with mortality, the non-adjusted analysis showed that low LTI had no association with mortality (HR 1.16 [95\% $\mathrm{Cl}$ 0.60-2.25], while low indexed VI thickness (HR 2.18 [95\% Cl 1.12-4.27]) and low HGS (HR 9.08 [95\% confidence interval ( $\mathrm{Cl}$ ) 2.12-38.8]) showed to increase the mortality risk. In the fully adjusted model, only the combination of low HGS and low indexed VIT thickness was significantly associated to higher mortality risk [HR 3.21 [95\% Cl 1.37-7.53].

The results of this thesis could generate several implications for the clinical practice in the renal setting. First, US for the assessment of quadriceps skeletal muscle could be included as a reference method for the bedside evaluation and diagnosis of low muscle mass. Second, it could lead to increased awareness and more patients being evaluated for muscle loss, since US is currently available in every ICU and dialysis center, results are immediate and there is no need to invest in expensive tools. Finally, nutritional and physical interventions could be easily monitored during HD sessions by assessing patients' quadriceps muscle status with US. The lack of reference values from a normative population, could drive future research in this field. 


\section{VALORIZATION}

The acute or chronic loss of kidney function is characterized by progressive worsening of nutritional status, in particular by an important reduction in protein stores and energy fuels, characteristic of the Protein-Energy Wasting (PEW) syndrome, a pathological condition associated with poor outcome. Thus, the assessment of nutritional status is essential both for preventing PEW in patients at risk, and for monitoring the success of nutritional and physical interventions in those with established PEW. In this regard, the assessment of skeletal muscle mass is of great importance, since many metabolic consequences of the loss of kidney function directly or indirectly promote muscle catabolism. Skeletal muscle is the largest store of Lean Body Mass (LBM) protein, and its quantitative/qualitative alterations may impact negatively on patients' quality of life, ability to perform activities of daily living and prognosis. Skeletal muscle can be estimated by conventional bedside techniques, such as anthropometry, bioimpedance spectroscopy (BIS), or bioimpedance analysis (BIA), which however, in the renal setting have important limitations already discussed throughout this thesis. While gold standard imaging techniques such as computerized tomography (CT), magnetic resonance imaging (MRI), and Dual Energy X-ray Absorptiometry (DEXA) are expensive, not available at the bedside, require highly specialized personnel and finally may imply exposure to radiation in the case of CT and DEXA.

In the present thesis quadriceps muscle ultrasound (US) was shown to be a reliable, accurate, portable and easy to perform tool to be applied at the bedside of patients with acute or chronic kidney disease on hemodialysis. It outperformed bedside techniques such as anthropometry and BIS in predicting mortality, and diagnosing sarcopenia when assessed alongside handgrip strength (HGS). Considering the relative low cost of modern US devices and its wide availability in every hospital ward, ICU and dialysis centers, US is not only a reliable tool to be used in the evaluation of quadriceps muscle, but it is also economically viable, since no additional costs are expected to start using US to evaluate skeletal muscle. Another advantage of this technique, is that no specialized staff is needed, with proper training renal dietitians could use this tool as part of their routine assessment of patients, not increasing the work load of nurses and nephrologists. In fact, considering that $50 \%$ of the studied population had lower quadriceps muscle thickness index and were at a higher risk of mortality for that, we suggest that muscle US indeed be a part of the routine evaluation of patients, being useful as a screening tool to identify patients with worse prognosis.

As expected for every other nutritional marker, the discriminative value of US when applied by itself is low, suggesting that it should not be used as sole prognostic tool to make clinical decisions at individual level in AKI and ESKD patients on HD. On this matter, a general nutritional status evaluation is a comprehensive assessment that includes not only body composition, but also patients' history, report of unintentional weight loss, functional parameters, physical examination, and other clinical and social parameters. On this ground, we suggest that quadriceps muscle US could add important information to multidimensional predictive 
models in renal patients. In fact, the assessment of skeletal muscle mass achieves its full potential in combination with other parameters, as shown in Chapter 7 . In addition, a diagnosis of low muscle mass or sarcopenia should be part of a more "holistic" approach, combining them with an evaluation of potentially modifiable factors such as dietary intake and physical activity. Findings should also be interpreted in view of its relation with a low health related quality of life, since these factors are often amenable to therapeutic intervention. Finally, nonnutritional factors associated with loss of muscle mass such as inflammation and metabolic acidosis should be assessed and corrected prior to nutritional and physical interventions, as they may hamper muscle anabolism. For instance, a patient with reduced quadriceps muscle thickness index identified at a screening phase and reduced muscle strength with adequate nutritional intake and absence of inflammation or metabolic acidosis, but with low physical activity may primarily benefit from a physical intervention. On the other hand, an inflamed and acidotic patient, with poor appetite and low nutrient intake, will primarily benefit from interventions that correct metabolic acidosis, a search into the cause of inflammation and targeted nutritional intervention. A physical rehabilitation program may be ineffective and much more difficult for this patient. With all those considerations in mind, we propose an "holistic" assessment that should be the basis for a personalized approach (Figure 1).

\begin{tabular}{|c|c|c|}
\hline $\begin{array}{l}\text { Screening phase: } \\
\text { - Muscle status by US } \\
\text { - Muscle strength by } \\
\text { HGS } \\
\text { - Functional } \\
\text { assessments } \\
\text { - Body weight history }\end{array}$ & $\begin{array}{l}\text { Identifying the } \\
\text { causes: } \\
\text { - Biochemical } \\
\text { parameters to } \\
\text { assess inflamamtion } \\
\text { and metabolic } \\
\text { acidosis } \\
\text { - Appetite and dietary } \\
\text { intake } \\
\text { - Physical activity } \\
\text { - Social and economic } \\
\text { conditions }\end{array}$ & $\begin{array}{l}\text { Defining interventions: } \\
\text { Individualized approach based } \\
\text { on the causes of low muscle } \\
\text { mass/stregth/performance: } \\
\text { - Nutritional } \\
\text { counseling/supplementation } \\
\text { - Physical rehabilitation } \\
\text { - Correction of metabolic } \\
\text { acidosis } \\
\text { - Identifying causes and } \\
\text { treating inflammation } \\
\text { - Nursing home/dental } \\
\text { problems }\end{array}$ \\
\hline
\end{tabular}




\section{PUBLICATIONS}

1. Sabatino A, Regolisti G, Benigno G, Di Mario F, Avesani CM, Fiaccadori E. Low skeletal muscle mass by computerized tomography is associated with increased mortality risk in end-stage kidney disease patients on hemodialysis. J Nephrol 2021; Oct 7. doi: 10.1007/ s40620-021-01167-y. Online ahead of print.

2. Sabatino A, Broers NJH, van der Sande FM, Hemmelder MH, Fiaccadori E, Kooman JP. Estimation of Muscle Mass in the Integrated Assessment of Patients on Hemodialysis. Front Nutr. 2021; 8: 697523.

3. Sabatino A, Maggiore U, Regolisti G, Rossi GM, Di Mario F, Gentile M, Farina MT, Fiaccadori E. Ultrasound for Non-invasive Assessment and Monitoring of Quadriceps Muscle Thickness in Critically III Patients with Acute Kidney Injury. Front Nutr 2021; 8:622823.

4. Avesani CM, Sabatino A, Guerra A, Rodrigues J, Carrero JJ, Rossi GM, Garibotto G, Stenvinkel P, Fiaccadori E, Lindholm B. A Comparative Analysis of Nutritional Assessment Using Global Leadership Initiative on Malnutrition Versus Subjective Global Assessment and Malnutrition Inflammation Score in Maintenance Hemodialysis Patients. J Ren Nutr 2021; 27: S1051-2276(21)00172-2.

5. Fiaccadori E, Sabatino A, Barazzoni R, Carrero JJ, Cupisti A, De Waele E, Jonckheer J, Singer $\mathrm{P}$, Cuerda $\mathrm{C}$. ESPEN guideline on clinical nutrition in hospitalized patients with acute or chronic kidney disease. Clin Nutr. 2021; 40:1644-1668

6. Cupisti A, Bolasco P, D'Alessandro C, Giannese D, Sabatino A, Fiaccadori E. Protection of Residual Renal Function and Nutritional Treatment: First Step Strategy for Reduction of Uremic Toxins in End-Stage Kidney Disease Patients. Toxins (Basel) 2021; 13: 289

7. Sabatino A, Cuppari L, Stenvinkel P, Lindholm B, Avesani CM. Sarcopenia in chronic kidney disease: what have we learned so far? J Nephrol. 2020 Sep 2. doi: 10.1007/s40620-020-00840-y.

8. Sahathevan S, Khor BH, Singh BKS, Sabatino A, Fiaccadori E, Daud ZAM, Ali MS, Narayanan SS, Tallman D, Chinna K, Goh BL, Gafor AHA, Ahmad G, Morad Z, Khosla P, Karupaiah T, On Behalf of the Patch Study Malaysia Investigators. Association of Ultrasound-Derived Metrics of the Quadriceps Muscle with Protein Energy Wasting in Hemodialysis Patients: A Multicenter Cross-Sectional Study. Nutrients. 2020; 12: 3597

9. Sabatino A, D’Alessandro C, Regolisti G, Di Mario F, Guglielmi G, Bazzocchi A, Fiaccadori E. Muscle mass assessment in renal disease: the role of imaging techniques. Quant Imaging Med Surg. 2020; 10: 1672-1686. 
10. Regolisti G, Sabatino A, Fiaccadori E. Exercise in patients on chronic hemodialysis: current evidence, knowledge gaps and future perspectives. Curr Opin Clin Nutr Metab Care. 2020; 23: 181-189.

11. Battaglia Y, Ullo I, Massarenti S, Esposito P, Prencipe M, Ciancio G, Provenzano M, Fiorini F, Andreucci M, Storari A, Sabatino A, Fiaccadori E, Granata A. Ultrasonography of Quadriceps Femoris Muscle and Subcutaneous Fat Tissue and Body Composition by BIVA in Chronic Dialysis Patients. Nutrients. 2020; 12: 1388.

12. Fiaccadori E, Cosola C, Sabatino A. Targeting the Gut for Early Diagnosis, Prevention, and Cure of Diabetic Kidney Disease: Is the Phenyl Sulfate Story Another Step Forward? Am J Kidney Dis. 2020; 75: 144-147

13. Sabatino A, Regolisti G, di Mario F, et al. Validation by CT scan of quadriceps muscle thickness measurement by ultrasound in acute kidney injury [published online ahead of print, 2019 Nov 15]. J Nephrol. 2019;10.1007/s40620-019-00659-2. doi:10.1007/s40620-019-00659-2

14. Gandolfini I, Regolisti G, Bazzocchi A, Maggiore U, Palmisano A, Piotti G, Fiaccadori E, Sabatino A. Frailty and Sarcopenia in Older Patients Receiving Kidney Transplantation. Front Nutr 2019; 6: 169

15. Hellerman M, Sabatino A, Theilla M, Kagan I, Fiaccadori E, Singer P. Carbohydrate and Lipid Prescription, Administration, and Oxidation in Critically Ill Patients with Acute Kidney Injury: A Post Hoc Analysis. J Ren Nutr. 2019; 29: 289-294

16. Cosola C, Rocchetti MT, Sabatino A, Fiaccadori E, Di lorio BR, Gesualdo L. Microbiota issue in CKD: how promising are gut-targeted approaches? J Nephrol. 2019; 32: 27-37

17. Cosola C, Sabatino A, di Bari I, Fiaccadori E, Gesualdo L. Nutrients, Nutraceuticals, and Xenobiotics Affecting Renal Health. Nutrients. 2018; 10(7)

18. Sabatino A, Piotti G, Cosola C, Gandolfini I, Kooman JP, Fiaccadori E. Dietary protein and nutritional supplements in conventional hemodialysis. Semin Dial. 2018; 31: 583-591

19. Sabatino A, Regolisti G, Delsante M, Di Motta T, Cantarelli C, Pioli S, Grassi G, Batini V, Gregorini M, Fiaccadori E. Noninvasive evaluation of muscle mass by ultrasonography of quadriceps femoris muscle in End-Stage Renal Disease patients on hemodialysis. Clin Nutr. 2019; 38: 1232-1239

20. Regolisti G, Maggiore U, Sabatino A, Gandolfini I, Pioli S, Torino C, Aucella F, Cupisti A, Pistolesi V, Capitanini A, Caloro G, Gregorini M, Battaglia Y, Mandreoli M, Dani L, Mosconi G, Bellizzi V, Di lorio BR, Conti P, Fiaccadori E; Gruppo di Studio "Esercizio fisico nel paziente con insufficienza renale cronica" of the Società Italiana di Nefrologia. Interaction of 
healthcare staff's attitude with barriers to physical activity in hemodialysis patients: A quantitative assessment. PLoS One. 2018; 13: e0196313.

21. Khor BH, Narayanan SS, Sahathevan S, Gafor AHA, Daud ZAM, Khosla P, Sabatino A, Fiaccadori E, Chinna K, Karupaiah T. Efficacy of Nutritional Interventions on Inflammatory Markers in Haemodialysis Patients: A Systematic Review and Limited Meta-Analysis. Nutrients. 2018 Mar 23;10(4)

22. Sabatino A, Theilla M, Hellerman M, Singer P, Maggiore U, Barbagallo M, Regolisti G, Fiaccadori E. Energy and protein in critically ill patients with AKI: A prospective, multicenter observational study using indirect calorimetry and protein catabolic rate. Nutrients. 2017; 9(8).

23. Sabatino A, Regolisti G, Gandolfini I, Delsante M, Fani F, Gregorini MC, Fiaccadori E. Diet and enteral nutrition in patients with chronic kidney disease not on dialysis: a review focusing on fat, fiber and protein intake. J Nephrol. 2017; 30:743-754

24. Sabatino A, Regolisti G, Cosola C, Gesualdo L, Fiaccadori E. Intestinal microbiota in type 2 diabetes and chronic kidney disease. Curr Diab Rep. 2017; 17: 16

25. Sabatino A, Regolisti G, Karupaiah T, Sahathevan S, Sadu Singh BK, Khor BH, Salhab N, Karavetian M, Cupisti A, Fiaccadori E. Protein energy wasting and nutritional supplementation in patients with end-stage renal disease on hemodialysis. Clin Nutr 2017; 36: 663-671

26. Sabatino A, Regolisti G, Bozzoli L, Fani F, Antoniotti R, Maggiore U, Fiaccadori E. Reliability of bedside ultrasound for measurement of quadriceps muscle thickness in critically ill patients with acute kidney injury. Clin Nutr. 2017;36:1710-1715

27. Fiaccadori E, Sabatino A, Morabito S, Bozzoli L, Donadio C, Maggiore U, Regolisti G. Hyper/ Hypoglicemia and acute kidney injury in critically ill patients. Clin Nutr 2016; 35: 317-321.

28. Sabatino A, Regolisti G, Brusasco I, Cabassi A, Morabito S, Fiaccadori E. Alterations of intestinal barrier and microbiota in chronic kidney disease. Nephrol Dial Transplant 2015; 30: 924-933

29. Fiaccadori E, Sabatino A, Schito F, Angella F, Malagoli C, Tucci M, Cupisti A, Capitanini A, Regolisti G. Barriers to physical activity in chronic hemodialysis patients: A single-center pilot studyin an italian dialysis facility. Kidney Blood Press Res 2014; 39: 169-175.

30. Sabatino A, Regolisti G, Antonucci E, Cabassi A, Morabito S, Fiaccadori E. Intradialytic Parenteral Nutrition in End-Stage Renal Disease: practical aspects, indications and limits. J Nephrol 2014; 27: 377-383.

31. Sabatino A, Regolisti G, Maggiore U, Fiaccadori E. Protein/energy debt in critically ill children in the pediatric intensive care unit: Acute Kidney Injury as a major risk factor. J Ren Nutr 2014; 24: 209-218. 


\section{ACKNOWLEDGEMENT}

In this long journey many were the people that directly or indirectly helped me to arrive here. First of all, I would like to thank Maastricht University and the School of NUTRIM for giving me the opportunity to be an external PhD student. It allowed me to achieve my dream without having to let other dreams go.

Next, my deepest gratitude and appreciation for my supervisor, Prof Jeroen Kooman, always available when needed. Thank you for your valuable feedbacks on my work, advice and guidance.

To my work family in Parma, especially my co-supervisor Prof Enrico Fiaccadori, that believed in me more than eight years ago, when I was new in Italy and in the academia. You helped me grow so much in those years that I was able to become this independent researcher that I am today. Thank you also to Prof Giuseppe Regolisti and Prof Umberto Maggiore, my statistical mentors.

To my family, my husband Marcelo, and my children Luca and Lorenzo. Thank you for your patience, support and love.

Alice 
152 


\section{CURRICULUM VITAE}

Alice Baganha Sabatino was born on January $24^{\text {th }}, 1986$, in Brazil. She is an Italian-Brazilian dietitian and earned her bachelor degree in "Nutrition and Metabolism" from the University of São Paulo, Brazil, in 2008, her first level masters in Nutrition and Dietetics in Nephrology from University of Pisa, Italy in 2013, and her masters of science in Food and Human Nutrition Science from University of Milan, Italy, in 2016. She is currently a PhD candidate in the school of "Nutrition and Translational Research in Metabolism" in Maastricht University, Netherlands. She conducted her research at Parma University Hospital, in Italy, where she currently works as renal dietitian and researcher.

Mrs Sabatino is a licensed nutritionist and dietitian by the Ministry of Public Health in Brazil since 2008, and in Italy since 2015. She has been working as a clinical dietitian since 2009, starting in Brazil and then transferring to Italy in 2015. Before obtaining the Italian professional license to work as a dietitian, she started working as research assistant at the Nephrology department of Parma University Hospital in 2013. In 2015, Alice won a young investigator research grant by the Italian Society of Parenteral and Enteral Nutrition (SINPE), which helped funding this thesis. She is currently very much engaged in research, being part of the board of the European Renal Nutrition working group of the European Renal Association (ERA), author of the "Nutrition Support in chronic hemodialysis" module of the Life Long Learning (LLL) program in Clinical Nutrition and Metabolism of the European Society for Clinical Nutrition and Metabolism (ESPEN), and is a member of the working group for the new ESPEN guideline on clinical nutrition in hospitalized patients with acute or chronic kidney disease. 
